\title{
Essays on Horizontal Merger Analysis
}

\author{
by
}

Gang Li

A thesis submitted to the Faculty of Graduate and Postdoctoral Affairs in partial fulfillment of the requirements for the degree of

Doctor of Philosophy

in

Economics

Carleton University

Ottawa, Ontario

(C) 2013, Gang Li 


\section{Abstract}

This dissertation consists of three essays that study the economic impact of horizontal mergers. Chapters 1 and 2 address the merger paradox about merger profitability. Briefly put, we examine two oligopoly models of both price and quantity competition and demonstrate that mergers are profitable under certain conditions. To be more specific, we analyze in Chapter 1 the effects of mergers when every firm in the market faces a capacity constraint. We show that a merger has no effect on equilibrium prices and profits if pure-strategy equilibrium prevails both before and after the merger. Otherwise prices increase and the merger is profitable. Specifically if mixed-strategy equilibrium prevails both before and after the merger, the support of the price distribution will shift upward, and the post-merger price distribution of each firm will dominate its pre-merger price distribution.

We show in Chapter 2 that product differentiation can also resolve the merger paradox associated with quantity competition by broadening the range of parameters over which mergers are profitable. To be more specific, we demonstrate that a merger is profitable if the number of competitors is small, and if the substitutability is high between the insiders but low between each insider and the outsiders. The post-merger prices of the insiders are higher when the substitutability is higher between the insiders or lower between each insider and the outsiders.

Chapter 3 extends the model in Chapter 2 to study how efficiencies affect postmerger equilibrium. Specifically, we consider a situation where a merger reduces the marginal cost of the merging firms. We find that, depending on the degree of substitutability between the merging products, efficiencies can have opposite effects on 
post-merger prices. When the degree of substitutability is sufficiently high, efficiencies tend to reduce post-merger prices, as the conventional wisdom suggests. However, if the degree of substitutability is lower, efficiencies have the unconventional effect of raising the post-merger prices. In this case, prices rise with efficiencies and might be eventually pushed up above the pre-merger level as efficiencies grow. Our analysis suggests that measures such as pass-through rate and the Upward Pricing Pressure test might lead to misleading conclusions regarding the price effects of a merger. 
To my parents,

Thank you for everything.

To my sister, Li Tao

We will never forget you. 


\section{Acknowledgements}

My first, and deepest appreciation must go to my advisor Professor Zhiqi Chen. It has been three years since we first sat down and discussed the topics of this thesis. It is his every effort of guidance and advice that makes this thesis possible. My acknowledgement goes well beyond the academic help, and the most valuable financial and professional supports from him shall never be forgotten. I am in every sense indebted to Zhiqi.

I also owe a huge debt of gratitude to my committee members Professor Gamal Atallah and Professor Aggey Semenov. The extensive advice gave me great illumination. Furthermore, Gamal's painstaking proofreading and Aggey's wholehearted encouragements are truly appreciated.

I shall give special thanks to my examiners Professor Andrew Eckert and Professor Saul Schwartz for their very valuable advice and comments.

I am so grateful to Marge. Her diligent work is always a true support to all students in the department.

Last but not least, I shall give deepest thank to my wife, my parents, my sister and her family, and my son. Their support is most precious in the whole world. 


\section{Table of Contents}

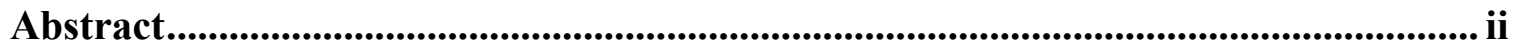

Acknowledgements .................................................................................................................. v

Table of Contents ........................................................................................................ vi

List of Tables .............................................................................................................................. viii

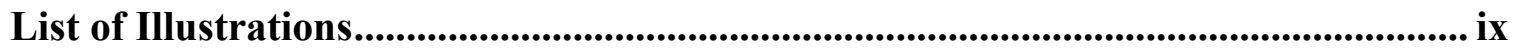

List of Appendices...................................................................................................................... $\mathrm{x}$

Chapter 1: Horizontal Mergers in the Presence of Capacity Constraints ................................1

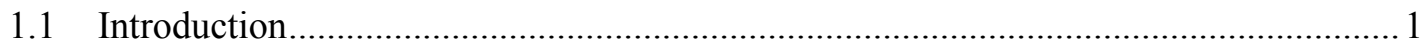

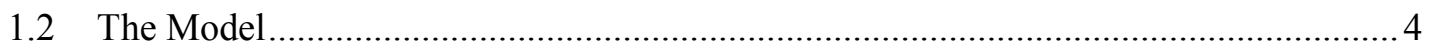

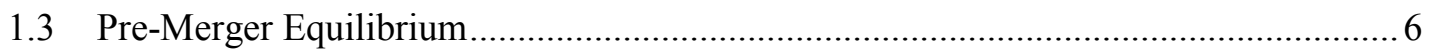

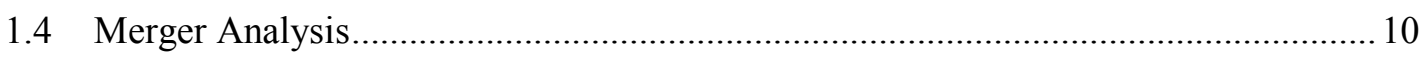

1.5 A Model with Linear Demand Function.....................................................................2

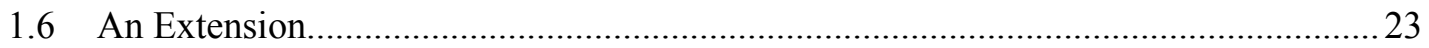

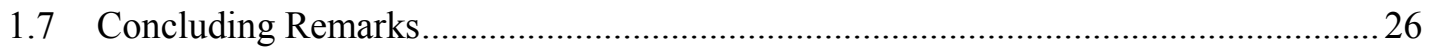

Chapter 2: Horizontal Merger in a Cournot Model with Differentiated Products..............28

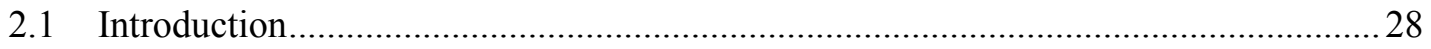

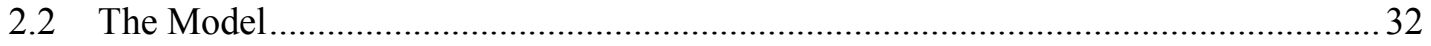

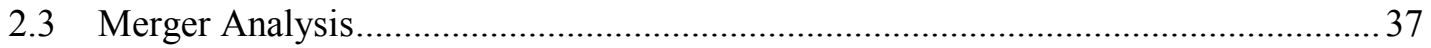

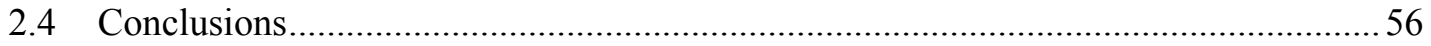

Chapter 3: Merger Efficiencies in a Cournot Model with Differentiated Products ............58

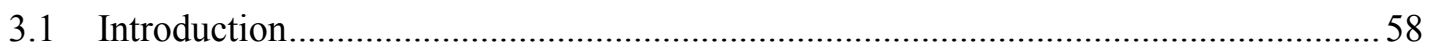

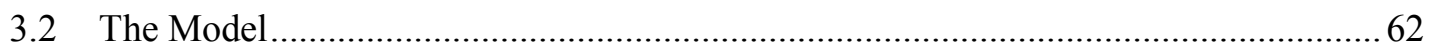

3.3 An Application: UPP Test …………………………………………………..... 79 


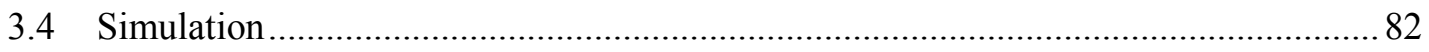

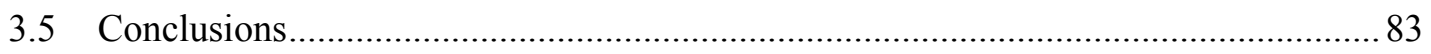

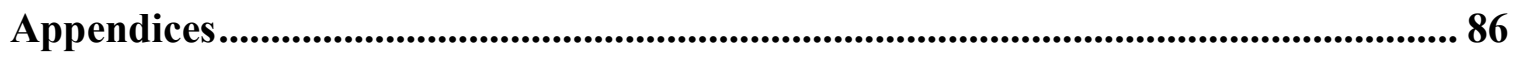

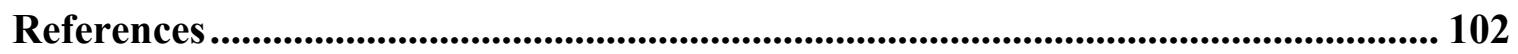




\section{List of Tables}

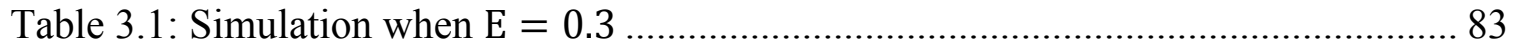

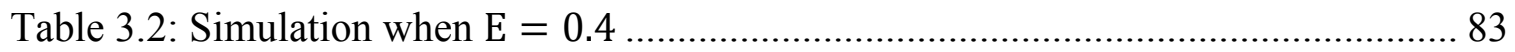




\section{List of Illustrations}

Figure 1.1 Equilibrium Strategies Before and After the Merger ................................ 16

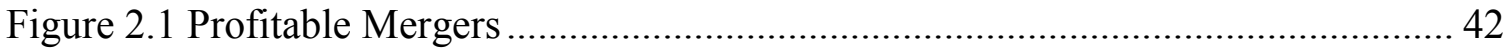

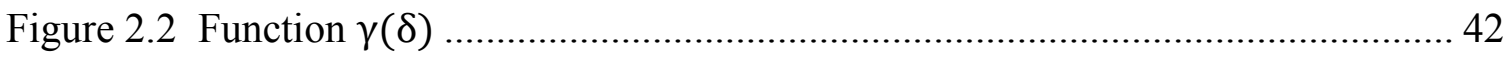

Figure 2.3 A Sufficient Condition for Profitable Mergers ............................................. 43

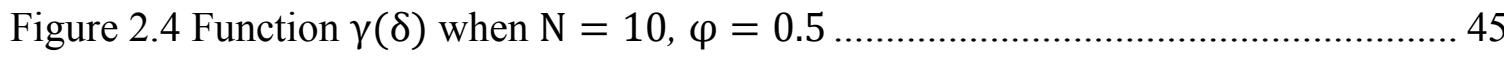

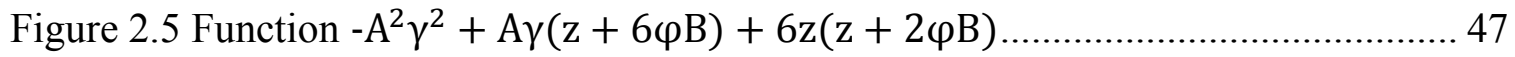

Figure 2.6 How N Affects Merger Profitability .......................................................... 49

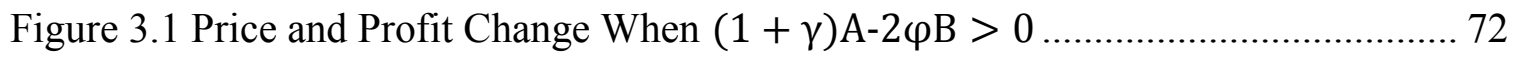

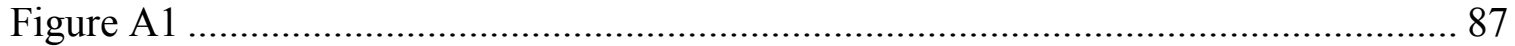

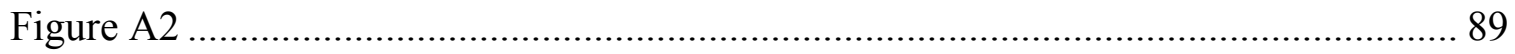

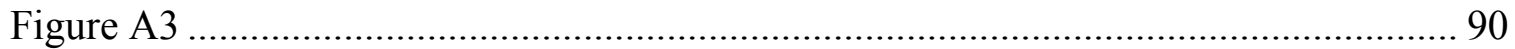




\section{List of Appendices}

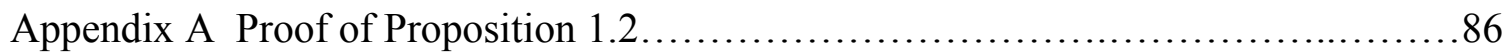

Appendix B Post-Merger Mixed Strategy Equilibriums in the Model of Section1.5..... 91

Appendix C Post-Merger Mixed Strategy Equilibriums in the Model of Section1.6.... 96

Appendix D Merger Simulation............................................99 


\section{Chapter 1: Horizontal Mergers in the Presence of Capacity Constraints}

\subsection{Introduction}

A widely held belief among economists is that horizontal mergers between oligopolistic firms raise prices. ${ }^{1}$ But a recent article by Froeb, et al. (2003) challenges this belief by claiming that that "[i]n the case where the merged firm is capacity-constrained, there is no merger price effect." They support their claim by numerical simulations of a merger model with price competition among capacity-constrained firms that operate parking lots. In the model, consumers maximize their utility by balancing between parking fees and transportation cost. They show that, although the capacity constraints on the non-merging firms drive up post-merger prices as has long been believed, the capacity constraints on the merging firms depress post-merger prices, and the latter effect is greater than the former. When both merging firms are capacity constrained, a merger has simply zero effect on price, quantity, consumer surplus and total welfare. Similar results are found in Higgins, et al. (2004), another computational work.

In contrast to the numerical simulations by Froeb, et al. (2003) and Higgins, et al. (2004), this chapter conducts a theoretical examination on the effects of horizontal mergers in the presence of capacity constraints using the familiar Bertrand-Edgeworth model. To be more specific, we study a model in which firms are identical before a merger of two firms. Firms produce a homogeneous product, and they set prices

\footnotetext{
${ }^{1}$ For theoretical demonstrations of this result, see, for example, Salant, et al. (1983), Deneckere and Davidson (1985), McAfee, et al. (1992), Perry and Porter (1985), Farrell and Shapiro (1990). Empirically, Weinberg (2008) surveys the literature on the price effects of horizontal mergers, and finds that prices increased after a merger in the majority of cases that have been examined.
} 
simultaneously taking into consideration their capacity constraints. We show that the characteristics of the equilibrium and accordingly the effects of the merger depend on the capacity level of each firm. For a sufficiently low capacity level, a pure-strategy equilibrium prevails both before and after the merger. In this case, the merger has no effect on the equilibrium price, as in Froeb, et al. (2003). However, a higher capacity level - but not so high as to drive the equilibrium price down to the marginal cost - allows mixed-strategy equilibriums to emerge and the merger leads to higher prices. In particular, in the case where mixed-strategy equilibrium prevails both before and after the merger, the post-merger price distribution of each firm dominates its pre-merger price distribution and the support of the price distribution shifts upward after the merger. Consequently, a merger raises the profits of every firm unless a pure-strategy equilibrium prevails before and after the merger. ${ }^{2}$

This chapter is related to three strands of literature. First, of direct relevance are the analyses on mergers under Bertrand competition. It has been shown that when firms produce differentiated products and compete in prices, a merger raises price and is more profitable for the merging firms (Deneckere and Davidson 1985, Braid 1986 and 1999, Reitzes and Levy 1990, and Levy and Reitzes 1992). While several numerical simulations of mergers have incorporated capacity constraints into price competition models (Froeb, et al. 2003, Higgins et al. 2004, Choné and Linnemer 2010), there has been very little formal theoretical analysis of mergers in the presence of capacity

\footnotetext{
${ }^{2}$ Apart from the parking industry studied by Froeb, et al. (2003), other examples of industries where firms face capacity constraints include airline and hotel industry. Empirically Gillen and Martin (2009) study airline price volatility and conclude that price volatility is endogenous and might be a part of carrier pricing strategy. This pricing volatility might resemble the mixed strategy equilibrium in our model.
} 
constraints. The only exception is a brief analysis in Hirata (2009), which points out the possibility that a merger could be unprofitable when firms face capacity constraints.

Second, also of some relevance are the theories of mergers in the case of Cournot competition. It is now well known that in the absence of efficiency gains, mergers under Cournot competition are often unprofitable (the merger paradox). ${ }^{3}$ A number of authors, including Perry and Porter (1985), McAfee and Williams (1992), and Daughety (1990), have proposed various ways to resolve the merger paradox. In particular, Perry and Porter (1985) show that mergers can be profitable under Cournot competition if firms face a rising marginal cost curve. This bears a relationship to this chapter in the sense that capacity constraints can be viewed as an extreme form of rising marginal cost.

Third and finally, our analysis relates to the literature on the Bertrand-Edgeworth model. Notable contributions to this literature include Dasgupta and Maskin (1986a and 1986b) that derive the existence conditions of mixed-strategy equilibrium. Kreps and Scheinkman (1983) and Osborne and Pitchik (1986) characterize the equilibriums for the duopoly case where firms are asymmetric. Allen and Hellwig (1986) explore the relation between perfectly competitive and monopolistically competitive equilibriums and characterize the mixed strategy equilibrium in a large oligopoly market using a BertrandEdgeworth model. They are followed by Vives (1986) who uses a different rationing rule. More recently, Hirata (2009) and Francesco and Salvadori (2009 and 2010) investigate the case of three (and N) asymmetric firms in more general settings. This sizable literature has made extensive achievement in characterizing both pure and mixed strategy

\footnotetext{
${ }^{3}$ See, for example, Salant, et al. (1983), and Lommerud and Sørgard (1997). Davidson and Mukherjee (2007) show that free entry would restore merger profitability but cost synergy is indispensible.
} 
equilibriums, in a small or large market, with symmetric or asymmetric capacity constraints. $^{4}$

This chapter is organized as follows. A basic model with three firms is presented in section 1.2, while the pre-merger equilibrium in this model is analyzed in section 1.3. In section 1.4 the effects of a merger are examined using the basic model, followed by the special case of linear demand in section 1.5. Section 1.6 investigates the robustness of our results in a general model with $n$ identical firms. While it is not possible to derive a complete characterization of the mixed strategy equilibriums in this general model, we are able to show that the effects of a merger remain qualitatively the same. Section 1.7 concludes.

\subsection{The Model}

In the basic model, there are three identical firms, $\mathrm{A}, \mathrm{B}$ and $\mathrm{C}$, that produce a homogeneous good. The marginal cost of production is normalized to zero and there is no fixed cost. Furthermore, each firm faces a capacity constraint $K$. In other words, a firm's output cannot exceed $K$. Let $p$ denote the price of the good. A firm's strategy set can be written as $\boldsymbol{A}_{i}=\left\{F_{i} \mid F_{i} \in[0,1], F_{i}=\int f_{i}(p) d p\right\}$ for $i=\{A, B, C\}$. Firms choose their strategies simultaneously.

Let $Q=D(p)$ denote the demand function for the good, and $P(Q)=D^{-1}(Q)$ denote the corresponding inverse demand function. Assume that $D(\cdot)$ is decreasing, concave

\footnotetext{
${ }^{4}$ Note that, for the most part, this strand of literature does not study mergers. The only exception is Hirata (2009), which contains a short section on a special example of merger paradox.
} 
and twice continuously differentiable. To make the merger analysis interesting, we assume that $2 K<D(0){ }^{5}$

As in the standard Bertrand model, consumers will want to buy from whichever firm that offers the lowest price. However, when the demand facing the firm with the lowest price exceeds its capacity, consumers have to be rationed. In this analysis we adopt the same rationing rule as that in Levitan and Shubik (1972), Kreps and Scheinkman (1983), Osborne and Pitchik (1986), Vives (1986), Hirata (2009) and Francesco and Salvadori (2009). Specifically, a firm offering a price $p_{i}$ faces the residual demand $D\left(p_{i}\right)-K_{j}$ if another firm $j$ offers a lower price $p_{j}$ but cannot satisfy $D\left(p_{j}\right)$ due to the capacity constraint. Note that this rationing rule leads to the same result as the efficient rationing rule under which output of the lowest-price producer goes first to consumers with the greatest evaluation of that product. $^{6}$

An important element in the analysis of the Bertrand-Edgeworth model is the Cournot best-response function. Define $q_{i}\left(\sum_{j \neq i} K_{j}\right)$ as firm $i$ 's Cournot best-response function when its rivals all produce at their capacities. In other words, $q_{i}\left(\sum_{j \neq i} K_{j}\right)$ is solved from firm $i$ 's first-order condition

$$
P\left(q_{i}+\sum K_{-i}\right)+q_{i} P^{\prime}\left(q_{i}+\sum K_{-i}\right)=0
$$

\footnotetext{
${ }^{5}$ If, instead, $2 K>D(0)$, the equilibrium price would be equal to marginal cost before a merger, because each firm would have the same incentive to undercut its rivals as in the standard Bertrand model without capacity constraints. But in this chapter we focus on situations where the pre-merger equilibrium is different from that of standard Bertrand model.

${ }^{6}$ Allen and Hellwig (1986), Dasgupta and Maskin (1986b) and Cheviakov and Hartwick (2005) use a different rationing rule $\frac{D\left(p_{i}\right)}{D\left(p_{j}\right)}\left[D\left(p_{j}\right)-K_{j}\right]$ (where $p_{i}>p_{j}$ ) under which the unsatisfied demand at lower prices goes to firms with higher prices at a discounted rate, for the reason that some of these unsatisfied demand simply left the market when consumers have to buy at higher prices. However the residual demand of a high price firm under our rationing rule is even smaller.
} 
Since all firms are identical, $q_{i}(\cdot)$ takes the same form for $i=A, B, C$. Thus, we can drop the subscript $i$ and write it as $q(\cdot)$.

The concavity of $D(\cdot)$ ensures the existence of a mixed-strategy equilibrium for $K$ in a certain range. ${ }^{7}$ To see this, note that mixed-strategy equilibrium requires firm $i^{\prime} \mathrm{s}$ capacity $K_{i}$ to be greater than its Cournot best-response quantity $q_{i}\left(\sum K_{-i}\right)($ Kreps and Sheinkman 1983). Totally differentiating (1.1) we obtain $\frac{d q_{i}}{d \sum K_{-i}}=\frac{d q_{i}}{d((n-1) K)}=$ $-\frac{P^{\prime}+q_{i} P^{\prime \prime}}{2 P^{\prime}+q_{i} P^{\prime \prime}}$, which is less than 0 if $P^{\prime \prime} \leq 0$ (or equivalently $D^{\prime \prime} \leq 0$ ). Then $q_{i}$ falls as $K$ increases, so that $K$ is bound to exceed $q_{i}$ at some point. Hence, $D^{\prime \prime} \leq 0$ guarantees the existence of a mixed-strategy equilibrium for some range of $K$.

Note that $D^{\prime \prime} \leq 0$ also ensures that $p D(\cdot)$ is strictly concave in $p$. The strict concavity of $p D(\cdot)$, in turn, implies that the cumulative distribution function $(\mathrm{CDF})$ of an equilibrium mixed strategy is strictly increasing in its support (Osborne and Pitchik 1986). Thus the support of mixed strategies is identical to the price range. In other words, we can rule out the possibility that some prices in the price range are not in the support of mixed strategies.

\section{$1.3 \quad$ Pre-Merger Equilibrium}

The level of production capacity $K$ is crucial in determining the outcome of price competition. Broadly speaking, very small capacities lead to a pure-strategy equilibrium,

\footnotetext{
${ }^{7}$ Note that concave demand is not necessary for the existence of mixed strategy equilibrium: it is a subset of demand that Dasgupta and Maskin (1986a, 1986b) assume. Allen and Hellwig (1986) further lift the limit on downward sloping demand. However, concave demand simplifies our analysis, as is claimed in the following paragraph. For formal proof of existence of mixed strategy equilibrium (in general models and in the Bertrand-Edgeworth model), see Dasgupta and Maskin (1986a and 1986b).
} 
and greater capacities give rise to mixed-strategy equilibrium. It is, therefore, necessary to pin down the threshold level of $K$ that separates the two types of equilibriums.

\subsubsection{The Threshold for a Mixed-Strategy Equilibrium}

In the literature, there are two different sufficient conditions for mixed-strategy equilibrium to take place. The first one, suggested by Kreps and Scheinkman (1983), requires that - in a duopoly game, for instance - either firm has a capacity greater than the Cournot best-response quantity when its rival produces at full capacity, i.e., $K_{i}>q_{i}\left(K_{j}\right)$ where $q_{i}\left(K_{j}\right)$ is firm $i$ 's Cournot best-response quantity. The second sufficient condition, due to Osborne and Pitchik (1986), states that mixed-strategy equilibrium occurs if the firm with a larger capacity has an incentive to raise its price above the competitive level $P\left(\sum K_{i}\right)$ whenever the smaller firm produces at full capacity. On the surface, these two conditions seem quite different, the former expressed in terms of quantity response while the latter is expressed in terms of price response. But both convey the idea that for mixed-strategy equilibrium to occur, one firm must have an incentive to cut production below its capacity (or equivalently raise price above the competitive level) whenever its rival produces at full capacity.

In this analysis we adopt the sufficient condition of Kreps and Sheinkman (1983).

Formally, we have the following result:

Lemma 1.1 There exists a unique $\widetilde{K}$ such that

$\widetilde{K}=\operatorname{argmax}_{q} q D^{-1}(q+2 \widetilde{K})$.

Furthermore, $K \leq \operatorname{argmax}_{q} q D^{-1}(q+2 K)$ if and only if $K \leq \widetilde{K}$. 
-Proof: The Cournot best response $q(2 K)$ is the solution to $\max _{q} q D^{-1}(q+2 K)$. Conducting comparative statics on the first-order condition associated with this optimization problem, we obtain

$$
\frac{d q}{d K}=-\frac{2 P^{\prime}+2 q P^{\prime \prime}}{2 P^{\prime}+q P^{\prime \prime}}<0 .
$$

Given the assumptions on the demand function, we know that $q(0)>0$ and $q(2 K)=0<2 K$ for $K$ sufficiently large. Thus, there is a unique $\widetilde{K}$ that satisfies (1.2). Since the function $K-q(2 K)$ is increasing in $K$, we have $K \leq \operatorname{argmax}_{q} q D^{-1}(q+2 K)$ if and only if $K \leq \widetilde{K}$.

Lemma 1.1 implies that the pre-merger equilibrium is one of pure strategies if each firm's capacity is small enough that $K \leq \widetilde{K}$. On the other hand, mixed strategy equilibrium occurs if $K>\widetilde{K}$.

\subsubsection{Pure-Strategy Equilibrium}

As indicated above, pure-strategy equilibrium occurs if $K \leq \widetilde{K}$ and that implies $K \leq q(2 K)$, i.e., the capacity of a firm must be no greater than its Cournot best-response quantity when both rivals produce at full capacity. In this situation, all three firms produce at full capacities and sell their product at equilibrium price $P(3 K)$. No firm has any incentive to cut price below $P(3 K)$, because it would not be able to produce any additional units to meet the increased demand. Nor does it want to raise its price above $P(3 K)$, because $K \leq \widetilde{K}$ implies that such a move would reduce its profit. 


\subsubsection{Mixed-Strategy Equilibrium}

A mixed-strategy equilibrium occurs if $K>\widetilde{K}$. Let $F_{i}$ denote an equilibrium mixed strategy of firm $i$, and $\left(p^{l}, p^{h}\right)$ the range of equilibrium prices. As shown in Osborne and Pitchik (1986), the concavity of $p D$ implies that the support of $F_{i}$ is identical to $\left(p^{l}, p^{h}\right)$.

Since all firms are identical before the merger, we focus on the symmetric equilibrium where all firms adopt the same mixed strategy, i.e., $F_{1}=F_{2}=F_{3} \equiv F$. The profit of firm $i$ when it chooses price $p_{i}$ and its rivals both play equilibrium strategies $F$ is

$$
\pi_{i}\left(p_{i}, F\right)=F^{2} p_{i} \cdot(D-2 K)+\left(1-F^{2}\right) p_{i} K
$$

From the existing studies of the Bertrand-Edgeworth model, we know that $F$ and $\pi_{i}$ have the following properties:

(1) $F$ is continuous everywhere. While a jump point may exist in the equilibrium mixed strategy of the larger firm when firms have different capacity levels, $F$ does not have a jump point in the case of identical firms (Kreps and Scheinkman 1983, Allen and Hellwig 1986, Dasgupta and Maskin 1986, Osborne and Pitchik 1986). Intuitively this is because if there is a jump point for one firm, it must be true for the other firm. But that cannot be an equilibrium as each firm would want to undercut the other one to obtain greater profit and thus avoid a positive probability at that point.

(2) The equilibrium profit of firm $i, \pi_{i}$, must be maximized and constant on the entire support of $F$. This implies that $\pi_{i}$ must be constant everywhere within the price range $\left(p^{l}, p^{h}\right)$.

(3) The support of $F$ has to be an open set. In other words, $p^{l}$ and $p^{h}$ can only be infinitely approached but never reached. 
Since the expected profit of a firm is everywhere maximized and constant, $\pi_{i}\left(p_{i}, F\right)=p^{l} K$. Using this and (1.3), we derive the equilibrium strategy $F=\sqrt{\frac{\left(p-p^{l}\right) K}{p(3 K-D)}}$. The supremum of the price support is determined by $p^{h}=\operatorname{argmax}_{p} p(D-2 K)$. This implies that $D\left(p^{h}\right)>2 K$ and $p^{h}<P(2 K)$. Thus, $\left(p^{l}, p^{h}\right)$ must be a subset of $(P(3 K), P(2 K))$. Moreover,

Lemma 1.2 An increase in $K$ reduces both $p^{l}$ and $p^{h}$.

-Proof: Noting that $p^{h}=\operatorname{argmax}_{p} p(D(p)-2 K)$, we totally differentiate the first-order condition to obtain: $\left(2 D^{\prime}+p^{h} D^{\prime \prime}\right) d p^{h}=2 d K$, from which we find $\frac{d p^{h}}{d K}<0$. Since $\pi_{i}=p^{h}\left(D^{h}-2 K\right)=p^{l} K, \frac{d \pi_{i}}{d K}=\frac{d p^{h}}{d K}\left(D^{h}-2 K\right)+p^{h}\left(D^{\prime} \frac{d p^{h}}{d K}-2\right)$. From $D^{\prime} \frac{d p^{h}}{d K}-2=$ $\frac{2 D^{\prime}}{2 D^{\prime}+p^{h} D^{\prime \prime}}-2=\frac{-2 D^{\prime}-2 p^{h} D^{\prime \prime}}{2 D^{\prime}+p^{h} D^{\prime \prime}}<0$ and $D^{h}-2 K<0$, we know that $\frac{d \pi_{i}}{d K}<0$. Using $\pi_{i}=p^{l} K$, we obtain $\frac{d \pi_{i}}{d K}=\frac{d p^{l}}{d K} K+p^{l}<0$, which implies $\frac{d p^{l}}{d K}<0$.

Lemma 1.2 states that the range of equilibrium prices $\left(p^{l}, p^{h}\right)$ shifts to the left as the capacity of each firm becomes larger. Intuitively this is because firms compete more aggressively when they have greater capacities.

\subsection{Merger Analysis}

Suppose firms $A$ and $B$ merge to form a new entity $M$ while firm $C$ remains unchanged. The merger leads to a duopoly market where one firm has a capacity twice as large as the other firm. To analyze the effects of this merger on profits and equilibrium 
prices, we will draw upon the findings from the existing studies of the asymmetric duopoly Bertrand-Edgeworth game by Kreps and Scheinkman (1983) and Osborne and Pitchik (1986).

The main findings from these two studies are that, when either firm's capacity is not too large $\left(K_{i}<D(0)\right)$ or both firms' capacities are not too small $\left(K_{i}>q_{i}\left(K_{j}\right)\right)$, firms will mix their price offers on the interval $\left(p^{L}, p^{H}\right)$. The larger firm, say firm $a$, determines the supremum $p^{H}$ by letting the smaller firm $b$ undercut it and acting as if it were a monopolist facing the residual demand $\left(D-K_{b}\right)$. Thus,

$$
p^{H}=\operatorname{argmax}_{p} p\left(D-K_{b}\right)
$$

and firm $a$ 's equilibrium profit is equal to $\pi_{a}=p^{H}\left(D\left(p^{H}\right)-K_{b}\right)$. On the other hand, firm $a$ determines the infimum $p^{L}$ by undercutting firm $b$ while earning equilibrium profit $\pi_{a}:$

$$
p^{L}= \begin{cases}\frac{\pi_{a}}{K_{a}} & \text { if } D\left(p^{L}\right)>K_{a} \\ \frac{\pi_{a}}{D\left(p^{L}\right)} & \text { if } D\left(p^{L}\right)<K_{a}\end{cases}
$$

Given the assumption that $p D$ is concave, the mixed strategy of firm $a, F_{a}$, is a continuous and increasing cumulative distribution function over the price range $\left(p^{L}, p^{H}\right]$. Firm $b$ takes this same range with open bound $\left(p^{L}, p^{H}\right)$ to mix its strategy, and earns equilibrium profit $\pi_{b}=p^{L} K_{b}$. Note that firm $b$ 's price never reaches $p^{H}$ because it always has an incentive to undercut its larger rival at this price. Also note that

$$
P\left(K_{a}+K_{b}\right)<p^{L}<p^{H}<P\left(K_{b}\right) .
$$

This is because it is not profitable for firm $a$ to set $p^{H}$ so high that the residual demand for itself is zero, or set $p^{L}$ so low to the level of competitive price. 
Finally since firm $a$ plays $p^{H}$ with a positive probability, its strategy $F_{a}$ everywhere

dominates $F_{b}$ over the range $\left(p^{L}, p^{H}\right)$. This suggests that the smaller firm competes more aggressively (by undercutting with greater probability) than the larger firm. We hereby let $p^{L}$ and $p^{H}$ denote the infimum and supremum of the post-merger price support respectively, if the equilibrium after merger turns out to be one in mixed strategy.

\subsubsection{Pure-Strategy Equilibrium before Merger}

Suppose that the equilibrium before the merger is one of pure strategies. Then before the merger each firm sets its price at $P(3 K)$ and earns a profit $K P(3 K)$. A key question in this case is, will firms play pure strategies after the merger?

To answer this question, we consider each firm's threshold for playing a mixed strategy after the merger. Since the merger does not change the capacity of firm C or the combined capacity of firm C's rival(s), the threshold for firm C to play a mixed strategy remains unchanged $\left(K>q_{i}(2 K)=\widetilde{K}_{C}=\widetilde{K}\right)$. For firm M, the total capacity of its rival is $K$ in comparison with $2 K$ that firms $\mathrm{A}$ and $\mathrm{B}$ each faces individually before the merger. Let $\widetilde{K}_{M}$ denote the threshold capacity of firm M above which it plays a mixed strategy.

Lemma 1.3 There exists a unique $\widetilde{K}_{M}$ such that

$$
\widetilde{K}_{M}=\operatorname{argmax}_{q} q D^{-1}\left(q+\widetilde{K}_{M} / 2\right) .
$$

Furthermore $\widetilde{K}_{M}<2 \widetilde{K}$.

- Proof: Using the same reasoning as in the proof of Lemma 1.1, we can prove the existence of a unique $\widetilde{K}_{M}$. Since the slope of the Cournot best-response function $\frac{d q\left(\sum K_{-i}\right)}{d \sum K_{-i}}=-\frac{P^{\prime}+q P^{\prime \prime}}{2 P^{\prime}+q P^{\prime \prime}}<0$ has an absolute value strictly less than 1 , a reduction in the combined capacity of the rival(s) $\left(\sum K_{-i}\right)$ by $K$ (from $2 K$ to $K$ ) would raise the value of 
$q\left(\sum K_{-i}\right)$ by less than $K$; in other words, $q(\widetilde{K})<2 \widetilde{K}$. Noting that $q(K / 2)<K$ if and only if $K>\widetilde{K}_{M}$, we have $\widetilde{K}_{M}<2 \widetilde{K}$.

Lemma 1.3 implies that if $K \leq \widetilde{K}_{M} / 2$, a pure-strategy equilibrium prevails after the merger and the equilibrium price remains at $P(3 K)$. In this case, the merger has no real effect on either the firms or consumers. If, on the other hand, $\widetilde{K}>K>\widetilde{K}_{M} / 2$, then mixed-strategy equilibrium prevails after the merger. Then (1.6) implies that $P(3 K)<$ $p^{L}<p^{H}<P(K)$. It immediately follows that firm $\mathrm{C}$ benefits from the merger because its expected profit increases from $P(3 K) K$ to $p^{L} K$. Firm $\mathrm{M}$, on the other hand, experiences the same kind of increase in profit (relative to the joint profits of its constituent firms $\mathrm{A}$ and $\mathrm{B})$, from $2 P(3 K) K$ to $2 p^{L} K$, as long as $p^{L} \leq P(2 K)$. But if $p^{L}>P(2 K)$, firm M's post-merger profit is $p^{L} D\left(p^{L}\right)$ where $D\left(p^{L}\right)<2 K$, in which case the impact of the merger on firm M's profit is not obvious. In spite of this, further investigation reveals that the merger is still profitable for the merging parties.

Proposition 1.1 If $\widetilde{K} \geq K>\widetilde{K}_{M} / 2$, the merger turns a pure-strategy equilibrium into a mixed-strategy equilibrium where the range of equilibrium prices are strictly higher than the pre-merger price. The merger is profitable for both the merging firms and the outside firm.

- Proof: Given the preceding discussion, we only need to show that the merger is profitable in the case $p^{L}>P(2 K)$. Note that $p^{L} D\left(p^{L}\right)>P(2 K) 2 K$ implies $p^{L} D\left(p^{L}\right)>$ $2 P(3 K) K$, because $P(2 K)>P(3 K)$. Recall that the expected profit of firm $\mathrm{M}$ is also equal to $p^{H} \cdot\left(D\left(p^{H}\right)-K\right)$. Optimization by firm $\mathrm{M}$ regarding $p^{H}$ yields the first-order condition $D\left(p^{H}\right)-K+p^{H} D^{\prime}\left(p^{H}\right)=0$, which implies $D\left(p^{H}\right)+p^{H} D^{\prime}\left(p^{H}\right)=K>0$. 
Noting that $D+p D^{\prime}$ is the first-order derivative of $p D$ and that $p D$ is concave, thus $\frac{d(p D)}{d p}>0$ for $p<p^{H}$. Then $p^{L}>P(2 K)$, implies $p^{L} D\left(p^{L}\right)>P(2 K) 2 K$.

One interesting observation from Proposition 1.1 is that in the case where the capacity constraints are binding (i.e., all firms produce at full capacity) in the pre-merger equilibrium, the merger can relax the capacity constraints. In the mixed-strategy equilibrium presented in Proposition 1.1, the quantities of both firms $\mathrm{M}$ and $\mathrm{C}$ are randomized over a range below their respective capacity levels. This, in turn, allows them to charge higher prices and earn larger expected profits after the merger.

\subsubsection{Mixed-Strategy Equilibrium before Merger}

A mixed strategy equilibrium prevails before the merger if $K>\widetilde{K}$. Since $\widetilde{K}>\widetilde{K}_{M} / 2$ , it follows that the equilibrium after the merger is one of mixed strategies as well. From Kreps and Scheinkman (1983) and Osborne and Pitchik (1986) we know that the postmerger $\operatorname{Supp}\left(F_{M}\right)=\left(p^{L}, p^{H}\right]$ and $\operatorname{Supp}\left(F_{C}\right)=\left(p^{L}, p^{H}\right)$, both fall within the range $(P(3 K), P(K))$.

\subsubsection{Change in the Supremum of the Price Support}

Lemma 1.4 In the case where $K>\widetilde{K}$, the supremum of the price support after the merger is greater than that before the merger, that is $p^{H}>p^{h}$.

- Proof: We know that $p^{H}=\operatorname{argmax}_{p} p \cdot(D(p)-K)$ and $p^{h}=\operatorname{argmax}_{p} p \cdot(D(p)-$ $2 K)$. So at point $p^{H},\left.\frac{d(p(D-K))}{d p}\right|_{p^{H}}=D\left(p^{H}\right)-K+p^{H} D^{\prime}\left(p^{H}\right)=0$ for firm M. Now evaluate the first-order derivative of firm A's or B's profit at the same point $p^{H}$ : $\left.\frac{d(p(D-2 K))}{d p}\right|_{p^{H}}=D\left(p^{H}\right)-2 K+p^{H} D^{\prime}\left(p^{H}\right)=D\left(p^{H}\right)-K+p^{H} D^{\prime}\left(p^{H}\right)-K=0-K=$ 
$-K<0$. Given that $p D$ is concave and that $p^{h}$ maximizes $p(D-2 K), p^{h}$ has to be less than $p^{H}$.

\subsubsection{Change in Firm M's Profit}

After the merger, the expected profit of firm $\mathrm{M}$ is equal to $\pi_{M}\left(p_{M}, F_{C}\right)=$ $F_{C}\left(p_{M}\right) p_{M}\left(D\left(p_{M}\right)-K\right)+\left(1-F_{C}\left(p_{M}\right)\right) p_{M} \min \left(2 K, D\left(p_{M}\right)\right)=p^{H}\left(D^{H}-K\right)$, where $D^{H} \equiv D\left(P^{H}\right)$. The expected profit of firm $\mathrm{C}$ is $\pi_{C}\left(p_{C}, F_{M}\right)$, which equals $F_{M} p_{c} \max \left\{D\left(p_{c}\right)-2 K, 0\right\}+\left(1-F_{M}\right) p_{c} K=p^{L} K$.

Recall that the expected profit of each firm before the merger is $\pi_{i}\left(p_{i}, F^{2}\right)=$ $\max _{p} p(D-2 K) \forall i \in\{A, B, C\}$. Since $\frac{d \pi}{d \sum K_{-i}}=\frac{d\left(p\left(D-\sum K_{-i}\right)\right)}{d \sum K_{-i}}=-p<0$, it is obvious that the profit of firm $\mathrm{M}$ is greater than that of an individual firm before the merger. But the more relevant comparison is between the profit of firm $\mathrm{M}$ and the sum of profits of two individual firms before the merger. In other words, is $p^{H} \cdot\left(D^{H}-K\right)>2 p^{h}$. $\left(D^{h}-2 K\right)$ ? The answer is yes.

Lemma 1.5 In the case of $K>\widetilde{K}$, the merger is profitable for firm M.

- Proof: Note that $p \cdot(D-K)>2 p \cdot(D-2 K)$ implies $D(p)<3 K$, which is true for each $p$ in the price support of firm M and C. Therefore, $p^{h} \cdot\left(D^{h}-K\right)>2 p^{h} \cdot\left(D^{h}-\right.$ $2 K)$. Since $p^{H}$ solves max $p \cdot(D-K)$, we have $p^{H} \cdot\left(D^{H}-K\right)>p^{h} \cdot\left(D^{h}-K\right)$. Hence $\pi_{M}>\pi_{A}+\pi_{B}$

\subsubsection{Change in the Infimum of the Price Support and in firm C's Profit}

Lemma 1.6 In the case where $K>\widetilde{K}, p^{L}>p^{l}$ and the merger benefits the outsider. 
-Proof: Note $p^{l}=\frac{\pi_{i}}{K}$ and $p^{L}=\frac{\pi_{M}}{\min (2 K, D)}$. In the case where $p^{L}<P(2 K), p^{L}=\frac{\pi_{M}}{2 K}>p^{l}$ because $\pi_{M}>2 \pi_{i}$ by Lemma 1.6. In the case where $p^{L}>P(2 K), p^{L}=\frac{\pi_{M}}{D\left(p^{L}\right)}>\frac{\pi_{M}}{2 K}$ (because $\left.D\left(p^{L}\right)<2 K\right)$, which is even greater than $p^{l}$. So in both cases $p^{L}>p^{l}$. Since $\pi_{C}\left(p_{C}, F_{M}\right)=p^{L} K$, the profit of the outsider (firm C) is higher after the merger.

Lemmas 1.4 and 1.6 suggest that the merger shifts the price support to the right. In Figure $1.1, F_{i}$ denotes the post-merger mixed strategy of firm $i(i=\mathrm{M}, \mathrm{C})$. Note that although $p^{h}$ has to be to the left of $P(2 K), p^{L}$ and $p^{H}$ could be on either side of $P(2 K)$. Figure 1.1 illustrates the positions of $F_{i}$ for three possible configurations of $P(2 K), p^{L}$ and $p^{H}$. In all three cases, $F_{i}$ lies to the right of the pre-merger mixed strategy $F$.

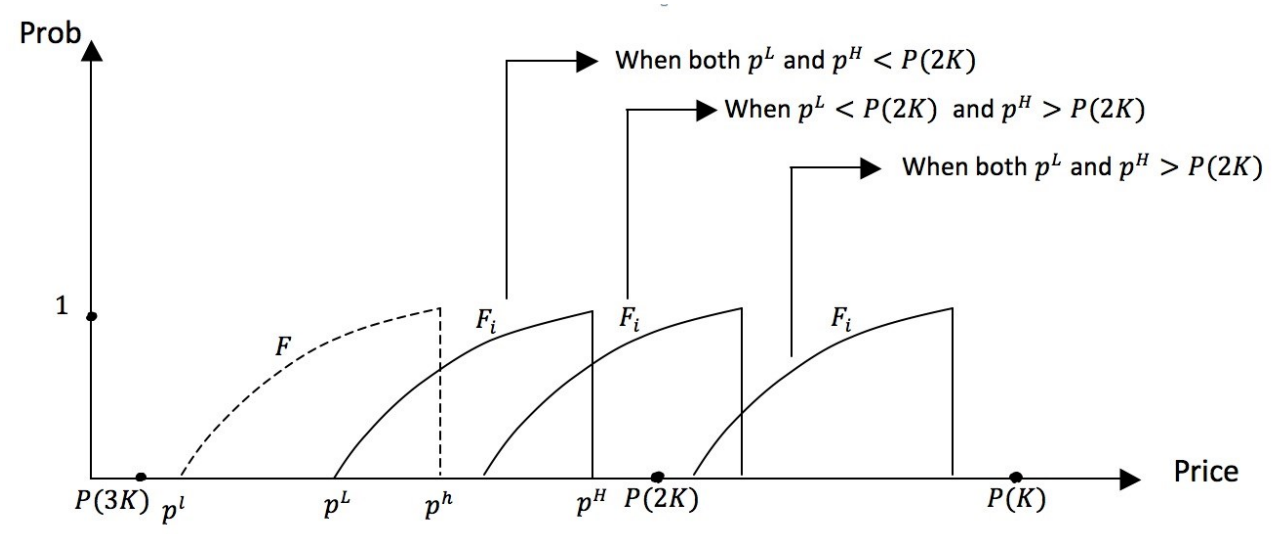

Figure 1.1 Equilibrium Strategies Before and After the Merger

\subsubsection{Change in the mixed strategies $F_{M}$ and $F_{C}$}

To solve the post-merger mixed strategies $F_{M}$ and $F_{C}$, we use the fact that a firm's expected profit is constant and maximized at each price level $p$ when the rival plays the equilibrium strategy. Depending on the magnitude of $P(2 K)$ relative to $p^{L}$ and $p^{H}$, there are three cases to consider. 
(1) In the case where $p^{L}<P(2 K)<p^{H}$, the expected profits of firm $\mathrm{M}$ and $\mathrm{C}$ can be expressed as follows.

$$
\begin{aligned}
\pi_{M}\left(F_{M}, F_{C}\right) & =\int_{p^{L}}^{p^{H}} \pi_{M}\left(p_{M}, F_{C}\right) d F_{M}=\int_{p^{L}}^{P(2 K)}\left[F_{C} p(D-K)+2\left(1-F_{C}\right) p K\right] d F_{M} \\
& +\int_{P(2 K)}^{p^{H}}\left[F_{C} p(D-K)+\left(1-F_{C}\right) p D\right] d F_{M}=p^{H}\left(D^{H}-K\right)=2 p^{L} K \\
\pi_{C}\left(F_{M}, F_{C}\right) & =\int_{p^{L}}^{p^{H}} \pi_{C}\left(p_{C}, F_{M}\right) d F_{C}=\int_{p^{L}}^{P(2 K)}\left[F_{M} p(D-2 K)+\left(1-F_{M}\right) p K\right] d F_{C} \\
& +\int_{P(2 K)}^{p^{H}}\left(1-F_{M}\right) p K d F_{C}=p^{L} K .
\end{aligned}
$$

Using the above equations, we find that the mixed strategies follow the probability distributions

$$
F_{M}=\frac{\left(p-p^{L}\right) K}{p(3 K-D)} \text { and } F_{C}=\frac{2\left(p-p^{L}\right) K}{p(3 K-D)}
$$

for prices in the range $p^{L}<p<P(2 K)$, and they follow

$$
F_{M}=1-\frac{p^{L}}{p} \text { and } F_{C}=\frac{p D-2 p^{L} K}{p K}
$$

for prices in the range $P(2 K)<p<p^{H}$.

Note that both $F_{M}$ and $F_{C}$ in (1.8) and (1.9) are continuous at price $P(2 K)$. This can be seen if we take the left limit of (1.8) and the right limit of (1.9), as follows. For $F_{M}$,

$$
\begin{gathered}
\lim _{p \rightarrow P(2 K)^{-}} F_{M}=\lim _{p \rightarrow P(2 K)^{-}} \frac{\left(p-p^{L}\right) K}{p(3 K-D)}=\frac{\left(P(2 K)-p^{L}\right) K}{P(2 K)(3 K-D(P(2 K)))}=\frac{P(2 K)-p^{L}}{P(2 K)} ; \\
\lim _{p \rightarrow P(2 K)^{+}} F_{M}=\lim _{p \rightarrow P(2 K)^{+}} 1-\frac{p^{L}}{p}=1-\frac{p^{L}}{P(2 K)}=\frac{P(2 K)-p^{L}}{P(2 K)} .
\end{gathered}
$$

For $F_{C}, \lim _{p \rightarrow P(2 K)^{-}} F_{C}=\lim _{p \rightarrow P(2 K)^{-}} \frac{2\left(p-p^{L}\right) K}{p(3 K-D)}=\frac{2\left(P(2 K)-p^{L}\right) K}{P(2 K)(3 K-D(P(2 K)))}=\frac{2 P(2 K)-2 p^{L}}{P(2 K)}$

$$
\lim _{p \rightarrow P(2 K)^{+}} F_{C}=\lim _{p \rightarrow P(2 K)^{+}} \frac{p D-2 p^{L} K}{p K}=\frac{P(2 K) D(P(2 K))-2 p^{L} K}{P(2 K) K}=\frac{2 P(2 K)-2 p^{L}}{P(2 K)} .
$$


(2) In the case where $p^{H}<P(2 K)$ (and hence $p^{L}<P(2 K)$ ), each firm's expected profit is equal to:

$$
\begin{aligned}
& \pi_{M}\left(F_{M}, F_{C}\right)=\int_{p^{L}}^{p^{H}} \pi_{M}\left(p_{M}, F_{C}\right) d F_{M}=\int_{p^{L}}^{p^{H}}\left[F_{C} p(D-K)+2\left(1-F_{C}\right) p K\right] d F_{M}=2 p^{L} K \\
& \pi_{C}\left(F_{M}, F_{C}\right)=\int_{p^{L}}^{p^{H}} \pi_{C}\left(p_{C}, F_{M}\right) d F_{C}=\int_{p^{L}}^{p^{H}}\left[F_{M} p(D-2 K)+\left(1-F_{M}\right) p K\right] d F_{C}=p^{L} K .
\end{aligned}
$$

We use these equations to solve for the equilibrium mixed strategies:

$$
F_{M}=\frac{\left(p-p^{L}\right) K}{p(3 K-D)} \text { and } F_{C}=\frac{2\left(p-p^{L}\right) K}{p(3 K-D)}
$$

(3) In the case where $p^{L}>P(2 K)$, the expected profit of each firm is given by

$$
\begin{aligned}
\pi_{M}\left(F_{M}, F_{C}\right) & =\int_{p^{L}}^{p^{H}} \pi_{M}\left(p_{M}, F_{C}\right) d F_{M}=\int_{p^{L}}^{p^{H}}\left[F_{C} p(D-K)+\left(1-F_{C}\right) p D\right] d F_{M} \\
& =p^{H}\left(D^{H}-K\right)=p^{L} D^{L} ; \\
\pi_{C}\left(F_{M}, F_{C}\right) & =\int_{p^{L}}^{p^{H}} \pi_{C}\left(p_{C}, F_{M}\right) d F_{C}=\int_{p^{L}}^{p^{H}}\left(1-F_{M}\right) p K d F_{C}=p^{L} K .
\end{aligned}
$$

It can be shown that the mixed strategies follow the probability distributions:

$$
F_{M}=1-\frac{p^{L}}{p}, F_{C}=\frac{p D-p^{L} D^{L}}{p K}
$$

Recall that a firm's mixed strategy before the merger is given by $F=\sqrt{\frac{\left(p-p^{l}\right) K}{p(3 K-D)}}$. By comparing this with the mixed strategies after the merger, we obtain the following proposition.

Proposition 1.2 In the case where $K>\widetilde{K}$, post-merger price distributions $F_{M}$ and $F_{C}$ strictly dominate pre-merger price distribution $F$ stochastically at first order. In other words, pre-merger firms have strictly greater probability to sell below any particular price than either of the post-merger firms does (except at $p^{H}$ ). Accordingly, the merger is profitable for the merged entity and the outside firm. 
The proof of Proposition 1.2 is long and tedious. Hence it is relegated to the appendix. Here we summarize our finding about this case by noting that the merger reduces the competition intensity between firms. While a mixed-strategy equilibrium prevails before and after the merger, the range of prices shifts upward and the probability of price falling below any particular level is lower after the merger.

\subsubsection{Comments}

We end this section by comparing the results we have obtained so far with those in Froeb, et al. (2003). For this purpose, it is useful to remind us what it means for a firm to be "capacity constrained". In the Bertrand-Edgeworth model, a firm is capacity constrained if its production capacity is not large enough to supply the whole market at price equal to marginal cost, or using the notations in our model, if $K<D(0)$. It is the presence of this capacity constraint that creates the possibility of other equilibrium outcomes than the standard Bertrand equilibrium. Note, however, a firm's capacity constraint is not always binding in Bertrand-Edgeworth equilibrium. In a mixed-strategy equilibrium, a firm produces at full capacity only with some probability.

In our model, the assumption $2 K<D(0)$ ensures that every firm is capacity constrained before and after the merger. Our analysis shows that the merger leads to higher prices as long as a mixed strategy prevails after the merger. The merger has no effect on equilibrium prices if and only if capacity is so small that a pure-strategy equilibrium prevails both before and after the merger.

Froeb, et al. (2003), on the other hand, examine situations where some firms are capacity constrained while others are not. In particular, they obtain the result that a merger has no price effect in situations where the merging firms are capacity constrained 
but the outside firms are not. Froeb, et al. (2003) attribute the absence of merger effect to the capacity constraints of the merging firms. But the real reason, in our view, is that each outside firm has a capacity large enough to supply the entire demand $D(0)$. This destroys the merged entity's incentives to raise price above its rival's because doing so would leave itself zero residual demand.

\subsection{A Model with Linear Demand Function}

In this section, we illustrate our results using a linear demand function $p=a-b Q$. It is easy to verify that $p D(p)=p(a-p) / b$ is concave in $p$. Our assumption on the capacity constraint requires that $2 K<D(0)=\frac{a}{b}$.

\subsubsection{Pre-Merger Equilibrium}

The threshold capacity for a mixed-strategy equilibrium is determined by $\operatorname{argmax}_{q} q(a-b(q+2 K))$, from which we obtain $\widetilde{K}=a /(4 b)$. Then a pure-strategy equilibrium prevails if $K \in\left(0, \frac{a}{4 b}\right]$, in which case each firm sets $p=P(3 K)=a-3 b K$ and earns a profit equaling $a K-3 b K^{2}$. On the other hand, if $K \in\left(\frac{a}{4 b}, \frac{a}{2 b}\right)$, the game has a mixed-strategy equilibrium over the price range $\left(p^{l}, p^{h}\right)$, where

$$
p^{h}=\operatorname{argmax}_{p} p(D-2 K)=\operatorname{argmax}_{p} p\left(\frac{a-p}{b}-2 K\right)=\frac{a}{2}-b K
$$

and

$$
p^{l}=\frac{\pi_{i}}{K}=\frac{p^{h}\left(D^{h}-2 K\right)}{K}=\frac{1}{K}\left(\frac{a}{2}-b K\right)\left(\frac{a-\frac{a}{2}+b K}{b}-2 K\right)=\frac{1}{b K}\left(\frac{a}{2}-b K\right)^{2}
$$

It is easy to show that $p^{h}<P(K)=a-b K$ and $p^{l}>P(3 K)=a-3 b K$. The profit of each firm in a mixed-strategy equilibrium is equal to $p^{l} K=\frac{1}{b}\left(\frac{a}{2}-b K\right)^{2}$. 
The equilibrium mixed strategy $F$ is determined by the fact that expected profit of any firm $\pi_{i}\left(p_{i}, F_{-i}\right)$ is equal at every $p$ in the support, that is,

$$
F^{2} p \cdot(D-2 K)+\left(1-F^{2}\right) p K=\frac{1}{b}\left(\frac{a}{2}-b K\right)^{2} .
$$

Solving the above equation for $F$, we obtain: $F=\sqrt{\frac{b p K-\left(\frac{a}{2}-b K\right)^{2}}{p(3 b K-a+p)}}$.

\subsubsection{Merger Analysis}

Suppose firms A and B merge, the new entity firm $M$ has capacity $2 K$ and firm $\mathrm{C}$ still has capacity $K$. Firm C's mixed strategy threshold remains the same as before the merger: $\widetilde{K}_{C}=a /(4 b)$. But firm M's mixed strategy threshold is now determined by $\widetilde{K}_{M}=\operatorname{argmax}_{q} q\left(a-b\left(q+\widetilde{K}_{M}\right)\right)$. Solving this equation yields: $\widetilde{K}_{M}=2 a / 5 b$.

Note that $\frac{\widetilde{K}_{M}}{2}=\frac{a}{5 b}<\widetilde{K}_{C}$. Hence, if $K \leq \frac{a}{5 b}$, the game has a pure-strategy equilibrium at the price $p=P(3 K)=a-3 b K$. In this case, the merger has no real effect.

If the capacity is larger than $\frac{a}{5 b}$, the post-merger equilibrium is one of mixedstrategies, and the merger has effects on equilibrium price and quantities. Depending on the magnitude of $K$, the equilibrium strategies are different. As shown in Appendix B, we can divide the range of $K$ into three segments.

$$
\begin{aligned}
& \text { First, if } K \in\left(\frac{a}{5 b}, \frac{a}{3 b}\right) \text {, the post-merger equilibrium strategies are given by } \\
& F_{M}=\frac{8 b p K-(a-b K)^{2}}{8 p(3 b K-a+p)}, \text { and } F_{C}=\frac{8 b p K-(a-b K)^{2}}{4 p(3 b K-a+p)} .
\end{aligned}
$$

The supremum and infimum of the equilibrium prices are, respectively, $p^{H}=\frac{a-b K}{2}$, and $p^{L}=\frac{1}{8 b K}(a-b K)^{2}$. Note that for $K$ in this range, the pre-merger equilibrium may be one of pure strategies. Specifically, if $K \in\left(\frac{a}{5 b}, \frac{a}{4 b}\right)$, a pure-strategy equilibrium prevails 
before the merger, and the merger turns the equilibrium into one of mixed strategies. If $K \in\left(\frac{a}{4 b}, \frac{a}{3 b}\right)$, on the other hand, a mixed-strategy equilibrium prevails both before and after the merger.

Second, if $K \in\left(\frac{a}{3 b}, 0.46 \frac{a}{b}\right)$, the supremum and infimum of the equilibrium price are the same as above, i.e., $p^{H}=\frac{a-b K}{2}$ and $p^{L}=\frac{1}{8 b K}(a-b K)^{2}$. But the equilibrium strategies have to be stated in two separate pieces. Specifically, the equilibrium strategies follow the distributions

$$
F_{M}=\frac{8 b p K-(a-b K)^{2}}{8 p(3 b K-a+p)}, \quad \text { and } \quad F_{C}=\frac{8 b p K-(a-b K)^{2}}{4 p(3 b K-a+p)}
$$

for prices in the range $\left(p^{L}, a-2 b K\right)$, and they follow

$$
F_{M}=1-\frac{1}{8 b p K}(a-b K)^{2}, \text { and } \quad F_{C}=\frac{4 p(a-p)-(a-b K)^{2}}{4 b p K}
$$

for prices in the range $\left(a-2 b K, p^{H}\right)$. In this case, it can be shown that $\left.F_{M}\right|_{p^{H}}<1$, which means that firm M plays $p^{H}$ with positive probability.

Third and finally, if $K \in\left(0.46 \frac{a}{b}, \frac{a}{2 b}\right)$, the supremum and infimum of the equilibrium price are given by $p^{H}=\frac{a-b K}{2}, p^{L}=\frac{a}{2}-\frac{1}{2} \sqrt{(2 a-b K) b K}$, and the equilibrium strategies follow the distributions

$$
F_{M}=1-\frac{a}{2 p}+\frac{1}{2 p} \sqrt{(2 a-b K) b K} \text {, and } \quad F_{C}=\frac{1}{p b K}\left(a p-p^{2}-a p^{L}+p^{L^{2}}\right) .
$$

It can be shown that in all three cases, the merger raises equilibrium prices and improves the profits of both the insiders and the outsider. 


\subsection{An Extension}

In this section, we extend our analysis to a more general case where there are $n(\geq 3)$ identical firms before the merger. A Bertrand-Edgeworth model with a large market is studied in Allen and Hellwig (1986) who confirm the existence of both pure and mixed strategies and characterize their properties. Using a different rationing rule, they prove that the upper bound of price support could be reached with positive probability by at most one of the firms. ${ }^{8}$ Further studies by Vives (1986, Proposition 1) and Francesco and Salvadori (2009) characterize the equilibrium in $n$-firm market with symmetric and asymmetric capacity constraints, respectively. Note that both of these studies both use the same rationing rule as ours, thus expedite our effort to extend our 3 -firm model to $n$-firm model. None of the three papers, however, study mergers, which would involve the comparison of equilibriums before and after the combination of two firms.

We assume $(n-1) K<D(0)$ to prelude the standard Bertrand equilibrium with price equaling marginal cost. All other aspects of the model remain the same as in section 1.2.

Most of our results from the three-firm model can be extended to the $n$-firm case. Since most proofs are similar to those in the three-firm case, here we will not go through every detail of the $n$-firm model, but focus, instead, on the presentation of main results.

\subsubsection{Pre-Merger Equilibrium}

As before, let $q\left(\sum K_{-i}\right)$ denote a firm's Cournot best-response function when the combined capacity of its rivals is $\sum K_{-i}$. Using the same procedure as in the proof of Lemma 1.1 , we can establish that there exists a unique $\widehat{K}$ such that $\widehat{K}=q((n-1) \widehat{K})$,

\footnotetext{
${ }^{8}$ See Proposition 3.6, p.183.
} 
and that $K \leq q((n-1) K)$ if and only if $K \leq \widehat{K}$. Accordingly, a pure-strategy equilibrium prevails before the merger if $K \leq \widehat{K}$. In this case, the equilibrium price is $P(n K)$, and the profit is $P(n K) K$ for each firm.

If $K>\widehat{K}$, on the other hand, mixed-strategy equilibrium prevails before the merger. The supremum and infimum of the equilibrium price support are determined by $p^{h}=\operatorname{argmax}_{p} p(D-(n-1) K)$ and $p^{l}=\frac{p^{h}\left(D^{h}-(n-1) K\right)}{K}$, where $P(n K)<p^{l}<p^{h}<$ $P((n-1) K)$. From $\pi_{i}\left(p_{i}, F^{n-1}\right)=F^{n-1} p(D-(n-1) K)+\left(1-F^{n-1}\right) p K=p^{l} K$, we obtain the equilibrium strategy of each firm $F=\left(\frac{\left(p-p^{l}\right) K}{p(n K-D)}\right)^{\frac{1}{n-1}}$. It can be shown that as $n$ increases, the price support shifts to the left, and the profit of each firm falls. Intuitively, an increase in the number of firms (and a larger total production capacity) intensifies competition, thus reducing the prices and profits.

\subsubsection{Merger Analysis}

As before, we call the entity formed by the merger of two firms firm M. Note that the number of outside firms is now $(n-2)$. Since we have already examined the case of $n=3$, here we suppose $n \geq 4$. Accordingly, there are at least two outside firms after the merger. ${ }^{9}$

\subsubsection{Pure-Strategy Equilibrium before Merger}

Following the proof of Lemma 1.4, we can establish that there exists a unique $\widehat{K}_{M}$ such that $\widehat{K}_{M}=q\left((n-2) \widehat{K}_{M} / 2\right)$, and $\widehat{K}_{M} \leq q\left((n-2) \widehat{K}_{M} / 2\right)$ if and only if $K \leq \widehat{K}_{M} / 2$. Furthermore $\widehat{K}>\widehat{K}_{M} / 2$.

\footnotetext{
${ }^{9}$ This implies in equilibrium the demand never falls below $2 K$. This simplifies the analysis on what happens when firm $\mathrm{M}$ undercuts its rivals.
} 
Therefore, the post-merger equilibrium will remain one of pure strategies if $K \leq$ $\widehat{K}_{M} / 2$, in which case the merger has no real effect. On the other hand, if $K$ is in the range $\left(\widehat{K}_{M} / 2, \widehat{K}\right]$, the merger leads to a mixed-strategy equilibrium, with $P(n K)<p^{L}<p^{H}<$ $P((n-2) K)$. Following the proof of Proposition 1.1, we can show that the merger improves the profits of both insiders and outsiders.

\subsubsection{Mixed-Strategy Equilibrium before Merger}

Here we consider the case $K>\widehat{K}$. From the discussion above, we know that the postmerger equilibrium is one of mixed strategies as well. Because $\frac{d p^{H}}{d \sum K_{-i}}=\frac{1}{2 D^{\prime}+p D^{\prime \prime}}<0, p^{H}$ increases when the rivals' total capacity decreases from $(n-1) K$ to $(n-2) K$ after the merger, so $p^{H}>p^{h}$. Furthermore, $p^{L}>P(n K)$ implies that $p \cdot(D-(n-2) K)>2 p$. $(D-(n-1) K)$. Then we have $p^{H} \cdot\left(D^{H}-(n-2) K\right)>p^{h} \cdot\left(D^{h}-(n-2) K\right)>2 p^{h}$. $\left(D^{h}-(n-1) K\right)$; in other words, firm M's profit is greater than the combined premerger profits of the two merging firms. This, in turn, implies that $p^{L} 2 K>2 p^{l} K$, or equivalently $p^{L}>p^{l}$.

The equilibrium strategies depend on the magnitude of $P((n-1) K)$ relative to $p^{H}$ and $p^{L}$. In the appendix, we examine the equilibrium profits of firm $\mathrm{M}$ and each outside firm, and use them to find the equilibrium strategy for the case where $p^{H}<P((n-$ 1)K):

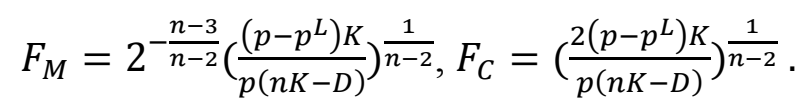

For the case $p^{L}<P((n-1) K)<p^{H}$, however, we are able to obtain a solution for only a part of the mixed strategies. Specifically, we find that $F_{M}$ and $F_{C}$ are the same as (1.12) for prices in the range $p^{L}<p<P((n-1) K)$. We are not able to obtain an explicit 
solution for prices in the range $P((n-1) K)<p<p^{H}$. Neither can we find the solution for the case $p^{L}>P((n-1) K)$. Despite that, we are able to prove that the post-merger price distributions $F_{M}$ and $F_{C}$ stochastically dominate the pre-merger distribution $F$ (see Appendix C).

Therefore, we can summarize our findings for the $n$-firm model as follows:

Proposition 1.3 In the model with $n$ firms,

(1) If $\widehat{K} \geq K>\widehat{K}_{M} / 2$, the merger turns a pure-strategy equilibrium into a mixedstrategy equilibrium where the range of equilibrium prices are strictly higher than the pre-merger price. The merger is profitable for both the merging firms and the outside firm.

(2) If $K>\widehat{K}$, post-merger price distributions $F_{M}$ and $F_{C}$ strictly dominate pre-merger price distribution F stochastically at first order. In other words, pre-merger firms have strictly greater probability to sell below any particular price than either of the postmerger firms does (except at $p^{H}$ ). Accordingly, the merger is profitable for the merged entity and the outside firm.

In other words, all of the results from the three-firm model in Propositions 1.1 and 1.2 continue to hold in the $n$-firm model.

\subsection{Concluding Remarks}

We have examined the effects of a merger between two firms in a BertrandEdgeworth model. We have shown that if the production capacity of each firm is sufficiently small that a pure-strategy equilibrium prevails both before and after the merger, the merger has no effect on either prices or profits. However, a larger capacity 
leads to mixed-strategy equilibrium after the merger, in which case the merger raises the prices and improves the profitability of all firms. In the case where mixed-strategy equilibrium prevails both before and after the merger, the post-merger price distribution of each firm stochastically dominates the pre-merger distribution.

A couple of assumptions in our model are particularly worth noting. First, we have assumed that all firms have the same capacity before the merger. This has simplified our analysis by substantially reducing the number of possible cases we have to consider. At the same time, it probably also eliminated some forces that may be at play when firms are asymmetric. Second, we have adopted a standard Bertrand-Edgeworth where all firms produce a homogeneous product. Allowing product differentiation will likely influence the effects of a merger. Deneckere and Davidson (1985) have shown that a merger leads to higher prices when firms produce differentiated products. It will be interesting to investigate whether the presence of capacity constraints strengthens or mitigates this price effect. 


\section{Chapter 2: Horizontal Merger in a Cournot Model with Differentiated Products}

\subsection{Introduction}

Consideration of product differentiation figures prominently in merger enforcement by competition authorities around the world. For example, the US Horizontal Merger Guidelines (thereafter Guidelines) acknowledge, "Some substitutes may be closer, and others more distant, either geographically or in terms of product attributes and perceptions. Additionally, customers may assess the proximity of different products differently" (section 4). While product differentiation used to be only a mitigating factor in the 1982/84 Guidelines, it has received more and more attention since the 1992 Guidelines, especially in the new 2010 Guidelines where the concept of diversion ratio was officially introduced into merger analysis. In the theoretical literature, however, most merger analyses involving product differentiation are conducted under the assumption of price competition, while studies of mergers under quantity competition are relatively rare and incomplete.

In this chapter we examine how the effects of merger (such as prices and profits) are influenced by the degree of product differentiation under the assumption that firms compete in quantity. By allowing the degree of substitutability to differ among different products we show that the conclusions from some of the existing studies could be misleading due to their simplicity of assumptions while our findings are more complete and in better accordance with received economic rationale and empirical results. Furthermore we study how these degrees of differentiation between products affect merger effects. 
An important strand of theoretical literature on merger analysis studies the profitability of mergers. As has been observed by Salant, et al. (1983), a merger among firms that compete in quantity in a homogeneous good market is not profitable unless it involves $80 \%$ or more of the firms in the market. This result, termed as the merger paradox, has also been found in Szidarovszky and Yakowitz (1982), Matsushima (2001) and Hirata (2009). ${ }^{11}$ Ever since Salant, et al. (1983), a number of authors have endeavored to resolve this paradox. One approach was to modify certain aspects of the quantity competition model. In particular, Daughety (1990) shows that if firms choose quantity sequentially, merger could be profitable (and welfare enhancing) even without cost synergies. Perry and Porter (1985), followed by McAfee and Williams (1992), Heywood and McGinty (2007), resolve the merger paradox by assuming that firms face rising marginal cost. Davidson and Mukherjee (2007) show that merger is always profitable with free entry as long as there is (any degree of) cost synergy. ${ }^{12}$

Another approach to the examination of merger profitability is to assume that firms compete in prices in a market of differentiated products. Specifically, Deneckere and Davidson (1985) use a non-spatial differentiated product Bertrand model to show that merger will lead to higher prices and profits. Other authors used spatial models of product differentiation to allow for localized competition. Reitzes and Levy (1990) and Levy and Reitzes (1993), for example, demonstrate that the effects of merger are stronger

\footnotetext{
${ }^{11}$ However, empirical studies typically find increased post-merger prices or market power. For example, Weinberg (2008) surveys empirical literature on price effects of merger and finds that in most cases studied, mergers led to higher prices. Baker and Bresnahan (1985) predict that a merger between brewing firms Anheuser-Busch and either Pabst or Coors would enhance the former's market power.

${ }^{12}$ Also related are Cheung (1992) and Faulí-Oller (1997) who show that merger profitability decreases as demand becomes more concave.
} 
under localized competition than under non-spatial competition. ${ }^{13}$ Moreover, Reitzes and Levy (1990) and Levy and Reitzes (1992) show that merger is profitable for firms on a circle with fixed locations. Braid $(1986,1999)$ draws the same conclusion using a spatial model with a straight line and a two-dimensional spatial model, respectively. Reitzes and Levy (1995) show merger profitability in a more general spatial setting when firms are able to engage in price discrimination.

A third approach to the study of merger profitability is to assume that firms compete in quantity in a market of differentiated products. This approach has been taken by a small number of authors. Specifically, Erkal and Piccinin (2010) find that entryinducing merger may be profitable even when cost synergies are not strong enough. ${ }^{14}$ The two studies that are closest to the present chapter are Ebina and Shimizu (2009) and Hsu and Wang (2010). Both studies are based on a quantity-competition model with only four firms. By allowing the degree of product differentiation to differ among different pairs of firms, Ebina and Shimizu (2009) find that merger incentives are stronger for two firms producing closer substitutes than for those producing more remote substitutes. Hsu and Wang (2010), on the other hand, assume that all four firms have the same degree of substitutability for each other, and obtained a rather unintuitive result that merger is profitable when merging products are more distant substitutes.

The literature mentioned above assumes that firms take no actions other than pricing or output decisions. However there are other dimensions of strategies, and

\footnotetext{
${ }^{13}$ They also explain merger effects in terms of how localized competition fosters collusion. Also see Davidson and Deneckere (1984) and Baker (1997).

${ }^{14}$ In addition, M'Chirgui and Hichri (2006) show that when downstream duopoly firms merge into a monopoly the merger is profitable if product substitutability is medium. However, a merger of duopoly to a monopoly is profitable even in the original model by Salant, et al. (1983).
} 
product repositioning is one of these. It has long been held that non-merging firms tend to respond to merger by differentiating or repositioning products closer to merging firms' product space, thus constraining the price-increase and mitigating some of the anticompetitive effects, as the 1997 Guidelines acknowledges (section 2.212). Indeed, some recent studies have studied firms' product-repositioning decisions. The literature includes Norman and Pepall (2000) with Cournot competition and Gandhi, et al. (2008) with Bertrand competition. Both yield similar observations that merging firms tend to differentiate and move away from the center point of a linear market while non-merging firms tend to move towards or cluster at the center point. These ideas have been encompassed in the 2010 Guidelines (section 6.4). In both analyses, mergers are profitable due to reduced competition.

In this chapter, we investigate the effects of merger using a more general model than Ebina and Shimizu (2009) and Hsu and Wang (2010). In our model, $N$ firms produce differentiated products, and the two merging firms (the insiders) have a different degree of substitutability between themselves than between each insider and other firms (the outsiders). Moreover, both of these degrees of substitution can be different from that between any pair of outsiders to the merger.

Using this model, we examine both the conditions for profitable mergers and the impact of the degree of substitutability among products on merger profitability. We find that product differentiation can resolve the merger paradox associated with quantity competition by broadening the range of parameters over which a merger is profitable. To be more specific, a merger is profitable if the number of competitors is small, and if the substitutability is high between the two insiders but low between each insider and the 
outsiders. The post-merger prices of the insiders are higher when the substitutability is higher between the two insiders or lower between each insider and the outsiders.

The remainder of this chapter is arranged as follows. Section 2.2 sets up the basic model and examines pre-merger equilibrium; and section 2.3 analyzes merger equilibrium and derives conditions for profitable merger as well as merger effects. Section 2.4 closes the chapter.

\subsection{The Model}

Consider a market where $N$ firms produce differentiated products and compete in quantities. ${ }^{15}$ We assume that two potential merging firms, namely 1 and 2, produce closer or more distant substitutes than the rest of the firms. The remaining firms, the outsiders to the potential merger, are denoted by $i, j \in\{3, \ldots, N\}$. To focus on the impact of product substitutability on a two-firm merger we let other firms be symmetric. There are no fixed cost and all firms have the same marginal cost $c$ both before and after merger.

To be more specific, we assume a linear demand system:

$$
\begin{aligned}
& p_{1}=\alpha-q_{1}-\gamma q_{2}-\delta \sum_{j=3}^{N} q_{j} \\
& p_{2}=\alpha-\gamma q_{1}-q_{2}-\delta \sum_{j=3}^{N} q_{j} \\
& p_{i}=\alpha-q_{i}-\varphi \sum_{j \neq i} q_{j}(i=3,4, \ldots, N)
\end{aligned}
$$

where $0<\max \{\gamma, \delta, \varphi\}<1$.

\footnotetext{
${ }^{15}$ See footnote 3 in McAffee, et al. (1992) for a discussion about the advantages of Cournot model against Bertrand or other cartel models.
} 
To simplify presentation, we define $A \equiv(N-3) \varphi+2$ and $B \equiv(N-2) \delta$. We assume that $A>B$ and $\alpha>c$ to ensure that prices and quantities are positive in the premerger equilibrium. ${ }^{16} \mathrm{~A}$ sufficient condition for $A>B$ to hold is $\varphi>\delta$.

In this setting firms 1 and 2 regard each other as closer (respectively, more distant) competitors than the rest of the firms when $\gamma>\delta$ (respectively, $\gamma<\delta$ ). For each of the other firms, however, its demand is equally affected by the quantities of all competitors. ${ }^{17}$ We make these assumptions to focus on the role played by the substitutability between the two merging firms relative to the substitutability between any other pairs of products.

\subsubsection{Pre-merger Equilibrium}

Firms maximize profits by choosing output levels. Firm 1's optimization problem is

$$
\max _{q_{1}}\left(p_{1}-c\right) q_{1}
$$

and the first order condition gives

$$
\alpha-2 q_{1}-\gamma q_{2}-\delta \sum_{j=3}^{N} q_{j}=c .
$$

Firm 2's optimization problem is

$$
\max _{q_{2}}\left(p_{2}-c\right) q_{2}
$$

with the first order condition

$$
\alpha-\gamma q_{1}-2 q_{2}-\delta \sum_{j=3}^{N} q_{j}=c .
$$

Firm $i$ 's optimization problem is

$$
\max _{q_{i}}\left(p_{i}-c\right) q_{i}
$$

and the first order condition gives

\footnotetext{
${ }^{16}$ The rationale for the assumption $A>B$ is explained below in section 2.2.1. This assumption is not necessary in the more symmetric case of $\varphi=\delta$, where $A-B$ is always positive.

${ }^{17}$ Note that in our model we allow asymmetry in the demand functions of insiders and outsiders: While the marginal impact of firm 1's quantity on firm $i$ 's $(i \neq 1,2)$ price is $\delta$, that of firm $i$ 's quantity on firm 1 's price is $\varphi$. This setup includes the symmetric demand functions where $\delta=\varphi$ as a special case.
} 


$$
\alpha-2 q_{i}-\varphi \sum_{j \neq i} q_{j}=c .
$$

Solving the equation system formed by (2.5), (2.7) and (2.9) we obtain the equilibrium quantities, prices, and profits:

$$
\begin{aligned}
& q_{1}^{*}=q_{2}^{*}=\frac{[2+(N-3) \varphi-(N-2) \delta](\alpha-c)}{(2+\gamma)[(N-3) \varphi+2]-2(N-2) \varphi \delta}=\frac{(A-B)(\alpha-c)}{(2+\gamma) A-2 \varphi B} \\
& q_{i}^{*}=\frac{(2+\gamma-2 \varphi)(\alpha-c)}{(2+\gamma)[(N-3) \varphi+2]-2(N-2) \varphi \delta}=\frac{(2+\gamma-2 \varphi)(\alpha-c)}{(2+\gamma) A-2 \varphi B} \\
& p_{1}^{*}=p_{2}^{*}=\frac{(\alpha+c+c \gamma) A+(c-\alpha-2 c \varphi) B}{(2+\gamma) A-2 \varphi B}=q_{1}^{*}+c \\
& p_{i}^{*}=\frac{c(2+\gamma) A-2 \varphi c B+(\alpha-c)(2+\gamma-2 \varphi)}{(2+\gamma) A-2 \varphi B}=q_{i}^{*}+c \\
& \pi_{1}^{*}=\pi_{2}^{*}=\frac{(\alpha-c)^{2}(A-B)^{2}}{[(2+\gamma) A-2 \varphi B]^{2}}=\left(q_{1}^{*}\right)^{2} \\
& \pi_{i}^{*}=\frac{(\alpha-c)^{2}(2+\gamma-2 \varphi)^{2}}{[(2+\gamma) A-2 \varphi B]^{2}}=\left(q_{i}^{*}\right)^{2}
\end{aligned}
$$

Before we proceed to interpret our results we need to make sure that all our equilibrium prices and quantities are positive, which is indeed the case with assumptions that that $\alpha>c$ and $A>B .{ }^{19}$ Note the prices and quantities of firm $i(i=3, \ldots, N)$ are always positive even without the assumption $A>B$. From equation (2.10) we can see the values of $\varphi$ have to be great enough relative to $\delta^{20}$ to ensure that firm 1 and 2's quantities are positive. Intuitively $\varphi$ measures firm 1's ability to affect firm $i$ 's profit and $\delta$ measures the influence in the other direction. A greater $\delta$ means firm 1's price is forced down more when firm $i$ sets higher outputs, making firm 1 more open to competition from firm $i$ while a smaller $\varphi$ means it exerts less influence on firm $i$, and is therefore put in greater disadvantage competing against firm $i$. This disadvantage is aggravated with

\footnotetext{
${ }^{19}$ Note the common denominator $(2+\gamma)[(N-3) \varphi+2]-2(N-2) \varphi \delta$ is easily arranged as $2(N-2) \varphi(1-\delta)+(N-2) \varphi \gamma+(2+\gamma)(2-\varphi)$, which is positive independent of the assumption $A>B$.

${ }^{20}$ But note that $\varphi$ could be either greater or less than $\delta$.
} 
more competitors: when $N \leq 4, A-B$ will be positive no matter how $\varphi$ and $\delta$ compare, but if $N>5, A-B$ could be negative. Finally, note that $\gamma$ does not appear in $A$ and $B$ and hence does not affect the sign of (2.10). This is because $\gamma$ affects the demand facing firms 1 and 2 symmetrically.

\subsubsection{Comparative Statics in Pre-merger Equilibrium}

We conduct comparative statics to find out how the equilibrium is affected by the degree of demand substitutability in this industry. With respect to firms 1 and 2 , it is obvious that $\frac{\partial q_{1}^{*}}{\partial \gamma}, \frac{\partial p_{1}^{*}}{\partial \gamma}$ and $\frac{\partial \pi_{1}^{*}}{\partial \gamma}<0$ (since $\gamma$ appears only in denominators), meaning the higher the degree of substitutability between firms 1 and 2, the lower the equilibrium outputs, prices and profits. Intuitively with greater $\gamma$ firms 1 and 2 compete more intensely against each other and suffer the consequences of lower prices and quantities. Next, it can be shown that $\frac{\partial q_{1}^{*}}{\partial \delta}, \frac{\partial p_{1}^{*}}{\partial \delta}$ and $\frac{\partial \pi_{1}^{*}}{\partial \delta}<0,{ }^{21}$ which implies that equilibrium outputs, prices and profits fall with greater $\delta$. A greater $\delta$ means that firms 1 and 2 face more intense competition from firm $i(=2,3, \ldots, N)$, hence the lower profits. Thirdly, we find that $\frac{\partial q_{1}^{*}}{\partial \varphi}, \frac{\partial p_{1}^{*}}{\partial \varphi}$ and $\frac{\partial \pi_{1}^{*}}{\partial \varphi}>0,{ }^{22}$ which shows that firms 1 and 2's profits increase with $\varphi$. Intuitively a larger $\varphi$ implies more intense competition among other firms, which benefits firms 1 and 2.

\footnotetext{
${ }^{21}$ Note $\frac{\partial q_{1}^{*}}{\partial \delta}=\frac{-(N-3)(\alpha-c)(2+\gamma-2 \varphi) A}{[(2+\gamma) A-2 \varphi B]^{2}}, \frac{\partial p_{1}^{*}}{\partial \delta}=\frac{\partial q_{1}^{*}}{\partial \delta}$ and $\frac{\partial \pi_{1}^{*}}{\partial \delta}=2 q_{1}^{*} \frac{\partial q_{1}^{*}}{\partial \delta}$.

${ }^{22}$ Note $\frac{\partial q_{1}^{*}}{\partial \varphi}=\frac{(\alpha-c)[2 B(A-B)+(N-3) B(2+\gamma-2 \varphi)]}{[(2+\gamma) A-2 \varphi B]^{2}}, \frac{\partial p_{1}^{*}}{\partial \varphi}=\frac{\partial q_{1}^{*}}{\partial \varphi}$ and $\frac{\partial \pi_{1}^{*}}{\partial \varphi}=2 q_{1}^{*} \frac{\partial q_{1}^{*}}{\partial \varphi}$.
} 
Concerning firms other than 1 and 2 we observe firstly that $\frac{\partial q_{i}^{*}}{\partial \gamma}, \frac{\partial p_{i}^{*}}{\partial \gamma}$ and $\frac{\partial \pi_{i}^{*}}{\partial \gamma}>0 .{ }^{23}$ This says their equilibrium outputs, prices and profits increase in $\gamma$. Because of this more intense competition (greater $\gamma$ ) between firms 1 and 2 will make the other firms better off. Secondly, it is easy to see that $\frac{\partial q_{i}^{*}}{\partial \delta}, \frac{\partial p_{i}^{*}}{\partial \delta}$ and $\frac{\partial \pi_{i}^{*}}{\partial \delta}>0$ (because $\delta$ enters only through $B$ in the denominators), which shows that the more competitive pressure firm $i$ imposes on firm 1 (i.e., greater $\delta$ ), the more firm $i$ gains. Finally, we can show that $\frac{\partial q_{i}^{*}}{\partial \varphi}, \frac{\partial p_{i}^{*}}{\partial \varphi}$ and $\frac{\partial \pi_{i}^{*}}{\partial \varphi}<0,{ }^{24}$ which suggests that greater competition among these firms reduces their own profits.

These observations of how equilibrium prices and quantities are affected by substitution parameters largely extend the previous researches. In those researches symmetric substitution is typically assumed and it is not clear how a firm's welfare is affected by different degrees of substitutability between different firms. In this chapter we present a more complete picture about how a firm's output, price and profit change with substitutability between his product and the products either of his potential merging partners or of outsiders, or with substitutability among outsiders' products.

One common observation both in those symmetric models and in our model is the negative relationship between a firm's welfare and the degree of substitution between its product and those of its rivals. In the spatial Bertrand model, a firm's equilibrium price and quantity typically increase in the distance between its and its rival's products. It is so for the simple reason that increased distance implies a lower degree of substitutability

$$
\begin{aligned}
& { }^{23} \text { Note } \frac{\partial q_{i}^{*}}{\partial \gamma}=\frac{2 \varphi(\alpha-c)(A-B)}{[(2+\gamma) A-2 \varphi B]^{2}}, \frac{\partial p_{i}^{*}}{\partial \gamma}=\frac{\partial q_{i}^{*}}{\partial \gamma} \text { and } \frac{\partial \pi_{i}^{*}}{\partial \gamma}=2 q_{i}^{*} \frac{\partial q_{i}^{*}}{\partial \gamma} . \\
& { }^{24} \text { Note } \frac{\partial q_{i}^{*}}{\partial \varphi}=\frac{-2(\alpha-c)(2+\gamma)(A-B)}{[(2+\gamma) A-2 \varphi B]^{2}}, \frac{\partial p_{i}^{*}}{\partial \varphi}=\frac{\partial q_{i}^{*}}{\partial \varphi} \text { and } \frac{\partial \pi_{i}^{*}}{\partial \varphi}=2 q_{i}^{*} \frac{\partial q_{i}^{*}}{\partial \varphi}
\end{aligned}
$$


between two neighboring firms and fewer competitors in a given market (Braid 1999, Levy and Reitzes 1989, Salop 1979). The non-spatial Bertrand model gives similar results that the more the merging products are differentiated, the higher the price and profit are (Deneckere and Davison 1985). The same results are observed in Hsu and Wang (2010) when a Cournot model is employed.

We now proceed to examine the differences in the quantity and price of two different firms in the pre-merger equilibrium.

$$
\begin{aligned}
& \Delta q^{*}=q_{1}^{*}-q_{i}^{*}=\frac{(\alpha-c)[(N-1) \varphi-(N-2) \delta-\gamma]}{(2+\gamma) A-2 \varphi B} \\
& \Delta p^{*}=p_{1}^{*}-p_{i}^{*}=\frac{(\alpha-c)[(N-1) \varphi-(N-2) \delta-\gamma]}{(2+\gamma) A-2 \varphi B}=q_{1}^{*}-q_{i}^{*}
\end{aligned}
$$

Equation (2.17) tells us that $p_{1}^{*} \gtrless p_{i}^{*} \Leftrightarrow q_{1}^{*} \gtrless q_{i}^{*}$, so firm 1 will either have both higher price and output or both lower price and output than firm $i(=3,4, \ldots N)$. Since the terms that determine the sign of both equations, $(N-1) \varphi-(N-2) \delta-\gamma$, could be either positive or negative, so are the signs of $\Delta q^{*}$ and $\Delta p^{*}$. We thereby conclude that $p_{1}^{*}>p_{i}^{*}$ iff $(N-1) \varphi-(N-2) \delta-\gamma>0$. From the latter condition we can see that a larger $\gamma$ (a higher degree of substitutability between firms 1 and 2) will make it more likely that firm 1 will earn less than firm $i$. However, the size of $\gamma$ by itself cannot decide whether firm 1 enjoys higher earnings than firm $i$.

\subsection{Merger Analysis}

\subsubsection{Post-Merger Equilibrium}

When firms 1 and 2 merge, they coordinate output levels to maximize their joint profits. The merged entity's optimization problem is: 


$$
\max _{q_{1}, q_{2}}\left(p_{1}-c\right) q_{1}+\left(p_{2}-c\right) q_{2}
$$

The first order conditions are

$$
\begin{aligned}
& \alpha-2 q_{1}-2 \gamma q_{2}-\delta \sum_{j=3}^{N} q_{j}-c=0 \\
& \alpha-2 \gamma q_{1}-2 q_{2}-\delta \sum_{j=3}^{N} q_{j}-c=0
\end{aligned}
$$

Solving for (2.19) and (2.20) we have

$$
q_{1}=q_{2}=\frac{\alpha-c-\delta \sum_{i=3}^{N} q_{i}}{2(1+\gamma)}
$$

Firm $i$ faces the same optimization problem as before the merger, and hence its first order condition is identical to equation (2.9).

Plugging (2.21) into (2.9) we have the post-merger prices and quantities:

$$
\begin{aligned}
& q_{1}^{m}=q_{2}^{m}=\frac{[2+(N-3) \varphi-(N-2) \delta](\alpha-c)}{2(1+\gamma)[(N-3) \varphi+2]-2(N-2) \varphi \delta}=\frac{(A-B)(\alpha-c)}{2(1+\gamma) A-2 \varphi B} \\
& q_{i}^{m}=\frac{(1+\gamma-\varphi)(\alpha-c)}{(1+\gamma)[(N-3) \varphi+2]-(N-2) \varphi \delta}=\frac{(1+\gamma-\varphi)(\alpha-c)}{(1+\gamma) A-\varphi B} \\
& p_{1}^{m}=p_{2}^{m}=\frac{(\alpha-c)(1+\gamma)(A-B)+c[2(1+\gamma) A-2 \varphi B]}{2(1+\gamma) A-2 \varphi B}=(1+\gamma) q_{1}^{m}+c \\
& p_{i}^{m}=\frac{c(1+\gamma) A-c \varphi B+(\alpha-c)(1+\gamma-\varphi)}{(1+\gamma) A-\varphi B}=q_{i}^{m}+c \\
& \pi_{1}^{m}=\pi_{2}^{m}=\frac{(1+\gamma)(\alpha-c)^{2}(A-B)^{2}}{4[(\beta+\gamma) A-\varphi B]^{2}}=(1+\gamma)\left(q_{1}^{m}\right)^{2} \\
& \pi_{i}^{m}=\frac{(\alpha-c)^{2}(1+\gamma-\varphi)^{2}}{[(1+\gamma) A-\varphi B]^{2}}=\left(q_{i}^{m}\right)^{2}
\end{aligned}
$$

It is easy to verify that the equilibrium prices and quantities are all positive. Comparative statics show that the parameters of demand substitutability have the same effects on prices and quantity as in section 2.2. Specifically, the merging firms' welfare 
decreases in $\gamma$ and $\delta$ since $\frac{\partial q_{1}^{m}}{\partial \gamma}, \frac{\partial p_{1}^{m}}{\partial \gamma}, \frac{\partial \pi_{1}^{m}}{\partial \gamma}<025$ and $\frac{\partial q_{1}^{m}}{\partial \delta}, \frac{\partial p_{1}^{m}}{\partial \delta}, \frac{\partial \pi_{1}^{m}}{\partial \delta}<0,{ }^{26}$ and increases in $\varphi$ since $\frac{\partial q_{1}^{m}}{\partial \varphi}, \frac{\partial p_{1}^{m}}{\partial \varphi}$ and $\frac{\partial \pi_{1}^{m}}{\partial \varphi}>0 .{ }^{27}$ On the other hand, the non-merging firms' welfare increases in $\gamma$ and $\delta$ because $\frac{\partial q_{i}^{m}}{\partial \gamma}, \frac{\partial p_{i}^{m}}{\partial \gamma}, \frac{\partial \pi_{i}^{m}}{\partial \gamma}>0,{ }^{28} \frac{\partial q_{i}^{m}}{\partial \delta}, \frac{\partial p_{i}^{m}}{\partial \delta}, \frac{\partial \pi_{i}^{m}}{\partial \delta}>0^{29}$ and $\frac{\partial q_{i}^{m}}{\partial \varphi}, \frac{\partial p_{i}^{m}}{\partial \varphi}$, $\frac{\partial \pi_{i}^{m}}{\partial \varphi}<0 .^{30}$

As in the pre-merger case, the negative relationship between the insiders' postmerger prices or profits and the degree of product substitution $\gamma$ is also typically observed in the merger literature (Braid 1999, Deneckere and Davison 1985, Hsu and Wang 2010, Levy and Reitzes 1992, Salop 1979). ${ }^{31}$ In our model this negative relationship between $\gamma$ and the insiders' post-merger quantities, prices and profits is always true, except for the prices in a market where duopoly firms merge into a monopoly. ${ }^{32}$ The reason for these results is that quantities are strategic substitutes. When firms 1 and 2 merge, they internalize the negative impact of a larger $q_{1}$ on the profits earned from product 2 (and

${ }^{25} \frac{\partial q_{1}^{m}}{\partial \gamma}<0$ is obvious since $\gamma$ only shows in denominator; $\frac{\partial p_{1}^{m}}{\partial \gamma}<0$ because $p_{1}^{m}=(1+\gamma) q_{1}^{m}+c=$ $\frac{(1+\gamma)(A-B)(\alpha-c)}{2(1+\gamma) A-2 \varphi B}+c=\frac{(A-B)(\alpha-c)}{2 A-\frac{2 \varphi B}{1+\gamma}}+c$ and $\frac{\partial \pi_{1}^{m}}{\partial \gamma}=-\left(q_{1}^{m}\right)^{2} \frac{(1+\gamma) A+\varphi B}{(1+\gamma) A-\varphi B}<0$.

$26 \frac{\partial q_{1}^{m}}{\partial \delta}=\frac{-2(\alpha-c)(N-2) A(1+\gamma-\varphi)}{[2(1+\gamma) A-2 \varphi B]^{2}}<0, \frac{\partial p_{1}^{m}}{\partial \delta}=(1+\gamma) \frac{\partial q_{1}^{M}}{\partial \delta}$ and $\frac{\partial \pi_{1}^{m}}{\partial \delta}=2(1+\gamma) q_{1}^{m} \frac{\partial q_{1}^{m}}{\partial \delta}$.

$27 \frac{\partial q_{1}^{m}}{\partial \varphi}=\frac{(\alpha-c)[2 B(A-B)+2(N-3) B(1+\gamma-\varphi)]}{[2(1+\gamma) A-2 \varphi B]^{2}}>0, \frac{\partial p_{1}^{m}}{\partial \varphi}=\frac{\partial q_{1}^{m}}{\partial \varphi}$ and $\frac{\partial \pi_{1}^{m}}{\partial \varphi}=2(1+\gamma) q_{1}^{m} \frac{\partial q_{1}^{m}}{\partial \varphi}$.

$28 \frac{\partial q_{i}^{m}}{\partial \gamma}=\frac{(\alpha-c) \varphi(A-B)}{[(1+\gamma) A-\varphi B]^{2}}>0, \frac{\partial p_{i}^{m}}{\partial \gamma}=\frac{\partial q_{i}^{M}}{\partial \gamma}, \frac{\partial \pi_{i}^{m}}{\partial \gamma}=2 q_{i}^{m} \frac{\partial q_{i}^{m}}{\partial \gamma}$.

$29 \frac{\partial q_{i}^{m}}{\partial \delta}<0$ is obvious because $\delta$ only shows in $B$ which is in the denominator with negative sign; $\frac{\partial p_{i}^{m}}{\partial \delta}=$ $\frac{\partial q_{i}^{m}}{\partial \delta}, \frac{\partial \pi_{i}^{m}}{\partial \delta}=2 q_{i}^{m} \frac{\partial q_{i}^{m}}{\partial \delta}$.

$30 \frac{\partial q_{i}^{m}}{\partial \varphi}=\frac{-(\alpha-c)(1+\gamma)[(N-1)+(N-3) \gamma-(N-2) \delta]}{[(1+\gamma) A-\varphi B]^{2}}<0, \frac{\partial p_{i}^{m}}{\partial \varphi}=\frac{\partial q_{i}^{m}}{\partial \varphi}, \frac{\partial \pi_{i}^{m}}{\partial \varphi}=2 q_{i}^{m} \frac{\partial q_{i}^{m}}{\partial \varphi}$.

${ }^{31}$ There is an exception about the relationship between the outsiders' post-merger profits and $\gamma$ in a numerical example (Table 1) of Deneckere and Davison (1985). In this example, the insiders' post-merger profits always decrease in $\gamma$ but the outsiders' post-merger profits also decrease in $\gamma$ when the number of firms that merge is less than 8 .

${ }^{32}$ In this case, pre-merger $q_{1}^{*}=\frac{\alpha-c}{2+\gamma}, p_{1}^{*}=\frac{\alpha-c}{2+\gamma}+c, \pi_{1}^{*}=\frac{(\alpha-c)^{2}}{(2+\gamma)^{2}}$ while post-merger $q_{1}^{m}=\frac{\alpha-c}{2(+\gamma)}, p_{1}^{m}=\frac{\alpha+c}{2}$, $\pi_{1}^{m}=\frac{(\alpha-c)^{2}}{4(1+\gamma)}$. The relationship mentioned above is true except when $p_{1}^{m}=\frac{\alpha+c}{2}$. 
vice versa). Consequently, the merger causes them to restrain their quantities. The higher the degree of substitutability between firms 1 and 2, the stronger the incentive to restrain their quantities after the merger. Thus, a greater $\gamma$ leads to smaller outputs and lower profits.

The above analysis gives our first proposition:

Proposition 2.1 The merging firms' quantities, prices and profits decrease in $\gamma$ and $\delta$, and increase in $\varphi$.

\subsubsection{Merger Profitability}

A merger typically raises prices and reduces outputs, so the merger will not be profitable unless the gains due to higher prices overwhelm losses from lower outputs. The merger paradox arises when a merger reduces the merging parties' total profits relative to their combined pre-merger profit levels. In the literature, the merger paradox is usually found in models of quantity competition rather than price competition because quantities are strategic substitutes while prices are strategic complements (Deneckere and Davidson 1985). Since our model is one of quantity competition, we must first examine the conditions for a profitable merger.

As mentioned previously, a merger enables firms 1 and 2 to internalize the negative impact of a larger $q_{1}$ on the profits earned by product 2 (and vice versa). This causes them to restrain their output levels. The merger becomes unprofitable for the two firms if this output contraction is too large. Indeed, we can write the change in firm 1's profit before and after the merger as: $\pi_{1}^{m}-\pi_{1}^{*}=(1+\gamma)\left(q_{1}^{m}\right)^{2}-\left(q_{1}^{*}\right)^{2}$. Then we have the following proposition:

Proposition 2.2 The merger is profitable if and only if $\frac{q_{1}^{m}}{q_{1}^{*}}>\sqrt{\frac{1}{1+\gamma}}$. 
The drawback of Proposition 2.2 is that quantities are endogenous variables. Next we derive the conditions for a profitable merger based on exogenous parameters:

$$
\Delta \pi_{1}=\pi_{1}^{m}-\pi_{1}^{*}=(\alpha-c)^{2}(A-B)^{2} \gamma \frac{(1+\gamma) \gamma A^{2}-4 \varphi(1+\gamma) A B+4 \varphi^{2} B^{2}}{4[(1+\gamma) A-\varphi B]^{2}[(2+\gamma) A-2 \varphi B]^{2}}
$$

Since the denominator of (2.28) is positive, the merger is profitable if the numerator of (2.28) is positive. This requires that

$$
A^{2} \gamma^{2}+\left(A^{2}-4 \varphi A B\right) \gamma-4 \varphi A B+4 \varphi^{2} B^{2}>0
$$

We shall present Proposition 2.3 in terms of $\gamma$, not only because it is the substitution parameter that is our main concern but also because of the convenience that both $A$ and $B$ are independent of $\gamma$.

Proposition 2.3 The merger is profitable if and only if $\frac{4 \varphi B-A+\sqrt{A^{2}+8 \varphi A B}}{2 A}<\gamma<1$.

- Proof: Inequality (2.29) stands for the positive portion of an upward-opening parabola that crosses the horizontal axis at $\gamma_{p 1}, \gamma_{p 2}=\frac{4 \varphi B-A \pm \sqrt{A^{2}+8 \varphi A B}}{2 A}$. Since $|4 \varphi B-A|<$ $\sqrt{A^{2}+8 \varphi A B}$, we have $\gamma_{p 1}<0<\gamma_{p 2}$. Thus, the merger is profitable if and only if $\frac{4 \varphi B-A+\sqrt{A^{2}+8 \varphi A B}}{2 A}<\gamma<1$

Proposition 2.3 is not a reader-friendly illustration of the profitability conditions. To make it more readable, we wish to develop a diagrammatic illustration of the profitability conditions in the form of a relationship between substitutability parameters, and a plot of $\gamma$ with respect to $\delta$ would thus seem the best practicable choice. Note that the profitability condition (2.29) is just the area above the upper contour of the corresponding equation

$$
A^{2} \gamma^{2}+\left(A^{2}-4 \varphi A B\right) \gamma+4 \varphi^{2} B^{2}-4 \varphi A B=0
$$


Equation (2.30) implicitly defines a function $\gamma(\delta)$. Figure 2.1 depicts $\gamma$ in terms of $\delta$ and the shady area is the profitable area.

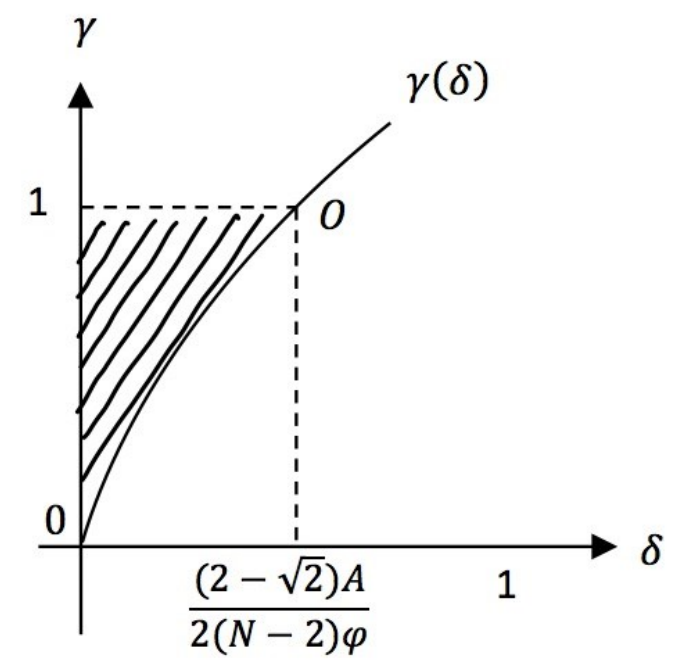

Figure 2.1 Profitable Mergers

Lemma 2.1 The function $\gamma(\delta)$ is concave and monotonically increasing in $\delta$.

-Proof:

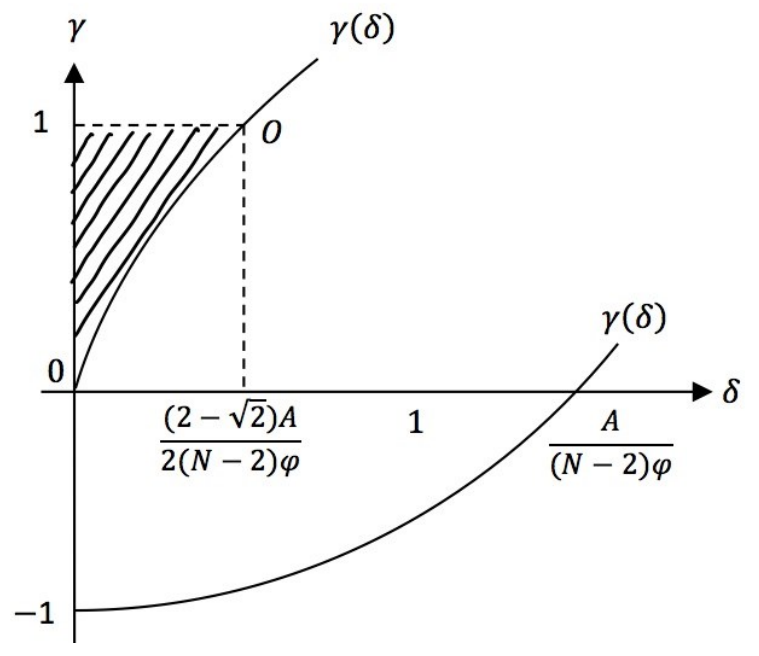

Figure 2.2 Function $\gamma(\delta)$

The solution to equation (2.30) is $\gamma=\frac{4 \varphi B-A \pm \sqrt{A^{2}+8 \varphi A B}}{2 A}$. It is easy to show that $|4 \varphi B-A|<\sqrt{A^{2}+8 \varphi A B}$, from which we know that $\gamma$ always has two values, one 
positive and one negative, for each value of $\delta>0$ (or $B>0$, when our general assumptions apply). The positivity of $\gamma$ would thus suggest that there would be a one to one mapping between $\gamma$ and $\delta$, and $0 O$ is the only relevant $\gamma(\delta)$ curve. $^{33}$

We now proceed to check the concavity of $\gamma(\delta)$. Since only the upper contour $0 O^{\prime}$ is considered, the value of $\gamma(\delta)$ is given by $\frac{4 \varphi B-A+\sqrt{A^{2}+8 \varphi A B}}{2 A}$. Now it is immediate that $\frac{\partial \gamma}{\partial \delta}>0$, and $\frac{\partial}{\partial \delta}\left(\frac{\partial \gamma}{\partial \delta}\right)<0$ so the curve is upward sloping and concave.

Based on Figure 2.1, we are able to find sufficient conditions for merger profitability.

Proposition 2.4 A sufficient condition for merger profitability is $\frac{\gamma}{\delta} \geq \frac{4(N-2) \varphi}{A}$.

-Proof:

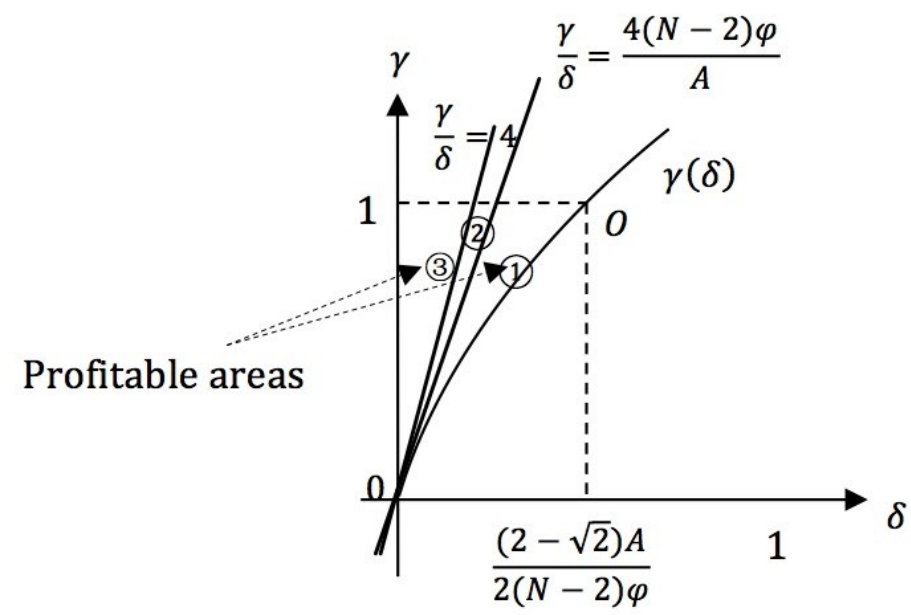

Figure 2.3 A Sufficient Condition for Profitable Mergers

\footnotetext{
${ }^{33}$ This conclusion and that $|4 \varphi B-A|<\sqrt{A^{2}+8 \varphi A B}$ is drawn under the assumption max $\{\gamma, \delta, \varphi\}<1$. For the general case of (2.30) we would find two $\gamma(\delta)$ curves both in the first quadrant (the lower curve in Figure 2.2 will finally cross the horizontal axis and rise upward), meaning that there are two positive $\gamma$ values for each positive $\delta$, but that is only because the assumption $\max \{\gamma, \delta, \varphi\}<1$ is violated.
} 
$\left.\frac{\partial \gamma}{\partial \delta}\right|_{\gamma=0, \delta=0}=\left.\frac{\partial \gamma}{\partial B} \frac{\partial B}{\partial \delta}\right|_{\gamma=0, \delta=0}=\frac{4(N-2) \varphi}{A}$, and because of concavity of $\gamma(\delta)$, any value of $\gamma \geq \frac{4(N-2) \varphi \delta}{A}$ will make the merger profitable.

Proposition 2.4 states that the merger is profitable if the ratio $\gamma / \delta$ is sufficiently large. It is easy to verify that $4>\frac{4(N-2) \varphi}{A}$. Hence the condition in Proposition 2.4 can be satisfied by $\gamma \geq 4 \delta .{ }^{34}$ In Figure 2.3 above, the mergers are profitable in all three regions numbered (1), (2) and (3), and $\gamma \geq \frac{4(N-2) \varphi \delta}{A}$ corresponds to (2) and (3), while $\gamma \geq 4 \delta$ corresponds to (3).

We could depict the implicit function $\gamma(\delta)$ of (2.30) by assigning each parameter a value. For example, if we assume $N=10, \varphi=0.5$, then equation (2.30) would become $30.25 \gamma^{2}+30.25 \gamma-88 \gamma \delta+64 \delta^{2}-88 \delta=0$, which is illustrated in Figure 2.4. Note in Figure 2.4 that $\gamma$ is on the vertical axis and $\delta$ on the horizontal axis. Since the relevant values of $\gamma$ and $\delta$ are between 0 and 1 , only the portion of the curve above the $(0,0)$ point in Figure 2.4 is relevant for our result.

\footnotetext{
${ }^{34}$ It is also easy to show that $\gamma>2 \delta$ is a sufficient condition for merger profitability when $N=3$. Ebina and Shimizu (2009) show that if $\gamma<0.307$, then the condition $\gamma>\delta$ (in our notation) guarantees merger profitability. Their model however is simplified in substitution relations and their result is a special case.
} 


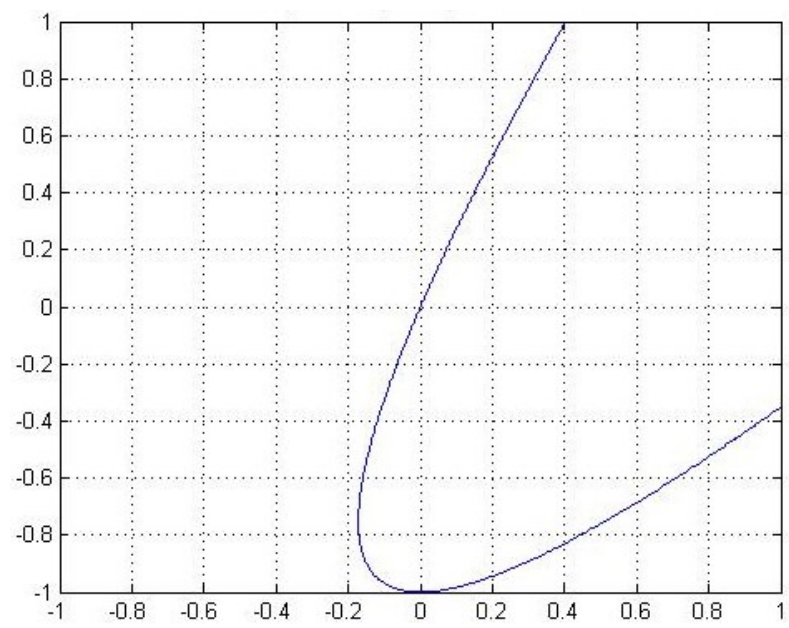

Figure 2.4 Function $\gamma(\delta)$ when $N=10, \varphi=0.5$

Proposition 2.3 states that $\gamma$ has to be sufficiently large in order for the merger be profitable. It is interesting to contrast this result with Proposition 2.1, which says that the merged entity's profits are actually lower if $\gamma$ becomes larger. The reason for these seemingly inconsistent results is that the pre-merger profits are also lower when $\gamma$ becomes larger. Merger profitability, on the other hand, depends on the difference between post-merger and pre-merger profits. The latter is positive if $\gamma$ is sufficiently large (relative to $\delta) .{ }^{35}$

Our finding is in sharp contrast to the result in Hsu and Wang (2010) that a merger is profitable only when there is a sufficiently low degree of substitutability between the merging products. In their model, however, $\gamma=\delta=\varphi$, which means that all firms have the same degree of substitutability. Consequently, a low $\gamma$ also implies a low $\delta$; in other words, a low degree of substitutability between the merging products also implies a low

\footnotetext{
${ }^{35}$ Some different observations are found in several articles. Deneckere and Davison (1985) and M'Chirgui and Hichri (2006) show that a merger is profitable when the substitutability between the merging products is at an intermediate level. The former use a numerical example with linear demand and the latter is based on a downstream duopolistic merger. Perry and Porter (1985) observe that a merger is profitable when conjectural variation, which is also an indicator of the degree of competition, is at an intermediate level in an oligopoly market with a larger number of dominant firms.
} 
degree of substitutability between an insider's and an outsider's products. Our analysis suggests that it is the low $\delta$ that drives the result in Hsu and Wang (2010).

Next, we examine how the degree of merger profitability, as measured by $\Delta \pi_{1}$, is affected by various parameters in this model. We start with $\gamma$, the degree of substitutability between the merging firms $(\gamma)$. We already know that a profitable merger requires that the merging goods be sufficiently close substitutes, and we will further prove that merging firms' profitability increases in $\gamma$ and that the greatest profitability occurs when $\gamma$ approaches 1 .

Proposition 2.5 Merger profitability increases in $\gamma$. In other words, mergers are more profitable when the degree of substitutability between the merging products is higher.

- Proof: Differentiating (2.28) with respect to $\gamma$ (note we only consider the region of price increase), we have

$\frac{\partial \Delta \pi_{1}}{\partial \gamma}=(\alpha-c)^{2}(A-B)^{2} \frac{-A^{4} \gamma^{4}+A^{3} \gamma^{3}(z+6 \varphi B)+6 A^{2} \gamma^{2} z(z+2 \varphi B)+4 A \gamma z^{3}-8 \varphi B z^{3}}{4(\gamma A+z)^{3}(\gamma A+2 z)^{3}}$

where $z=A-\varphi B$. The sign of (2.31) is determined by

$$
\overbrace{-A^{4} \gamma^{4}+A^{3} \gamma^{3}(z+6 \varphi B)+6 A^{2} \gamma^{2} z(z+2 \varphi B)}^{(2.32 .1)}+\overbrace{4 z^{3}(A \gamma-2 \varphi B)}^{(2.32 .2)} \text {. }
$$




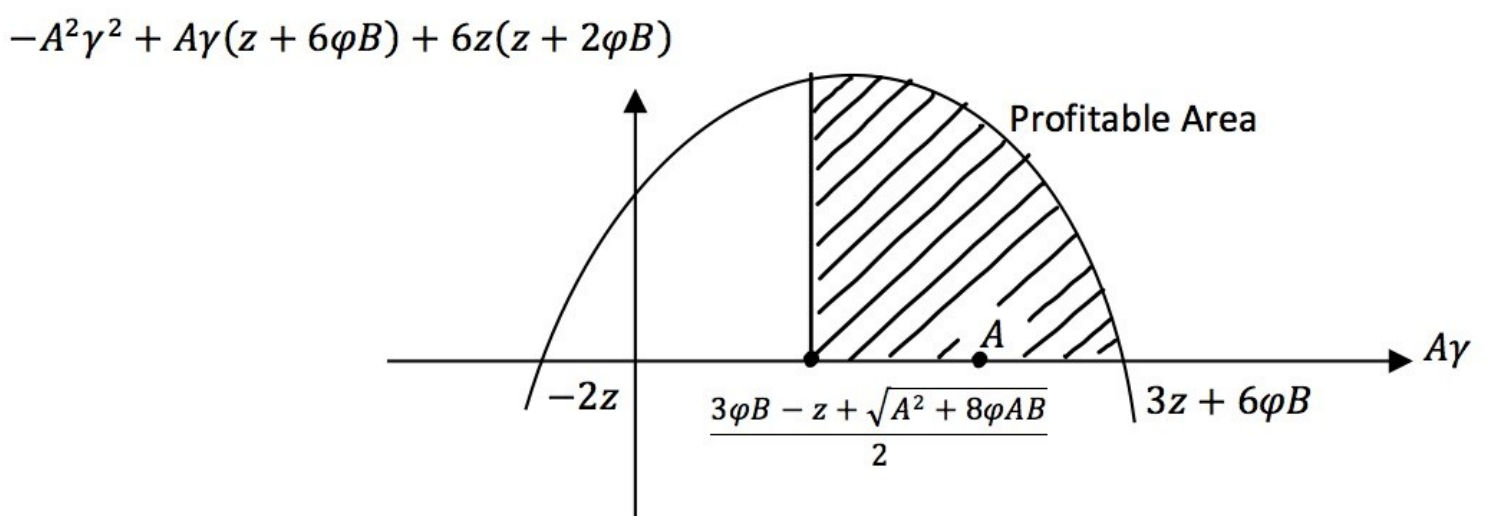

Figure 2.5 Function $-A^{2} \gamma^{2}+A \gamma(z+6 \varphi B)+6 z(z+2 \varphi B)$

We divide (2.32) into two parts, (2.32.1) and (2.32.2), for convenience. The terms in (2.32.2) are positive because the condition for a profitable merger implies that $A \gamma>$ $2 \varphi B ;{ }^{36}$ the terms in $(2.32 .1)$ can be rearranged as $A^{2} \gamma^{2}\left[-A^{2} \gamma^{2}+A \gamma(z+6 \varphi B)+\right.$ $6 z(z+2 \varphi B)]$, with the terms in the brackets, $-A^{2} \gamma^{2}+A \gamma(z+6 \varphi B)+6 z(z+2 \varphi B)$, being a downward-opening parabola that crosses the horizontal axis at $-2 z$ and $3 z+$ $6 \varphi B$ (see Figure 2.5).

It is immediate that $A \gamma<A<3 z+6 \varphi B$, thus making (2.31) positive. Note that the condition for a profitable merger, $\gamma>\frac{4 \varphi B-A+\sqrt{A^{2}+8 \varphi B}}{2 A}$, can be rewritten as $A \gamma>$ $\frac{3 \varphi B-z+\sqrt{A^{2}+8 \varphi A B}}{2}$.

Next, we consider how $N$, the number of firms in the market, affects merger profitability. Intuitively a merger should be more profitable when there are fewer firms in the market. $^{37}$

${ }^{36}$ Proposition 2.3 shows that $\gamma>\frac{4 \varphi B-A+\sqrt{A^{2}+8 \varphi A B}}{2 A} \Leftrightarrow 2 A \gamma>4 \varphi B-A+\sqrt{A^{2}+8 \varphi A B}>4 \varphi B$.

${ }^{37}$ When $N=2$ (which we assume away) the merger would always be profitable, i.e., (2.29) will always hold. However, this is not true for $N \geq 3$. 
Proposition 2.6 There exists a $N^{*}$ below which the merger would be profitable. Furthermore, a greater $\gamma$ broadens the range of $N$ for which the merger is profitable, and a smaller $N$ broadens the range of $\gamma$ for profitable mergers.

- Proof: Condition (2.29) can be rewritten as $\left(\frac{A}{B}\right)^{2} \gamma(1+\gamma)-4 \varphi(1+\gamma)\left(\frac{A}{B}\right)+4 \varphi^{2}>$ 0 . The roots of the corresponding equation are $\frac{A}{B}=\frac{2 \varphi}{\gamma}\left(1 \pm \sqrt{\frac{1}{1+\gamma}}\right)$. Since $\frac{A}{B}>1$, it must be greater than $K \equiv \frac{2 \varphi}{\gamma}\left(1+\sqrt{\frac{1}{1+\gamma}}\right)$ to satisfy inequality (2.29). ${ }^{38}$ Since $\frac{A}{B}=\frac{(N-3) \varphi+2}{(N-2) \delta}$, there must exist a critical $N^{*}=\left\lfloor\frac{2+2 \delta K-3 \varphi}{\delta K-\varphi}\right\rfloor$ which equates $\frac{A}{B}$ and $K$. Because $\frac{\partial\left(\frac{A}{B}\right)}{\partial N}=$ $\frac{\partial\left[\frac{(N-3) \varphi+2}{(N-2) \delta}\right]}{\partial N}=(\varphi-2) \delta<0$, any $N<N^{*}$ will make $\frac{A}{B}>\frac{2 \varphi}{\gamma}\left(1+\sqrt{\frac{1}{1+\gamma}}\right)$, in which case the merger is profitable.

Regarding how $\gamma$ affects $N^{*}$, note that $K$ clearly decreases in $\gamma$, so a greater $\gamma$ would reduce the threshold of $\frac{A}{B}$ for merger profitability. Since $\frac{A}{B}$ decreases in $N$, a lower threshold of $\frac{A}{B}$ would thus imply greater $N^{*}$. Hence, a greater $\gamma$ broadens the range of $N$ for which the merger is profitable.

${ }^{38}$ This is because $\frac{A}{B}>\frac{2 \varphi}{\gamma}$. Please refer to Note 36 . 


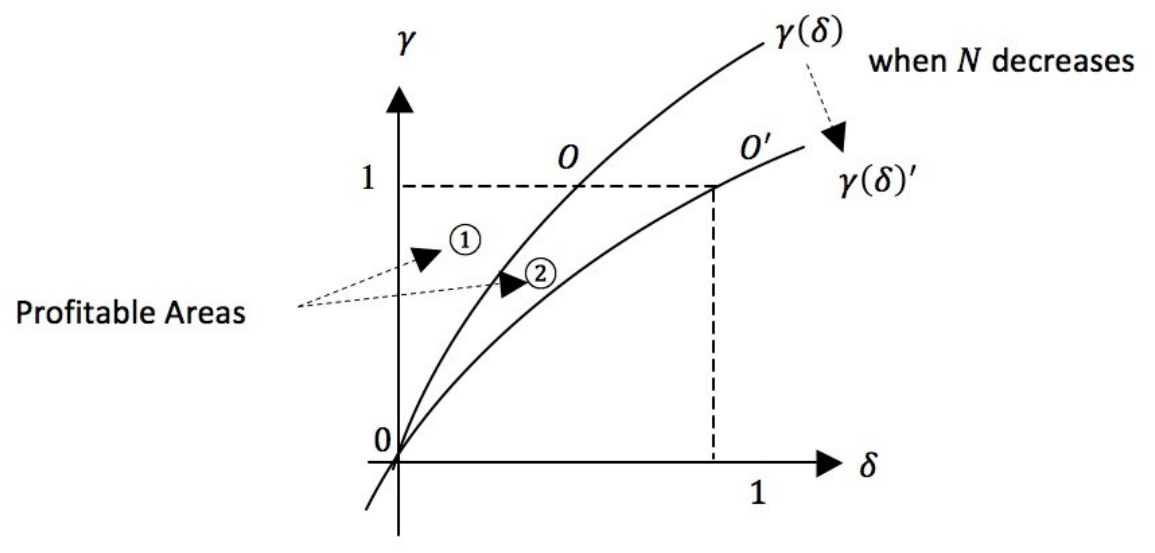

Figure 2.6 How $N$ Affects Merger Profitability

Figure 2.6 also helps to illustrate how $N$ affects merger profitability. Recall that in Figure 2.3 the slope of the curve $\gamma(\delta)$ at the origin $\left.\frac{\partial \gamma}{\partial \delta}\right|_{\gamma=0, \delta=0}$ is $\frac{4(N-2) \varphi}{A}$, which could be easily shown to increase in $N$. Actually $\frac{\partial \gamma}{\partial \delta}=\frac{2 \varphi}{\delta} \cdot \frac{B}{A}\left(1+\frac{1}{\sqrt{1+8 \varphi\left(\frac{B}{A}\right)}}\right)$ and we could show that $\frac{\partial \gamma}{\partial \delta}$ increases in $\frac{B}{A}$. With that $\frac{A}{B}$ decreases in $N$, we conclude that $\frac{\partial \gamma}{\partial \delta}$ increases in $N$. As a result, a smaller $N$ would reduce $\frac{\partial \gamma}{\partial \delta}$ and therefore spin the curve $\gamma(\delta)$ downward. This broadens the profitability area from (1) to (1)+(2). In other words, a smaller $N$ broadens the range of $\gamma$ for merger profitability.

Proposition 2.6 shows that unilateral effects analysis is in part structural: sufficient level of market concentration could always make merger profitable (and thus priceincreasing). With higher degree of concentration merging products could be more different while still making merger profitable; conversely when the degree of substitutability between merging products is higher, merger is more profitable and the market could thus accommodate more firms. 
Finally, we investigate how merger profitability is affected by $\delta$, the degree of substitutability between the products of an insider and an outsider to the merger.

Proposition 2.7 There exists a $\delta^{*}$ below which the merger would be profitable. Furthermore, a greater $\gamma$ broadens the range of $\delta$ for which the merger is profitable.

- Proof: From the proof of Proposition 2.6 we learn that the profitability condition can be written as $\frac{A}{B}>\frac{2 \varphi}{\gamma}\left(1+\sqrt{\frac{1}{1+\gamma}}\right)$. Therefore, $\delta$ must be less than $\delta^{*}=A /[(N-$ 2) $\left.\frac{2 \varphi}{\gamma}\left(1+\sqrt{\frac{1}{1+\gamma}}\right)\right]$ for a merger to be profitable. It is immediate that $\delta^{*}$ increases in $\gamma$. Thus, a larger $\gamma$ will push up the threshold $\delta^{*}$ and broaden the range of $\delta$ under which the merger is profitable.

\subsubsection{Effect of Merger on Quantities}

In this section we study the effects of the merger on the output levels and prices. We do so under the assumption that the condition for a profitable merger is satisfied. Since the two firms would have no incentives to enter into an unprofitable merger, it would not be meaningful to analyze the price and quantity effects of unprofitable mergers.

Proposition 2.8 The merger causes the merging firms to reduce their output and the nonmerging firms to increase their output. A higher degree of substitutability between the merging firms leads to a greater reduction in output by the former and a greater expansion of output by the latter. The aggregate industry output shrinks after the merger. 
- Proof. Note that $q_{1}^{m}=q_{2}^{m}=\frac{(A-B)(\alpha-c)}{2(1+\gamma) A-2 \varphi B}$ and $q_{1}^{*}=q_{2}^{*}=\frac{(A-B)(\alpha-c)}{(2+\gamma) A-2 \varphi B}$. It is clear that the former is less than the latter. Moreover, it can be derived that

$$
\frac{\partial\left|q_{1}^{m}-q_{1}^{*}\right|}{\partial \gamma}=\frac{A(A-B)(\alpha-c)\left(2 z^{2}-\gamma^{2} A^{2}\right)}{2(\gamma A+z)^{2}(\gamma A+2 z)^{2}}
$$

where $z=A-\varphi B$. To show that $\frac{\partial\left|q_{1}^{m}-q_{1}^{*}\right|}{\partial \gamma}>0$, note that $2 z^{2}-\gamma^{2} A^{2}=2 A^{2}+2 \varphi^{2} B^{2}-$ $4 \varphi A B-\gamma^{2} A^{2}=A^{2}+2 \varphi^{2} B^{2}-4 \varphi A B+\left(1-\gamma^{2}\right) A^{2}$. The terms $A^{2}-4 \varphi A B+2 \varphi^{2} B^{2}$ cross the horizontal axis at $\frac{A}{B}=2 \varphi\left(1 \pm \frac{1}{\sqrt{2}}\right)$, while the positive profit condition (2.28) requires $\frac{A}{B}>\frac{2 \varphi}{\gamma}\left(1+\frac{1}{\sqrt{1+\gamma}}\right)$. It is obvious that $2 \varphi\left(1+\frac{1}{\sqrt{2}}\right)<\frac{2 \varphi}{\gamma}\left(1+\frac{1}{\sqrt{1+\gamma}}\right)$, which means that when the merger's profitability condition is met, $A^{2}+2 \varphi^{2} B^{2}-4 \varphi A B$ and thus (2.33) is always positive.

As for the sign of $\frac{\partial\left|q_{1}^{m}-q_{1}^{*}\right|}{\partial \delta}$, note that

$$
\begin{aligned}
& \frac{\partial\left|q_{1}^{m}-q_{1}^{*}\right|}{\partial \delta}=\frac{\partial\left|q_{1}^{m}-q_{1}^{*}\right|}{\partial\left(\frac{A}{B}\right)} \frac{\partial\left(\frac{A}{B}\right)}{\partial B} \frac{\partial B}{\partial \delta} \\
= & \frac{(\alpha-c) \gamma A B^{2}(N-2)}{2(\gamma A+z)^{2}(\gamma A+2 z)^{2}} *\left\{[\varphi(4+3 \gamma)-(1+\gamma)(2+\gamma)]\left(\frac{A}{B}\right)^{2}-4 \varphi^{2}\left(\frac{A}{B}\right)+2 \varphi^{2}\right\} .
\end{aligned}
$$

Unfortunately, the sign is undetermined for the reason that the direction of opening of the parabola $[(1+\gamma)(2+\gamma)-\varphi(4+3 \gamma)]\left(\frac{A}{B}\right)^{2}+4 \varphi^{2}\left(\frac{A}{B}\right)-2 \varphi^{2}$ is not determined. So the impact of $\delta$ on the merging firms' outputs is ambiguous.

For outsiders, $q_{i}^{m}-q_{i}^{*}=(\alpha-c) \frac{(\alpha-c) \varphi \gamma(A-B)}{[(1+\gamma) A-\varphi B][(2+\gamma) A-2 \varphi B]}>0$, which says that the merger causes firm $i$ to expand output. Moreover,

$\frac{\partial\left(q_{i}^{m}-q_{i}^{*}\right)}{\partial \gamma}=\frac{\varphi(A-B)(\alpha-c)\left(2 z^{2}-\gamma^{2} A^{2}\right)}{(\gamma A+z)^{2}(\gamma A+2 z)^{2}}>0$

and 
$\frac{\partial\left(q_{i}^{m}-q_{i}^{*}\right)}{\partial \delta}=\frac{\varphi \gamma(\alpha-c)(N-2) B^{2}}{(\gamma A+z)^{2}(\gamma A+2 z)^{2}}\left\{[\varphi(4+3 \gamma)-(1+\gamma)(2+\gamma)]\left(\frac{A}{B}\right)^{2}-4 \varphi^{2}\left(\frac{A}{B}\right)+2 \varphi^{2}\right\}$.

As in the case of $\frac{\partial\left|q_{1}^{m}-q_{1}^{*}\right|}{\partial \delta}$, we are not able to determine the sign of $\frac{\partial\left(q_{i}^{m}-q_{i}^{*}\right)}{\partial \delta}$. But it is clear that the sign of $\frac{\partial\left|q_{1}^{m}-q_{1}^{*}\right|}{\partial \delta}$ is the same as $\frac{\partial\left(q_{i}^{m}-q_{i}^{*}\right)}{\partial \delta}$. Also it is fairly straightforward to show that $\frac{\partial\left|q_{1}^{m}-q_{1}^{*}\right|}{\partial \gamma}>\frac{\partial\left(q_{i}^{m}-q_{i}^{*}\right)}{\partial \gamma}$ and $\frac{\partial\left|q_{1}^{m}-q_{1}^{*}\right|}{\partial \delta}>\frac{\partial\left(q_{i}^{m}-q_{i}^{*}\right)}{\partial \delta}$, suggesting that the output reaction of a merging firm in response to a change in $\gamma$ or $\delta$ is greater than that of a nonmerging firm.

The change in aggregate industry output is given by $2 q_{1}^{m}+(N-2) q_{i}^{m}-2 q_{1}^{*}-$ $(N-2) q_{i}^{*}=-(\alpha-c) \gamma \frac{(A-B)[A-(N-2) \varphi]}{[(1+\gamma) A-\varphi B][(2+\gamma) A-2 \varphi B]}<0$.

In addition, it is easy to show that $\frac{\partial\left(\frac{q_{1}^{m}}{q_{1}^{*}}\right)}{\partial \delta}<0$ and $\frac{\partial\left(\frac{q_{1}^{m}}{q_{1}^{*}}\right)}{\partial \varphi}<0$; so when the merging firms face more competitive rivals (greater $\delta$ ) or when these rivals engage in more intense competition (greater $\varphi$ ), the merging firms' relative output falls.

\subsubsection{Effect of Merger on Prices}

\subsubsection{Prices of Merging Firms}

It can be derived that

$$
\begin{aligned}
& \Delta p_{1}=p_{1}^{m}-p_{1}^{*}=\frac{(\alpha+c)(1+\gamma) A-(\alpha-c)(1+\gamma) B-2 c \varphi B}{2(1+\gamma) A-2 \varphi B}-\frac{(\alpha+c+c \gamma) A-(\alpha-c+2 \varphi c) B}{(2+\gamma) A-2 \varphi B} \\
& =\frac{\gamma(\alpha-c)(A-B)[(1+\gamma) A-2 \varphi B]}{[2(1+\gamma) A-2 \varphi B][(2+\gamma) A-2 \varphi B]}
\end{aligned}
$$

Thus, the prices of the merged entity will increase if and only if

$$
(1+\gamma) A-2 \varphi B>0
$$

For our purposes we are concerned mainly with the role of $\gamma$ and $\delta$ in deciding our price condition. 
Condition (2.35) suggests that a merger does not necessarily lead to higher prices for the merging firms, if we allow for the possibility of unprofitable mergers. However, since only profitable mergers would be proposed, we focus on profitable mergers. Note that $\frac{A}{B}>\max \left\{1, \frac{2 \varphi}{1+\gamma}\right\}$. If $\frac{2 \varphi}{1+\gamma}<1$ then $\frac{A}{B}>1$ is the condition of price-increase. In other words, as long as pre-merger equilibrium exists, the merger will lead to higher prices if $\gamma>2 \varphi-1$. If $\frac{2 \varphi}{1+\gamma}>1$ then $\frac{A}{B}>\frac{2 \varphi}{1+\gamma}$ is the condition for price-increase.

Proposition 2.9 A profitable merger always leads to higher prices for the merging firms.

-Proof: When $N=3, A=2, B=\delta$, and $\frac{2 \varphi B-A}{A}<0$, therefore $\gamma>\frac{2 \varphi B-A}{A}$ is always true. In the case $N \geq 4$, it can be shown, by referring to Proposition 2.3, that $\frac{4 \varphi B-A+\sqrt{A^{2}+8 \varphi A B}}{2 A}>\frac{2 \varphi B-A}{A}$, which implies that insiders' prices always go up as long as the merger is profitable.

We can also relate the change in the merging firms' prices to the change in their quantities.

Proposition 2.10 The merger leads to higher prices for the merging firms if and only if

$$
\frac{q_{1}^{m}}{q_{1}^{*}}>\frac{1}{1+\gamma} .
$$

Proposition 2.10 says that for prices to increase, the post-merger equilibrium outputs cannot shrink too much. Intuitively, the insiders' output reduction is accompanied by the outsiders' output expansion. These two forces have opposing effects on the prices of the merging firms. If the output reduction by the merging firms is very large, the first effect should dominate the second effect, and prices would fall. 
Since $\sqrt{\frac{1}{1+\gamma}}>\frac{1}{1+\gamma}$, a comparison of Propositions 2.10 and 2.2 confirm that the insiders' prices inevitably increase as long as the merger is profitable. When equilibrium outputs decrease too much, the merger would not be profitable.

Next, we investigate how the magnitude of increases in the insiders' prices is affected by the degree of product substitutability. The existing studies of price competition models suggest that the increase in prices for the merging firms should be larger when they produce closer substitutes. Here we want to see whether the same result holds when firms compete in quantities.

Proposition 2.11 The higher the degree of substitutability between the merging firms, the greater the increase in their prices in both level and percentage terms, i.e., $\frac{\partial \Delta p_{1}}{\partial \gamma}$ and $\frac{\partial\left(\frac{\Delta p_{1}}{p_{1}^{*}}\right)}{\partial \gamma}$ are strictly positive.

-Proof: $\Delta p_{1}=\frac{\gamma(\alpha-c)(A-B)[(1+\gamma) A-2 \varphi B]}{[2(1+\gamma) A-2 \varphi B][(2+\gamma) A-2 \varphi B]}=\frac{\gamma(\alpha-c)(A-B) P}{[P+(1+\gamma) A][P+A]}$ where $P=(1+\gamma) A-2 \varphi B$.

This transformation not only simplifies the derivation but also helps us to focus on situations of profitable mergers where the prices always increase, i.e., $P>0$.

The derivative of $p_{1}^{m}-p_{1}^{*}$ with respect to $\gamma$ is

$$
\frac{\partial \Delta p_{1}}{\partial \gamma}=(\alpha-c)(A-B) \frac{P^{3}+P^{2}(2-\gamma) A+P A^{2}+\gamma(1+\gamma) A^{3}}{[P+(1+\gamma) A]^{2}[P+A]^{2}}>0 \text { if } P>0 .
$$

Moreover,

$$
\begin{aligned}
& \frac{\Delta p_{1}}{p_{1}^{*}}=\frac{\gamma(\alpha-c)(A-B) P}{[P+(1+\gamma) A][c P+\alpha A-(\alpha-c) B]} \\
& \frac{\partial\left(\frac{\Delta p_{1}}{p_{1}^{*}}\right)}{\partial \gamma}=(\alpha-c)(A-B) \frac{c P^{3}+P^{2}[c(1-\gamma) A+J]+P A J+\gamma(1+\gamma) A^{2} J}{[P+(1+\gamma) A]^{2}[c P+\alpha A-(\alpha-c) B]^{2}}>0
\end{aligned}
$$

where $J=\alpha A-(\alpha-c) B>0$. 
Proposition 2.11 confirms that, as in price competition models, the price effect of a merger is larger when the merging firms produce closer substitutes. It demonstrates a different role of $\gamma$ from that in Proposition 2.1. While a small $\gamma$ encourages high pre-andpost-merger prices, it constrains the post-merger price increase in both absolute and relative terms.

Turning to substitutability between insiders and outsiders, note that a larger $\delta$ implies that the non-merging firms impose a stronger competitive pressure on the merging firms. Accordingly, we expect that a larger $\delta$ would mitigate the price increases by the merging firms.

Proposition 2.12 The higher the degree of substitutability between the merging firms' and the non-merging firms' products, the smaller the increases in the prices of the merging firms in both level and percentage terms, i.e., $\frac{\partial \Delta p_{1}}{\partial \delta}$ and $\frac{\partial\left(\frac{\Delta p_{1}}{p_{1}^{*}}\right)}{\partial \delta}$ are strictly negative.

-Proof: From the proof of Proposition 2.11, we have $\Delta p_{1}=\frac{\gamma(\alpha-c)(A-B) P}{[P+(1+\gamma) A][P+A]}$ where $P=(1+\gamma) A-2 \varphi B$. Since $\delta$ only appears in $B$, we only need to take the derivative with respect to $B$. Given that $P>0$

$$
\frac{\partial \Delta p_{1}}{\partial B}=\gamma(\alpha-c) \frac{-P^{3}-P^{2}[(2-2 \varphi+\gamma) A+2 \varphi B]-P(1+\gamma) A^{2}-2 \varphi(1+\gamma)(A-B) A^{2}}{[P+(1+\gamma) A]^{2}[P+A]^{2}}<0,
$$

we have $\frac{\partial \Delta p_{1}}{\partial \delta}=(N-2) \frac{\partial \Delta p_{1}}{\partial B}<0$. Moreover,

$$
\begin{aligned}
& \frac{\partial\left(\frac{\Delta p_{1}}{p_{1}^{*}}\right)}{\partial B}=\gamma(\alpha-c) \frac{-c P^{3}-P^{2}[(2-2 \varphi+\gamma) A+2(c \varphi+\alpha-c) B]-P c(1+\gamma) A^{2}-2 \varphi(1+\gamma)(A-B) A K}{[P+(1+\gamma) A]^{2}[c P+\alpha A-(\alpha-c) B]^{2}}<0, \\
& \frac{\partial\left(\frac{\Delta p_{1}}{p_{1}^{*}}\right)}{\partial \delta}=(N-2) \frac{\partial\left(\frac{\Delta p_{1}}{p_{1}^{*}}\right)}{\partial B}<0 .
\end{aligned}
$$




\subsubsection{Non-Merging Firms Price Change}

The change in the prices of non-merging firms can be written as

$$
\begin{aligned}
& p_{i}^{m}-p_{i}^{*}=\frac{c(1+\gamma) A-c \varphi B+(\alpha-c)(1+\gamma-\varphi)}{(1+\gamma) A-\varphi B}-\frac{c(2+\gamma) A-2 \varphi c B+(\alpha-c)(2+\gamma-2 \varphi)}{(2+\gamma) A-2 \varphi B} \\
& =\frac{\varphi \gamma(\alpha-c)(A-B)}{[(1+\gamma) A-\varphi B][(2+\gamma) A-2 \varphi B]}>0 .
\end{aligned}
$$

This indicates that the merger between two firms causes the prices of all other firms to rise. Note that this is true independent of the substitutability parameters. Combined with the fact that non-merging firms expand outputs we conclude that non-merging firms always increase their profits as long as merger is privately profitable.

To sum up, a profitable merger between firms 1 and 2 causes the merging firms to produce less and non-merging firms to produce more. It also causes the prices of all firms to go up. Since consumer surplus decreases with prices, these results imply that such a merger reduces consumer welfare. Concerning total surplus, as the merger generates no efficiency while the profit-increases for all firms are mere transfers from consumer surplus, it follows that the merger decreases total surplus.

\subsection{Conclusions}

We have studied the effects of merger in a model where firms produce differentiated products and compete in quantities. Different from most of the existing studies of mergers with quantity competition, our model does not involve rising marginal cost, Stackelberg leadership, or entry to study merger profitability. In such a model, the merger paradox would arise if the firms produced a homogeneous product. However, our analysis has shown that product differentiation broadens the range of parameters over which a merger is profitable. In particular, a merger of two firms is profitable if the 
degree of substitutability between the merging firms is sufficiently large relative to that between the merging firms and non-merging firms. A sufficient condition for this to occur is $\gamma / \delta \geq 4$, independent of the number of competitors in the market. Regarding the latter, the merger is profitable as long as the number of competitors is below a certain threshold, and this threshold is raised if the degree of substitutability between the merging firms becomes higher. Moreover, a higher degree of substitutability between the merging firms also expands the range of $\delta$ over which the merger is profitable.

In our model, a profitable merger raises the prices of all firms. In particular, the increase in the prices of the merging firms is larger if their degree of substitutability is higher, or if the degree of substitutability between their products and those of the nonmerging firms is lower. Therefore, the merger reduces consumer surplus, and the harm to consumers is larger if the merging firms produce closer substitutes or if their products are more distant substitutes for the products of other rivals. 


\section{Chapter 3: Merger Efficiencies in a Cournot Model with}

\section{Differentiated Products}

\subsection{Introduction}

Mergers can create efficiencies from sources such as scale economy, rationalized production schedule between plants, and acquirement of complementary technologies. It is commonly believed that the lower costs and/or higher quality brought about by merger efficiencies would encourage firms to compete more aggressively, thus offsetting any loss of competition that may be caused by a merger. As Werden (1996, p.409) has noted, "If a merger caused a reduction in marginal cost for the merging firms, the cost reduction would offset the anticompetitive effect of the merger on prices... Indeed, if the merger reduced the marginal costs of the merging firms by a sufficient amount, it would cause all prices in the industry to fall." Moreover, Williamson (1968) has argued that efficiency gains usually outweigh the losses from increased market power.

In antitrust analysis of mergers, it is common to associate the degree of price reduction with the amount of efficiencies. For example, the 2010 Guidelines (Section 10, pp.30-31) indicate that "the Agencies consider whether cognizable efficiencies likely would be sufficient to reverse the merger's potential to harm customers in the relevant market, e.g., by preventing price increases in that market”. The EC Merger Guidelines (2005, Article 79) states, "The relevant benchmark in assessing efficiency claims is that consumers will not be worse off as a result of the merger. For that purpose, efficiencies should be substantial..." In fact, in the literature on merger policy and practices it is often held as self-evident that merger efficiencies counteract anticompetitive effects and give rise to downward pricing pressure, and does so increasingly. Merger analyses are 
typically based on this presumption, and thus are more concerned with how to measure efficiencies or the threshold of efficiencies to approve a merger (Fisher, et al. 1989 and Salop 1987, 1995). In empirical studies of mergers such as Kim and Singal (1993), it is often assumed that that greater efficiencies lead to lower prices.

The main objective of this chapter is to examine the relationship between the amount of efficiencies, in the form of lower marginal costs, and the price effect of a merger using a Cournot model with differentiated products. In the literature, this relationship has been studied in the context of Bertrand competition with differentiated goods, as well as Cournot competition with a homogeneous good (Farrell and Shapiro 1990, 2010). These studies have supported the conventional belief that larger efficiency gains lead to lower prices. An important finding from our analysis in this chapter is that this negative relationship between efficiency gains and prices does not always hold when firms produce differentiated products and engage in quantity competition. Depending on the degree of substitutability among the products, merger efficiencies can impose either downward or upward pressure on prices. In the former case, merger efficiency reduces prices below the pre-merger level as long as efficiencies are great enough, as the conventional wisdom suggests. In the latter case, however, efficiencies will push prices above the pre-merger level if efficiencies are great enough.

The intuition behind this unconventional result is actually very simple. It stems from the fact that quantities are strategic substitutes. Lower marginal cost brought about by efficiencies encourages output expansion by the merging firms and output contraction by the non-merging firms. This gives rise to ambiguous effects on the prices: While the former puts a downward pressure on prices, the latter creates an upward pricing pressure. 
Under certain conditions related to product substitutability, the latter pricing effect will dominate the former, and the efficiencies, surprisingly, lead to higher prices. This is in sharp contrast to the case of price competition, where a reduction in marginal cost will directly reduce the prices of the merging products, and the non-merging firms will follow suit because of strategic complementarity for prices.

Economists have so far overlooked this price-increasing effect of merger efficiencies. Broadly speaking, this result is related to the small literature on the 'efficiencies offence' (Motta 2004 and Motta and Vasconcelos 2005) that studies anticompetitive effects of efficiencies. In this literature, merger efficiencies force exit (Motta 2004 and Motta and Vasconcelos 2005) or deter entry (Cabral 2003). In addition, Baye and Hunter (2010) informally discuss an example of competition between differentiated products and show that marginal cost savings will not lower prices when prices are determined by localized competition rather than costs.

Nevertheless, the dominant view in both the theoretical and empirical literature on the price effects of mergers is that merger efficiencies are pro-competitive. The empirical study by Hausman, et al. (1994) shows that lower marginal cost will reduce prices as long as post-merger markup does not increase too much. The same idea is conveyed in Farrell and Shapiro (2010) and Werden (1996). Davis and Wilson (2006) show in a merger experiment based on Bertrand competition that cost efficiencies offset upward pricing pressure from increased market power and even reduce prices. The theoretical literature includes Farrell and Shapiro (1990), which study the issue in a Cournot model with a homogeneous good. They show that mergers without cost savings always raise prices, and mergers do have cost savings still raise prices unless the cost savings are 
substantial. Their model is very general; however the assumption of product homogeneity leads to similar results about the effects of efficiencies on prices to those in the price competition models.

Based on the presumption that efficiencies tend to reduce prices, there is also a small literature on pass-through rate that studies how much merger efficiencies would be passed on to consumer prices. Froeb, et al. (2005) and Werden, et al. (2005) are examples of empirical works, while Kate and Niels (2005), Yde and Vita $(1996,2006)$ are theoretical analyses. Since these studies typically assume that firms compete in prices, it then follows that the pass-through rate is always negative, i.e., larger efficiencies lead to lower post-merger prices for merging firms. But this is not true in our model.

In this chapter, we also investigate how the pass-through rate is affected by the intensity of competition. The traditional view is that more intense competition would force firms to pass a bigger share of the efficiency gains to consumers; when the merging firms have stronger market power they tend to pocket more of cost savings. For example, the EC Merger Guidelines (2005, Article 84) state, "The incentive on the part of the merged entity to pass efficiency gains on to consumers is often related to the existence of competitive pressure from the remaining firms in the market". ${ }^{39}$ However, Yde and Vita (1996 and 2006) and Werden, et al. (2005) cast doubt on this view. Yde and Vita (1996, 2006) argue that this view is not supported by economic theories, and they show that the pass-through rate decreases with the intensity of competition: A monopoly will pass through more efficiencies while the merging firms in a competitive industry will simply take the prevailing price as given and pass nothing to consumers. Werden, et al. (2005),

\footnotetext{
${ }^{39}$ For a more detailed discussion about the traditional view on the pass-through rate and market power, see Yde and Vita (1996).
} 
while they largely agree with Yde and Vita (1996), also show that increased competition might have either positive or negative effects on the pass-though rate. Our analysis contributes to this literature by showing that the relation between the pass-through rate and market power depends on the different sources of market power.

Our results are also closely related to the recent literature on the Upward Pricing Pressure (UPP) test. The UPP test is a merger-screening tool to sort out mergers that are likely to lead to higher prices. Using Werden's (1996) idea about the diversion rate, Farrell and Shapiro (2010) formalized the UPP test under Bertrand competition and Moresi (2009) gave the expression of diversion rate based on Cournot competition. Consistent with our foregoing argument, we find that the UPP test tells only part of the story in our model. It cannot reveal the price-increasing aspect of the efficiencies.

The rest of this chapter is organized as follows. In section 3.2 we present our model and derive the main results. In section 3.3 we apply our model to the UPP test; and we carry out a merger simulation to corroborate our unconventional results about efficiencies in section 3.4. Concluding remarks are in section 3.5. The procedures for calculating the equilibrium and corresponding Matlab codes for merger simulation are included in the Appendix.

\subsection{The Model}

The model is the same as the one in Chapter 2 with the only exception that a merger of firms 1 and 2 generates efficiencies. To be more specific, we assume that the merger reduces the marginal costs of the merging firms by a fraction $E \in(0,1)$, so their post- 
merger marginal costs are $(1-E) c .^{40}$ Here we assume savings in marginal cost savings rather than in fixed costs because of the well-known fact that the latter could enhance firm's profitability without changing its pricing or output behavior. To study the impact of efficiencies on prices and outputs, the gains have to manifest themselves through savings in marginal costs.

As in Chapter 2, the demand system is

$$
\begin{aligned}
& p_{1}=\alpha-q_{1}-\gamma q_{2}-\delta \sum_{j=3}^{N} q_{j} \\
& p_{2}=\alpha-\gamma q_{1}-q_{2}-\delta \sum_{j=3}^{N} q_{j} \\
& p_{i}=\alpha-q_{i}-\varphi \sum_{j \neq i} q_{j}(i=3,4, \ldots, N)
\end{aligned}
$$

where $0<\max \{\gamma, \delta, \varphi\}<1$. Furthermore, we shall assume that

$$
A>B, \alpha>c \text { and } E<\bar{E} \equiv \min \left\{\frac{(\alpha-c)(1+\gamma-\varphi)}{\varphi c}, 1\right\}
$$

throughout this chapter where $A=(N-3) \varphi+2$ and $B=(N-2) \delta$. As will become clear later, these assumptions ensure that equilibrium quantities of all firms are positive.

\subsubsection{Pre-Merger Equilibrium}

Firms maximize profits through non-cooperative choices of output levels. Firm 1's optimization problem is

$$
\max _{q_{1}}\left(p_{1}-c\right) q_{1}=\max _{q_{1}}\left(\alpha-q_{1}-\gamma q_{2}-\delta \sum_{j=3}^{N} q_{j}-c\right) q_{1} .
$$

Similarly, Firm 2's optimization problem is

$$
\max _{q_{2}}\left(p_{2}-c\right) q_{2}=\max _{q_{2}}\left(\alpha-\gamma q_{1}-q_{2}-\delta \sum_{j=3}^{N} q_{j}-c\right) q_{2} .
$$

The optimization problem of a non-merging firm $i(=3,4, \ldots N)$ is

\footnotetext{
${ }^{40}$ By this we assume away efficiency spillovers, i.e., the merger does not generate efficiencies for nonmerging firms. Accordingly the cost reductions required for profitability or to regain pre-merger price levels would probably be smaller in our model than in those with spillovers (see e.g. Bian and McFetridge 2000).
} 


$$
\max _{q_{i}}\left(p_{i}-c\right) q_{i}=\max _{q_{i}}\left(\alpha-q_{i}-\varphi \sum_{j \neq i} q_{j}-c\right) q_{i} .
$$

Solving optimization problem (3.5), (3.6) and (3.7), we obtain the pre-merger equilibrium prices and quantities:

$$
\begin{array}{ll}
q_{1}^{*}=q_{2}^{*}=\frac{(A-B)(\alpha-c)}{(2+\gamma) A-2 \varphi B} ; & q_{i}^{*}=\frac{(2+\gamma-2 \varphi)(\alpha-c)}{(2+\gamma) A-2 \varphi B} \\
p_{1}^{*}=p_{2}^{*}=\frac{(\alpha-c)(A-B)}{(2+\gamma) A-2 \varphi B}+c=q_{1}^{*}+c ; & p_{i}^{*}=\frac{(\alpha-c)(2+\gamma-2 \varphi)}{(2+\gamma) A-2 \varphi B}=q_{i}^{*}+c \\
\pi_{1}^{*}=\pi_{2}^{*}=\frac{(\alpha-c)^{2}(A-B)^{2}}{[(2+\gamma) A-2 \varphi B]^{2}}=\left(q_{1}^{*}\right)^{2} ; & \pi_{i}^{*}=\frac{(\alpha-c)^{2}(2+\gamma-2 \varphi)^{2}}{[(2+\gamma) A-2 \varphi B]^{2}}=\left(q_{i}^{*}\right)^{2} .
\end{array}
$$

\subsubsection{Post-merger Equilibrium}

After firms 1 and 2 merge, they coordinate their output decisions by solving

$$
\begin{gathered}
\max _{q_{1}, q_{2}}\left[p_{1}-(1-E) c\right] q_{1}+\left[p_{2}-(1-E) c\right] q_{2}, \\
=\max _{q_{1}, q_{2}}\left[\alpha-q_{1}-\gamma q_{2}-\delta \sum_{j=3}^{N} q_{j}-(1-E) c\right] q_{1} \\
+\left[\alpha-\gamma q_{1}-q_{2}-\delta \sum_{j=3}^{N} q_{j}-(1-E) c\right] q_{2}
\end{gathered}
$$

Non-merging firms' behavior is not changed. The post-merger equilibrium prices and quantities can be shown to be:

$$
\begin{array}{ll}
q_{1}^{e}=q_{2}^{e}=\frac{(\alpha-c)(A-B)+E c A}{2[(1+\gamma) A-\varphi B]} & q_{i}^{e}=\frac{(\alpha-c)(1+\gamma-\varphi)-\varphi E c}{(1+\gamma) A-\varphi B} \\
p_{1}^{e}=p_{2}^{e}=\frac{(\alpha-c)(1+\gamma)(A-B)-E c[(1+\gamma) A-2 \varphi B]}{2[(1+\gamma) A-\varphi B]}+c & p_{i}^{e}=\frac{(\alpha-c)(1+\gamma-\varphi)-\varphi E c}{(1+\gamma) A-\varphi B}+c \\
\pi_{1}^{e}=\pi_{2}^{e}=\frac{(1+\gamma)[(\alpha-c)(A-B)+E c A]^{2}}{4[(1+\gamma) A-\varphi B]^{2}} & \pi_{i}^{e}=\frac{[(\alpha-c)(1+\gamma-\varphi)-\varphi E c]^{2}}{[(1+\gamma) A-\varphi B]^{2}}
\end{array}
$$

The presence of $E$ means that the previous assumptions in Chapter $2(A>B$ and $\alpha>c$ ) are no longer sufficient to guarantee positive quantities of non-merging firms.

Rather we have to either replace the assumption $\alpha>c$ by $\frac{\alpha}{c}>\frac{1+\gamma-(1-E) \varphi}{1+\gamma-\varphi}$, or restrict the 
range of $E$ to make it less than $\frac{(\alpha-c)(1+\gamma-\varphi)}{\varphi c}$ without changing the condition $\alpha>c .{ }^{41} \mathrm{We}$ shall adopt the latter alternative. Note the relative magnitudes of $\frac{(\alpha-c)(1+\gamma-\varphi)}{\varphi c}$ and 1 is indeterminate. This is why we assume $A>B, \alpha>c$ and $E<\bar{E} \equiv \min \left\{\frac{(\alpha-c)(1+\gamma-\varphi)}{\varphi c}, 1\right\}$ throughout this chapter. ${ }^{42}$

It's immediate that both post-merger profits and outputs of the merging firms increase in marginal cost efficiency $E .^{43}$ Due to the strategic substitutability of quantity, the cost efficiency discourages non-merging firms from output expansion, and that is why we have to impose an upper bound $\bar{E}$ to keep non-merging firms from shutting down or exiting.

Note from (3.13) that the prices of the merging firms, $p_{1}^{e}$ may rise or fall with efficiency $E$, depending on the sign of $(1+\gamma) A-2 \varphi B .^{44}$ This suggests the efficiency may tend either to reduce or to increase prices. In other words, merger efficiencies can put either downward or upward pressure on prices.

\footnotetext{
${ }^{41}$ This is derived from (3.12) to ensure that $q_{i}^{e}$ is positive. Intuitively a very efficient merged entity can force less-efficient firms to exit the market. In our settings this means all non-merging firms will exit and the $N$-firm market would be reduced to monopoly after the merger. We thus restrict the range of $E$ to focus on the more interesting case where firms do not exit after the merger.

${ }^{42}$ Note that there is no fixed cost in this model. If however fixed costs are present, the value of $\bar{E}$ will have to be adjusted. Specifically, the presence of fixed costs will make $\bar{E}$ smaller if $\frac{(\alpha-c)(1+\gamma-\varphi)}{\varphi c}<1$, but may or may not reduce $\bar{E}$ if $\frac{(\alpha-c)(1+\gamma-\varphi)}{\varphi c}>1$. This follows the fact that fixed costs do not change firms' behaviour and would thus not change the equilibrium prices or quantities, but they reduce each firm's equilibrium profits by the amount of fixed costs. Therefore, a smaller $E$ is needed in order that non-merging firms earn more (through output expansion) to pay fixed costs (recall that a greater $E$ will force outsiders to reduce outputs by a larger amount). For insiders however, they need greater $E$ to keep balance, so the overall effect on $\bar{E}$ is indeterminate.

${ }^{43}$ This is a typical observation. Farrell and Shapiro (1990) show in a more general model (where firms produce a homogeneous product) that Cournot firms with lower marginal costs produce more.

${ }^{44}$ It is interesting to observe that in our model in Chapter 2 where merger efficiencies are absent, the expression $(1+\gamma) A-2 \varphi B$ determines the sign of the insiders' price change.
} 


\subsubsection{Condition for Profitable Merger}

Before we analyze the effects of the merger, we need to examine the condition under which the merger is profitable. If a merger is not profitable, the firms would not want to merge in the first place, in which case an examination of the effects of the merger becomes an irrelevant exercise. Accordingly, we will study the effects of the merger only under the condition that it is profitable and hence will actually occur in equilibrium.

Lemma 3.1 Define $E_{\pi} \equiv \frac{(\alpha-c)(A-B)}{c A}\left[\frac{(2+2 \gamma) A-2 \varphi B}{(2+\gamma) A-2 \varphi B} \sqrt{\frac{1}{1+\gamma}}-1\right]$; a merger is profitable if $E>E_{\pi}$.

-Proof: The increase in profits for the merging firms is

$$
\begin{aligned}
& \Delta \pi_{1}=\pi_{1}^{e}-\pi_{1}^{*}=\frac{(1+\gamma)[(\alpha-c)(A-B)+E c A]^{2}}{4[(1+\gamma) A-\varphi B]^{2}}-\frac{(\alpha-c)^{2}(A-B)^{2}}{[(2+\gamma) A-2 \varphi B]^{2}} \\
& =(\alpha-c)^{2}(A-B)^{2} \gamma \frac{(1+\gamma) \gamma A^{2}+4 \varphi^{2} B^{2}-4 \varphi(1+\gamma) A B}{4[(1+\gamma) A-\varphi B]^{2}[(2+\gamma) A-2 \varphi B]^{2}} \\
& +\frac{(1+\gamma)\left[(E c A)^{2}+2 E c A(\alpha-c)(A-B)\right][(2+\gamma) A-2 \varphi B]^{2}}{4[(1+\gamma) A-\varphi B]^{2}[(2+\gamma) A-2 \varphi B]^{2}} .
\end{aligned}
$$

Rewriting (3.15) using the common denominator, we find the corresponding numerator is

$$
\begin{aligned}
& (\alpha-c)^{2}(A-B)^{2} \gamma\left[(1+\gamma) \gamma A^{2}-4 \varphi(1+\gamma) A B+4 \varphi^{2} B^{2}\right] \\
& +E c A[E c A+2(\alpha-c)(A-B)](1+\gamma)[(2+\gamma) A-2 \varphi B]^{2} .
\end{aligned}
$$

(3.16) has to be positive for the merger to be profitable. Rewriting (3.16) to obtain

$$
(E c A)^{2} L+E c A(\alpha-c)(A-B) 2 L+K>0
$$

where $L=(1+\gamma)[(2+\gamma) A-2 \varphi B]^{2}$ and

$$
K=(\alpha-c)^{2}(A-B)^{2} \gamma\left[(1+\gamma) \gamma A^{2}-4 \varphi(1+\gamma) A B+4 \varphi^{2} B^{2}\right] .
$$

Consider the equation corresponding to (3.17):

$$
(E c A)^{2} L+E c A(\alpha-c)(A-B) 2 L+K=0 .
$$


Solving (3.18) for $E$, we obtain $E=\frac{(\alpha-c)(A-B)}{c A}\left[-1 \pm \frac{(2+2 \gamma) A-2 \varphi B}{(2+\gamma) A-2 \varphi B} \sqrt{\frac{1}{1+\gamma}}\right]$. We dismiss the negative root and define the other root as

$$
E_{\pi}=\frac{(\alpha-c)(A-B)}{c A}\left[\frac{(2+2 \gamma) A-2 \varphi B}{(2+\gamma) A-2 \varphi B} \sqrt{\frac{1}{1+\gamma}}-1\right]
$$

Thus, (3.16) is positive when $E>E_{\pi}$.

It is immediate from (3.15) that $\pi_{1}^{e}$ and hence $\Delta \pi_{1}$ increases with $E$. Note $E_{\pi} \gtreqless 1$, but $E_{\pi}<1$ must be true for a profitable merger. As shown by (3.19) and will be elaborated on in section 3.2.7, the sign of $E_{\pi}$ can be negative. Lemma 3.1 implies that if $E_{\pi}<0$, any level of efficiencies will make the merger profitable. ${ }^{45}$

\subsubsection{Conditions for Price Increase}

The change in the prices of the merging firms is

$$
\begin{aligned}
& \Delta p_{1}=p_{1}^{e}-p_{1}^{*} \\
& =\frac{(\alpha+c)(1+\gamma) A-(\alpha-c)(1+\gamma) B-2 c \varphi B-E c[(1+\gamma) A-2 \varphi B]}{2[(1+\gamma) A-\varphi B]}-\frac{(\alpha+c+c \gamma) A-(\alpha-c+2 \varphi c) B}{(2+\gamma) A-2 \varphi B} \\
& =\frac{[(1+\gamma) A-2 \varphi B]\{\gamma(\alpha-c)(A-B)-E c[(2+\gamma) A-2 \varphi B]\}}{2[(1+\gamma) A-\varphi B][(2+\gamma) A-2 \varphi B]} .
\end{aligned}
$$

Note that the sign of $\Delta p_{1}$ depends on two parts:

$$
(1+\gamma) A-2 \varphi B
$$

and

$$
\gamma(\alpha-c)(A-B)-E c[(2+\gamma) A-2 \varphi B]
$$

\footnotetext{
${ }^{45}$ If however fixed costs $F$ exist, $E_{\pi}$ will decrease. In other words, a merger will be more likely to be profitable. The reason is that the merger eliminates duplication in fixed costs. To be more specific, the presence of fixed costs would reduce the pre-merger profit for each firm by $F$ while it would decrease postmerger profit for a merging firm by $\frac{F}{2}$. Therefore $\Delta \pi_{1}$ in (3.15) will increase by $\frac{F}{2}$, which is tantamount to a greater $K$ in (3.18). With a greater constant term in a quadratic function, the positive root $\left(E_{\pi}\right)$ will decrease (the other root still remains negative, although a greater $K$ might lead to complex roots).
} 
Note that the expression in (3.21) is identical to the price condition (2.35) in Chapter 2 where merger efficiencies are absent (i.e., $E=0$ ). In the latter case, a merger is profitable only if this expression is positive, as we have shown in Chapter 2. However, with a positive $E$ the range of parameters for a profitable merger is broadened. As a result, it is possible to have a negative (3.21) even with the restriction that the merger has to be profitable.

It is clear that the expression in (3.22) could be negative for a sufficiently large $E$. Define

$$
E_{p}=\frac{\gamma(\alpha-c)(A-B)}{c[(2+\gamma) A-2 \varphi B]} .
$$

Then (3.22) will be positive if $E<E_{p}$. We can rank the magnitude of $E_{p}$ relative to the other two thresholds of $E: E_{\pi}$ in (3.19) and $\bar{E}$ in (3.4).

Lemma 3.2 $E_{\pi}<E_{p}<\frac{(\alpha-c)(1+\gamma-\varphi)}{\varphi c}($ when $\gamma \neq 0)$.

-Proof: That $\quad E_{\pi}=\frac{(\alpha-c)(A-B)}{c A}\left[\frac{(2+2 \gamma) A-2 \varphi B}{(2+\gamma) A-2 \varphi B} \sqrt{\frac{1}{1+\gamma}}-1\right]<E_{p}=\frac{\gamma(\alpha-c)(A-B)}{c[(2+\gamma) A-2 \varphi B]} \quad$ is immediate after some transformation. The proof of $E_{p}<\frac{(\alpha-c)(1+\gamma-\varphi)}{\varphi c}$ is less straightforward. To show this, we need to prove $\frac{\gamma(\alpha-c)(A-B)}{c[(2+\gamma) A-2 \varphi B]}<\frac{(\alpha-c)(1+\gamma-\varphi)}{\varphi c}$, which is equivalent to $\varphi \gamma(A-B)<(1+\gamma-\varphi)[(2+\gamma) A-2 \varphi B]$. Since $\varphi \gamma(A-B)<$ $(1+\gamma-\varphi)(2 \varphi A-2 \varphi B)<(1+\gamma-\varphi)[(2+\gamma) A-2 \varphi B]$, it follows that $E_{p}<\frac{(\alpha-c)(1+\gamma-\varphi)}{\varphi c}$

Note that both $E_{p}$ and $\bar{E}$ are always positive, but $E_{\pi}$ by itself could be either positive or negative. Furthermore, all of $E_{\pi}, E_{p}$ and $\frac{(\alpha-c)(1+\gamma-\varphi)}{\varphi c}$ could be $\gtreqless 1$. While we already 
define $\bar{E} \equiv \min \left\{\frac{(\alpha-c)(1+\gamma-\varphi)}{\varphi c}, 1\right\}$ and know $E_{\pi}<1$ for the merger to be profitable, we do not impose the restriction that $E_{p}<1$. These thresholds of $E$ will be used below in the analysis in sections 3.2.4 and 3.2.5.

Conducting comparative statics on (3.20), we find out how the price change is affected by the efficiencies.

Proposition 3.1 The change in the prices of the merging firms decreases in the size of efficiencies if $\gamma>\frac{2 \varphi B-A}{A}$, and it increases in the size of efficiencies if $\gamma<\frac{2 \varphi B-A}{A}$.

Droof: $\frac{\partial \Delta p_{1}}{\partial E}=\frac{-c[(1+\gamma) A-2 \varphi B]}{2[(1+\gamma) A-\varphi B]}$. It is negative if $\gamma>\frac{2 \varphi B-A}{A}$ and positive if $\gamma<\frac{2 \varphi B-A}{A}$.

\subsubsection{Pass-Through Rate and Compensating Marginal-Cost Reductions}

In our model, the pass-through rate can be represented by $\frac{\partial p_{1}^{e}}{\partial E}$; it measures how much merger efficiencies are passed on to consumers. A glance at $p_{1}^{e}$ in (3.13) tells us that

$$
\frac{\partial p_{1}^{e}}{\partial E}=\frac{-c[(1+\gamma) A-2 \varphi B]}{2[(1+\gamma) A-\varphi B]}
$$

Given our assumptions on the parameters, the denominator of (3.24) is positive. The sign of (3.24) then depends on the sign of its numerator, which, in turn, depends on the sign of (3.21). Since (3.21) can be either positive or negative, the merger efficiencies can impose either downward or upward pressure on prices.

The above observation is in sharp contrast to the conventional view that larger efficiencies lead to lower prices. While this view is correct in models of price competition and in models of quantity competition with a homogeneous product (e.g., Kate and Niels 2005), it does not hold in our model of quantity competition with 
differentiated products. Depending on the sign of $(1+\gamma) A-2 \varphi B$, the pass-through rate could be either positive or negative.

Closely related to the pass-through rate is the concept of Compensating Marginalcost Reductions or CMR (Werden 1996, Werden, et al. 2005), defined as the amount of efficiencies that would restore the pre-merger prices. In our model, CMR is the level of $E$ that makes (3.22) equal 0:

$$
\mathrm{CMR}=\frac{\gamma(\alpha-c)(A-B)}{c[(2+\gamma) A-2 \varphi B]} .
$$

Werden (1996, p.411) pointed out that "[1]arge - typically implausible - cost reductions are necessary to restore premerger prices if the product is highly differentiated and the merging firms compete intensely". In our model, it is easy to show that $\frac{\partial(\mathrm{CMR})}{\partial \gamma}=$ $\frac{(\alpha-c)(A-B)(2 A-2 \varphi B)}{c[(2+\gamma) A-2 \varphi B]^{2}}>0$, meaning that closer merging products require greater efficiencies to restore the pre-merger prices. Also note that CMR is different from pass-through rate in that it is always positive.

\subsubsection{How Efficiency Affects Prices: The Special Case of $\gamma=0$}

In section 3.2.4, we examined the conditions for price increases without directly imposing the restriction that the merger be profitable. In the present section, we will study the effects of efficiencies on post-merger prices taking into account that in equilibrium the merger has to be profitable. We will first look into an extreme case where the two merging products are independent, so that each product does not enter the demand function of the other product, i.e., $\gamma=0$. In this case, there is no competition between the products of firms 1 and 2. If there were no efficiencies (i.e., if $E=0$ ), the merger would not have any effects. However if $E \neq 0$, we have the following results: 
Proposition 3.2 When $\gamma=0$ and $E>0$, mergers are always profitable. Merging firms expand output and non-merging firms reduce output. Moreover, merging firms' prices decrease in $E$ if $\delta<\frac{(N-3) \varphi+2}{2(N-2) \varphi}$, and increase in $E$ if $\delta>\frac{(N-3) \varphi+2}{2(N-2) \varphi}$.

-Proof: Setting $\gamma=0$ in (3.8), (3.12), (3.15) and (3.20), we find:

$$
\Delta \pi_{1}=\frac{(E c A)^{2}+2(\alpha-c)(A-B) E c A}{4(A-\varphi B)^{2}} ; \Delta p_{1}=\frac{-E c(A-2 \varphi B)}{2(A-\varphi B)} ; \Delta q_{1}=\frac{E c A}{2(A-\varphi B)} .
$$

Therefore a positive $E$ expands the merging firms' outputs and makes the merger profitable. However, the unusual price effects may still exist: if $A-2 \varphi B<0$, then greater $E$ raises the prices of merging firms. ${ }^{46}$ The inequality $A-2 \varphi B<0$ holds when $\delta>\frac{(N-3) \varphi+2}{2(N-2) \varphi}$. In other words, if the latter condition is satisfied, a larger reduction in the marginal costs of the merging firms only results in their higher prices.

The intuition behind the price effect of merger efficiencies can be understood with the help of the merging firms' demand functions: $p_{1}=\alpha-q_{1}-\delta \sum_{j=3}^{N} q_{j}=\alpha-q_{1}-$ $B q_{i}$. Note $\frac{\partial q_{1}^{e}}{\partial E}>0$ and $\frac{\partial q_{i}^{e}}{\partial E}<0$, and $\left|\frac{\partial\left(B q_{i}\right)}{\partial E}\right|>\left|\frac{\partial q_{1}}{\partial E}\right|$ if $A-2 \varphi B<0$ or equivalently $\delta>\frac{(N-3) \varphi+2}{2(N-2) \varphi}$. Accordingly, a greater $E$ raises the equilibrium price $p_{1}^{e}$ when $\delta>$ $\frac{(N-3) \varphi+2}{2(N-2) \varphi}$. In other words, a larger reduction in marginal cost induces the merging firms to produce more, which, in turn, causes each non-merging firm to produce less due to strategic substitutability of quantity. The former pushes the merging firms' prices down, while the latter pushes the prices up. The latter effect is large when the substitutability

\footnotetext{
${ }^{46}$ When the number of firms in the industry is 3 , this never happens: $A-2 \varphi B=2-2 \varphi \delta>0$ and the greater efficiency the lower price. But if, say, $N=4$, then $A-2 \varphi B=2+\varphi-4 \varphi \delta<0$ if $\min \{\varphi, \delta\}<$ $1<\frac{1}{4}\left(\sqrt{\varphi^{2}+32 \varphi \delta}-\varphi\right)$. Note $\min \{\varphi, \delta\}<\frac{1}{4}\left(\sqrt{\varphi^{2}+32 \varphi \delta}-\varphi\right)$ requires that $4 \delta>3 \varphi>2 \delta$. More generally $A-2 \varphi B=(N-3) \varphi+2 \beta^{2}-2(N-2) \varphi \delta$, the derivative of which w.r.t $N$ is $(1-2 \delta) \varphi$. So if $1>2 \delta$ then $A-2 \varphi B$ is always positive; otherwise, $A-2 \varphi B$ could be negative when $N$ is large.
} 
between the merging firms' products and the non-merging firms' products is high, i.e., when $\delta$ is large. Consequently, this effect dominates when $\delta$ is sufficiently large in the sense that $\delta>\frac{(N-3) \varphi+2}{2(N-2) \varphi}$.

In addition, note that $E_{\pi}=E_{p}=0$ when $\gamma=0$. This also confirms that a merger between independent products is always profitable for any $E>0$.

\subsubsection{How Efficiency Affects Prices: General Case}

We now proceed to examine the general case where $\gamma$ is positive. Recall that the direction of change in the prices of the merging firms depends on the signs of (3.21) and (3.22). The sign of (3.21), in turn, can be either positive or negative, depending on the size of $\gamma$. Accordingly, we will study each of these two cases separately.

Case 1. $(1+\gamma) A-2 \varphi B>0$

$E>E_{\pi}$ is needed to ensure that the merger is profitable. Using Lemmas 3.1 and 3.2, we can illustrate the relationships between the profit change, the price change and the efficiencies as in Figure 3.1.
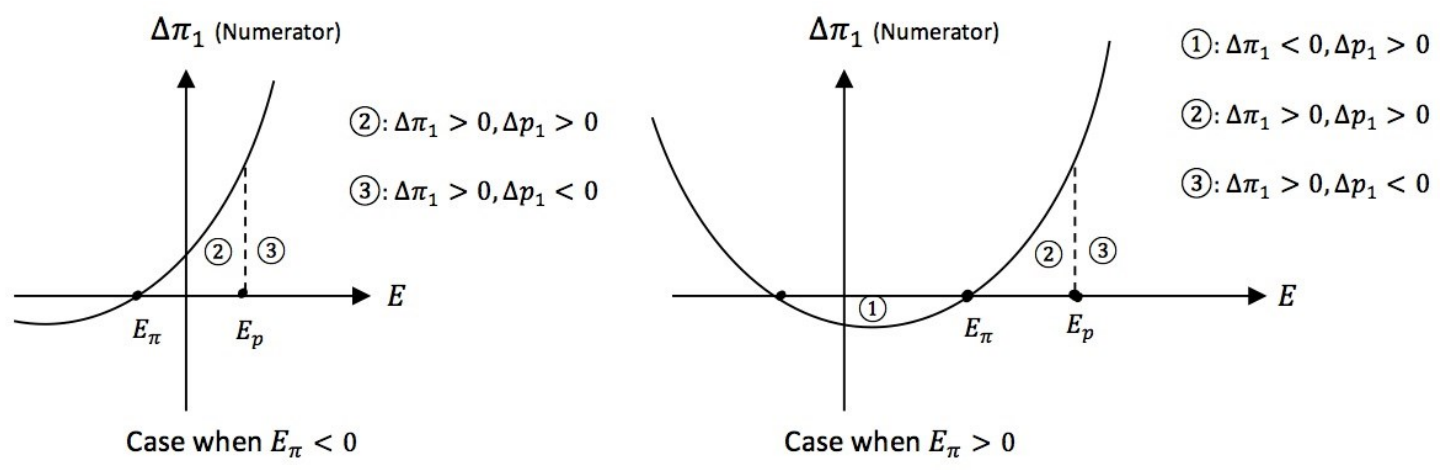

Note: (3) will disappear when $E_{p}>1$.

Figure 3.1 Price and Profit Change When $(1+\gamma) \mathrm{A}-2 \varphi \mathrm{B}>0$ 
Figure 3.1 contains two graphs, for the cases where $E_{\pi}$ is negative and positive. This is necessary because both cases are possible when $(1+\gamma) A-2 \varphi B>0$. To see this, note (3.19) shows that $E_{\pi}<0$ if and only if $\frac{(2+2 \gamma) A-2 \varphi B}{(2+\gamma) A-2 \varphi B} \sqrt{\frac{1}{1+\gamma}}<1$. The latter condition is equivalent to the merger profitability condition (2.29) in Chapter 2. Recall the merger profitability is undetermined under the condition $(1+\gamma) A-2 \varphi B>0$ in Chapter 2 (where efficiencies are assumed away). This implies that both $E_{\pi}<0$ and $E_{\pi}>0$ are possible.

From Figure 3.1 and Lemmas 3.1 and 3.2, we obtain the following results about the effect of the merger on prices and its relationship to the size of efficiencies.

Proposition 3.3A. Suppose $\gamma>\frac{2 \varphi B-A}{A}$. Moreover, suppose $E>E_{\pi}$ so that the merger is profitable.

(a) If $E_{p}<1$, then the merger raises the merging firms' prices, i.e., $p_{1}^{e}>p_{1}^{*}$, for $E \in\left(E_{\pi}, E_{p}\right)$. It reduces their prices, i.e., $p_{1}^{e}<p_{1}^{*}$, for $E \in\left(E_{p}, \bar{E}\right)$.

(b) If $E_{p}>1$, then the merger raises the merging firms' prices, i.e., $p_{1}^{e}>p_{1}^{*}$, for all $E \in\left(E_{\pi}, \bar{E}\right)$

-Proof: The condition $\gamma>\frac{2 \varphi B-A}{A}$ is equivalent to $(1+\gamma) A-2 \varphi B>0$. Recall from earlier discussion that $E_{p}=\frac{\gamma(\alpha-c)(A-B)}{c[(2+\gamma) A-2 \varphi B]} \lesseqgtr 1$. If $E_{p}>1$, (3.22) is positive so that area (3) in Figure 3.1 does not exist for $E<1$. Moreover, $E_{p}<\min \left\{\frac{(\alpha-c)(1+\gamma-\varphi)}{\varphi c}, 1\right\}=\bar{E}$ because $E_{p}<\frac{(\alpha-c)(1+\gamma-\varphi)}{\varphi c}$ as shown in Lemma 3.2. The relation between $E_{\pi}, E_{p}$, and $\frac{(\alpha-c)(1+\gamma-\varphi)}{\varphi c}$ is also shown in Lemma 3.2. The relation between $E$ and $E_{\pi}$ for profit 
change is shown in section 3.2.3, and the relation between $E$ and $E_{p}$ for price change is shown in section 3.2.4.

Proposition 3.3A suggests that when the products of the merging firms are close substitutes (i.e. $\gamma$ is large), a merger that generates large efficiencies can lead to lower prices. For this to occur, however, $E_{p}$ has to be less than 1 . Note that $E_{p}$ itself depends on $\gamma$ and other parameters. To gain a further understanding of the role played by demand substitutability, we rewrite Proposition 3.3A in terms of the primary parameters of the model, as follows.

Proposition 3.3B (a) If $\frac{2 \varphi B-A}{A}<\gamma<\frac{2 c(A-\varphi B)}{(\alpha-c)(A-B)-c A}$, then the merger raises the merging firms' prices, i.e., $p_{1}^{e}>p_{1}^{*}$, for $E \in\left(E_{\pi}, E_{p}\right)$. It reduces their prices, i.e., $p_{1}^{e}<p_{1}^{*}$, for $E \in\left(E_{p}, \bar{E}\right)$.

(b) If $\gamma>\frac{2 c(A-\varphi B)}{(\alpha-c)(A-B)-c A}$, then the merger raises the merging firms' prices, i.e., $p_{1}^{e}>p_{1}^{*}$, for all $E \in\left(E_{\pi}, \bar{E}\right)$.

-Proof: $E_{p}<1$ is equivalent to $\gamma<\frac{2 c(A-\varphi B)}{(\alpha-c)(A-B)-c A}$. The results then follow from Proposition 3.3A.

From Proposition 3.3B, we see that while $\gamma$ needs to be large enough for the possibility of lower prices, it cannot be too large. If $\gamma$ is too large, mergers always raise prices. In other words, efficiencies can lead to lower prices after the merger if the degree of substitutability between the two products is in an intermediate range. If the latter is too large or too small, the merger always leads to higher prices for any amount of efficiencies. 
Recall from (3.24) that the pass-through rate $\frac{\partial p_{1}^{e}}{\partial E}$ is negative when $(1+\gamma) A-$ $2 \varphi B>0$. Therefore, this case delivers a standard outcome about the effects of cost efficiencies: larger efficiencies lead to lower post-merger prices. What we learn from Proposition 3.3 is that this does not mean that post-merger prices will necessarily be lower than pre-merger prices for sufficiently large efficiencies. The latter will occur only if the degree of substitutability of the merging firms' products is within a certain range.

Case 2. $(1+\gamma) A-2 \varphi B<0$

The above condition is equivalent to $\gamma<\frac{2 \varphi B-A}{A}$, which implies that the merging firms produce distant substitutes (or low substitutability between their products). As we have seen in Chapter 2, a merger without efficiencies is not profitable in this case. This implies that $E_{\pi}>0$; a merger is profitable only if it generates some efficiencies.

Recall from (3.24) that in this case $\frac{\partial p_{1}^{e}}{\partial E}>0$; an increase in efficiencies creates an upward pressure on post-merger prices. This creates the interesting possibility that a merger leads to lower prices when efficiencies are small, but higher prices when efficiencies are large, as we can see from the following proposition. 


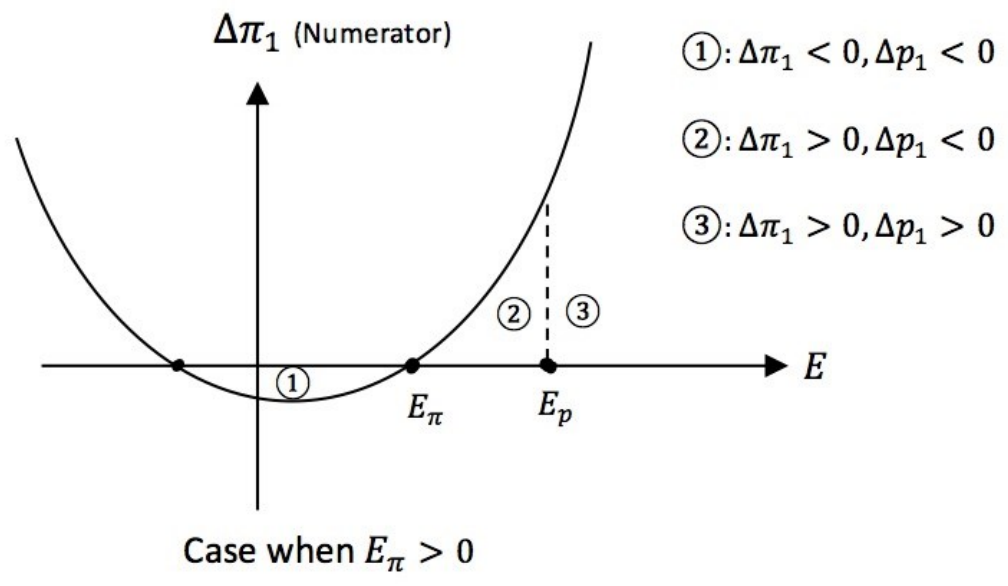

Note: (3) will disappear when $E_{p}>1$.

Figure 3.2 Price and Profit Change When $(1+\gamma) \mathrm{A}-2 \varphi \mathrm{B}<0$

Proposition 3.4A Suppose $\gamma<\frac{2 \varphi B-A}{A}$. Moreover, suppose $E>E_{\pi}$ so that the merger is profitable.

(a) If $E_{p}<1$, then the merger reduces merging firms' prices, i.e., $p_{1}^{e}<p_{1}^{*}$, for $E \in\left(E_{\pi}, E_{p}\right)$. It raises their prices, i.e., $p_{1}^{e}>p_{1}^{*}$, for $E \in\left(E_{p}, \bar{E}\right)$.

(b) If $E_{p}>1$, then the merger reduces merging firms' prices, i.e., $p_{1}^{e}<p_{1}^{*}$, for all $E \in\left(E_{\pi}, \bar{E}\right)$

Proposition 3.4A suggests that the price effects of the merger depend on the value of $E_{p}$. As we did in Proposition 3.3, we can rewrite Proposition 3.4A in terms of the primary parameters of the model.

Proposition 3.4B (a) If $\gamma<\min \left\{\frac{2 \varphi B-A}{A}, \frac{2 c(A-\varphi B)}{(\alpha-c)(A-B)-c A}\right\}$, then the merger reduces the merging firms' prices, i.e., $p_{1}^{e}<p_{1}^{*}$, for $E \in\left(E_{\pi}, E_{p}\right)$. It raises their prices, i.e., $p_{1}^{e}>p_{1}^{*}$, for $E \in\left(E_{p}, \bar{E}\right)$. 
(b) If $\frac{2 c(A-\varphi B)}{(\alpha-c)(A-B)-c A}<\gamma<\frac{2 \varphi B-A}{A}$, then the merger reduces the merging firms' prices, i.e., $p_{1}^{e}<p_{1}^{*}$, for all $E \in\left(E_{\pi}, \bar{E}\right)$.

The proofs of Propositions 3.4A and 3.4B are similar to those of Propositions 3.3A and 3.3B. Hence, they are omitted.

Both versions of Proposition 3.4 present situations with respect to price effects that are opposite to those of Proposition 3. The basic logic follows what we have demonstrated in our special case of zero $\gamma$ : efficiencies create upward quantity pressure for the merging firms and downward quantity pressure for the non-merging firms, and in turn downward pricing pressure and upward pricing pressure respectively on the merging firms. When $(1+\gamma) A-2 \varphi B<0$, the latter outweighs the former and creates overall upward pricing pressure and therefore $\frac{\partial p_{1}^{e}}{\partial E}>0$. As long as $E<E_{p}$ this upward pricing pressure is not so great and cannot push $p_{1}^{e}$ above its premerger level, but if $E>E_{p}$ then the upward pressure is so strong that price will rise beyond the pre-merger level. ${ }^{47}$

This result is in stark contrast to that from models of Bertrand competition, where cost efficiencies directly translate into lower prices for both merging and non-merging firms, due to the strategic complementarity of prices. Thus, efficiencies always create downward pricing pressure when firms compete in prices. ${ }^{48}$

\footnotetext{
${ }^{47}$ Farrell and Shapiro (2010, p.12) acknowledge that the merger firm's cost reduction would increase the partner upward pricing pressure. However, they insist that this 'feedback effect' does not change their central claim that cost efficiecy tends to bring down prices.

${ }^{48}$ Our analysis is also in contrast to Cournot model with a homogeneous product which bears similar results as Bertrand competition. Farrell and Shapiro (1990) show in their inequality (7) that the merging firm's price will rise if $p_{1}^{*}-c>E c$ (in our notation), and the same result is shown in Moresi (2009), inequality (3). By equating $\gamma=\delta=\varphi=1$ for the homogeneous product model and substituting our results of $p_{1}^{*}$, this is equivalent to $\alpha-c>E c(N+1)$; in our own results about $\Delta p_{1}$ in (11) we can show (also by equating $\gamma=\delta=\varphi=1)$ that price will increase if $[(1+\gamma) A-2 \varphi B]\{\gamma(\alpha-c)(A-B)-E c[(2+$ $\gamma) A-2 \varphi B]\}>0 \Leftrightarrow 2[(\alpha-c)-E c(N+1)]>0$. It is clear that our result regarding price effect is identical to Farrell and Shapiro (1990) and Moresi (2009) if only homogeneous product is considered. The
} 


\subsubsection{The Relationship between Market Power and Pass-through Rate}

In the case where a merger is expected to generate efficiencies, an antitrust agency may care about how much of those efficiencies would be passed on to consumers. In this regard, an interesting question is how the pass-through rate is affected by the intensity of competition. The traditional view on this is that more intense competition would force more efficiencies to be passed on, but recently some authors (Werden, et al. 2005, Yde and Vita 1996, 2006) have argued that the pass-through rate increases with market power.

In our model, the parameters $\gamma, \delta$ and $N$ are indicators of market power or competition. To be more specific, $\gamma$ measures the merging firms' market power because it indicates the degree of competition between their products and the merger eliminates that competition. Parameter $\delta$ measures how much competition that the merging firms face from non-merging firms; and $N$ measures market structure. Some simple calculus shows that $\frac{\partial\left(\frac{\partial p_{1}^{e}}{\partial E}\right)}{\partial \gamma}=\frac{-c \varphi A B}{2[(1+\gamma) A-\varphi B]^{2}}<0 \quad, \quad \frac{\partial\left(\frac{\partial p_{1}^{e}}{\partial E}\right)}{\partial \delta}=\frac{c(1+\gamma) \varphi A}{2[(1+\gamma) A-\varphi B]^{2}}>0 \quad$ and $\quad \frac{\partial\left(\frac{\partial p_{1}^{e}}{\partial E}\right)}{\partial N}=$ $\frac{-c(1+\gamma) \varphi(\varphi B-\delta A)}{2[(1+\gamma) A-\varphi B]^{2}} \gtreqless 0$. Even though the first two derivatives are definitely signed, we do not have a simple and unambiguous answer to the question about how market power affects the pass-through rate $\left(\frac{\partial p_{1}^{e}}{\partial E}\right)$ because the latter can be either positive or negative. What we can say, in light of these derivatives, is that the effect of market power on the pass-through rate is different, depending on the source of market power.

\subsubsection{Change of Quantity}

The change in merging firms' output level is 


$$
\Delta q_{1}=q_{1}^{e}-q_{1}^{*}=A \frac{E c[(2+\gamma) A-2 \varphi B]-\gamma(\alpha-c)(A-B)}{2(1+\gamma)[(1+\gamma) A-\varphi B][(2+\gamma) A-2 \varphi B]}
$$

and the change in industry output is

$$
\Delta q=2 q_{1}^{e}+(N-2) q_{i}^{e}-2 q_{1}^{*}-(N-2) q_{i}^{*}=(2-\varphi) \frac{E c[(2+\gamma) A-2 \varphi B]-\gamma(\alpha-c)(A-B)}{[(1+\gamma) A-\varphi B][(2+\gamma) A-2 \varphi B]} .
$$

It is obvious that the merging firm's output changes in the same direction as industry output, and both increase in efficiencies $E$. Also note the common terms in both numerators, $E c[(2+\gamma) A-2 \varphi B]-\gamma(\alpha-c)(A-B)$, is just the negative of (3.22). This implies that, in the case $(1+\gamma) A-2 \varphi B>0$, the merging firm's prices and outputs change in opposite direction as efficiencies grow larger. In the case $(1+\gamma) A-2 \varphi B<$ 0 , the prices and outputs change in same direction.

\subsection{An Application: UPP Test}

The 2010 Guidelines of the United States reflect a drift away from the standard twostep framework for assessing horizontal mergers. The main differences are more lengthy articulations on unilateral effects analysis and that Agencies are seeking more direct upward pricing evidence instead of relying solely on market delineation. These ideas are central to the so-called Upward Pricing Pressure (UPP) test put forward by Farrell and Shapiro (2010) as an easy and efficient alternative to the present procedure. As a prescreening test, the UPP test has its own value in that it is simple and it depends only on pre-merger data, thus avoiding a full evaluation of equilibrium adjustment. Although the 2010 Guidelines do not explicitly include the UPP test, the ideas have been encompassed.

The principle of the UPP test is the weighing of two offsetting effects in post-merger pricing: the anticompetitive effects that tend to push up prices and merger-generated efficiencies that exert downward pressure on pricing. Concerning the upward pricing 
pressure, Farrell and Shapiro (2010) regard a merger between Product 1 and 2 as raising the opportunity cost of selling additional units of Product 1 because this is at the expense of Product 2's loss of sales. While this idea (as well as the claim that efficiencies tend to reduce prices) is intuitively so true and the UPP test fits well with models of Bertrand competition with differentiated products, it could give rise to ambiguities in models of Cournot competition. Farrell and Shapiro (2010 section F, pp. 15-17) remark that "so is (extremely general) the idea that this increase in marginal cost will elevate the price of Product 1 and harm consumers". ${ }^{49}$ As we have shown with respect to the pricing effects of efficiencies, this claim is not true in our model. An increase in the marginal cost of firm 1 will curb its production while stimulating the rivals' production. The former tends to increase product 1's price while the latter tends to decrease it. When $(1+\gamma) A<2 \varphi B$, the latter effect will dominate the former and thus the price of product 1 will fall.

In this section, we demonstrate the relation between the change in the prices of the merging firms and the UPP test in our model. We will show that the UPP term is only a part of the price change, and the UPP test works only if $(1+\gamma) A-2 \varphi B$ is positive, but it leads to the wrong conclusion otherwise.

\subsubsection{The UPP Test}

At the core of the UPP test is the diversion ratio (hereafter DR, Farrell and Shapiro 2010, Shapiro 1996, Werden 1996), denoted by $D_{12}$. It is defined as $\frac{\partial q_{2}}{\partial q_{1}}$ to measure the degree of competition between two merging products: how much of product 1's lost sales due to a post-merger price-rise will go to product 2 .

\footnotetext{
${ }^{49}$ They also suggest (Footnote 33, p.16) that their findings apply to Cournot model (with a homogeneous good) with just an artificial diversion ratio of unity. However, as we will show below, they need more conditions than this with differentiated goods.
} 
The UPP test compares $D_{12}\left(p_{1}^{*}-c\right)$ that creates upward pricing pressure with $E c$ which causes downward pricing pressure. ${ }^{50}$ To evaluate the effectiveness of this test in a market where firms produce differentiated products and compete in quantities, we need to compare the UPP test with our conditions of price increase.

In a model of Bertrand competition, the diversion ratio can be written as $\frac{\partial q_{2}}{\partial p_{1}} / \frac{\partial q_{1}}{\partial p_{1}}$. Moresi (2009) adapts this idea in a model of Cournot competition, and defines the ratio, $\widetilde{D}_{12}=\frac{\partial p_{2}}{\partial q_{1}} / \frac{\partial p_{2}}{\partial q_{2}}$, as the 'price DR'. ${ }^{51}$

If we apply this price DR into the 'cannibalization tax' in our Cournot model, we have the UPP test as the following:

$$
\begin{aligned}
\mathrm{UPP} & =\widetilde{D}_{12}\left(p_{2}^{*}-c\right)-E c=\gamma \frac{(\alpha-c)(A-B)}{(2+\gamma) A-2 \varphi B}-E c . \\
& =\frac{\gamma(\alpha-c)(A-B)-E c[(2+\gamma) A-2 \varphi B]}{(2+\gamma) A-2 \varphi B}
\end{aligned}
$$

Now (3.20) can be rewritten as

$$
\Delta p_{1}=\frac{[(1+\gamma) A-2 \varphi B]\{\gamma(\alpha-c)(A-B)-E c[(2+\gamma) A-2 \varphi B]\}}{2[(1+\gamma) A-\varphi B][(2+\gamma) A-2 \varphi B]}=\frac{[(1+\gamma) A-2 \varphi B]}{2[(1+\gamma) A-\varphi B]} \cdot \mathrm{UPP}
$$

From (3.29), it is clear that the UPP test cannot gauge the price effects in our Cournot model with differentiated products for exactly the same reason as we have discussed in section 3.2.3: the sign of $(1+\gamma) A-2 \varphi B$ can be either positive or negative. Moresi (2009, p.2 and footnote 7) suggests that the UPP test "could be useful for gauging potential unilateral effects in a variety of industries (including Cournot and bidding

\footnotetext{
${ }^{50}$ The UPP that takes into account feedback of merging partner's cost efficiency is $D_{12}\left[p_{1}^{*}-(1-E) c\right]-$ $E c$. This will flag mergers with only moderate cost efficiencies that could have passed the test using $D_{12}\left(p_{1}^{*}-c\right)-E c$. The former is more accurate and stricter a criterion, but does not change the nature that greater efficiency pushes down prices. For our purposes we are concerned only with the latter for simplification. See Farrell and Shapiro (2010).

${ }^{51}$ Diversion ratio in Bertrand competition is also called 'quantity DR'.
} 
competitions)". While this remark holds for Cournot model with homogeneous goods, it is not true in our model of differentiated products.

\subsubsection{The Relationship between $\gamma$ and UPP}

It is fairly easy to show that $\frac{\partial(\mathrm{UPP})}{\partial \gamma}=\frac{(\alpha-c)(A-B)(2 A-2 \varphi B)}{[(2+\gamma) A-2 \varphi B]^{2}}>0$. Thus, the UPP in our model does increase in the degree of substitutability between the merging products, meaning that the closer the merging products, the greater the upward pricing pressure. ${ }^{52}$ However, (3.29) tells us that UPP is not the same as the price increase, and we are unable to determine the sign of $\frac{\partial \Delta p_{1}}{\partial \gamma}$ in our model. ${ }^{53}$ Thus, we are not able to say whether the price change increases in the closeness of the merging products.

\subsection{Simulation}

In this section we carry out a merger simulation to verify our foregoing results. For our purpose, we are concerned only with the unconventional result that greater efficiencies lead to higher prices. As a result, we set $N=10, \gamma=0.4, \delta=0.9, \varphi=0.8$, $\alpha=2, c=1$. We choose two values for efficiencies: $E=0.3$ and $E=0.4$. In both instances, all assumptions in (3.4) are satisfied: $A=7.6>B=7.2, \alpha>c, E<\bar{E} \equiv$ $\min \left\{\frac{(\alpha-c)(1+\gamma-\varphi)}{\varphi c}, 1\right\}=\min \{0.75,1\}$; the profitability condition $E>E_{\pi}=0.012$ is also satisfied; further $(1+\gamma) A-2 \varphi B=-0.88<0$ and $\gamma(\alpha-c)(A-B)-E c[(2+\gamma) A-$

\footnotetext{
${ }^{52}$ It happens that $\mathrm{UPP}=\mathrm{CMR} \cdot c-E c$, so $\frac{\partial(\mathrm{UPP})}{\partial \gamma}=\frac{\partial(\mathrm{CMR})}{\partial \gamma} \cdot c$.

${ }^{53}$ However, when efficiencies are zero, $\frac{\partial \Delta p_{1}^{m}}{\partial \gamma}>0$. See Chapter 2.
} 
$2 \varphi B]=0.16-6.72 E<0$ so that unusual price effects occur. The numerical model is then solved using Matlab. ${ }^{54}$

Table 3.1: Simulation when $\boldsymbol{E}=\mathbf{0 . 3}$

\begin{tabular}{|c|c|c|c|c|c|c|c|c|c|}
\hline Firm & $p_{i}^{*}$ & $q_{i}^{*}$ & $\pi_{i}^{*}$ & $p_{i}^{e}$ & $q_{i}^{e}$ & $\pi_{i}^{e}$ & $\Delta p_{i}$ & $\Delta q_{i}$ & $\Delta \pi_{i}$ \\
\hline$i=1$ & 1.0595 & 0.0595 & 0.0035 & 1.0844 & 0.2746 & 0.0232 & 0.0249 & 0.2151 & 0.0197 \\
\hline$i=2$ & 1.0595 & 0.0595 & 0.0035 & 1.0844 & 0.2746 & 0.0232 & 0.0249 & 0.2151 & 0.0197 \\
\hline$i=3, \ldots, 10$ & 1.119 & 0.119 & 0.0142 & 1.0738 & 0.0738 & 0.0054 & -0.0452 & -0.0452 & -0.0088 \\
\hline
\end{tabular}

Table 3.2: Simulation when $\boldsymbol{E}=\mathbf{0 . 4}$

\begin{tabular}{|c|c|c|c|c|c|c|c|c|c|}
\hline Firm & $p_{i}^{*}$ & $q_{i}^{*}$ & $\pi_{i}^{*}$ & $p_{i}^{e}$ & $q_{i}^{e}$ & $\pi_{i}^{e}$ & $\Delta p_{i}$ & $\Delta q_{i}$ & $\Delta \pi_{i}$ \\
\hline$i=1$ & 1.0595 & 0.0595 & 0.0035 & 1.0934 & 0.3525 & 0.0329 & 0.0339 & 0.293 & 0.0294 \\
\hline$i=2$ & 1.0595 & 0.0595 & 0.0035 & 1.0934 & 0.3525 & 0.0329 & 0.0339 & 0.293 & 0.0294 \\
\hline$i=3, \ldots, 10$ & 1.119 & 0.119 & 0.0142 & 1.0574 & 0.0574 & 0.0033 & -0.0616 & -0.0616 & -0.0109 \\
\hline
\end{tabular}

The results from the simulation are presented in Tables 3.1 and 3.2. In both tables, we see that the merger is profitable and it raises the prices of the merging firms. If we compare across the two tables, we see that as the efficiencies increase from 0.3 to 0.4 , the price change grows larger from 0.025 to 0.034 . Incidentally, note that the merger reduces the profits and prices of the non-merging firms.

\subsection{Conclusions}

We use a Cournot model with differentiated products to study the effects of mergers in the presence of merger efficiencies. We find that marginal cost efficiency broadens the range of degree of substitutability between merging products for merger to be profitable. We confirm the conventional wisdom that merger efficiencies stimulate greater outputs and impose downward pressure on prices when the merging products are sufficiently close rivals. More importantly we also find that, when the merging products are more

\footnotetext{
${ }^{54}$ See Appendix D for the technical details of the merger simulation, including the Matlab codes used to compute the pre-merger and post-merger equilibriums.
} 
distant rivals, an unconventional price effect arises. In this case merger efficiencies generate upward pricing pressure and can push prices beyond the pre-merger level if efficiencies are great enough.

This unconventional price effect of efficiencies has a simple intuitive explanation. Unlike Bertrand competition, efficiencies in Cournot competition with differentiated products expand the merging firms' outputs and reduce non-merging firms' outputs. While the former creates downward pricing pressure, the latter imposes upward pricing pressure on the merging products. Under certain conditions the latter dominates the former, leading to higher prices for the merging products.

By applying our findings to the UPP test and pass-through rate, we find that these concepts do not work well in our model. Although they fit nicely with Bertrand competition, the results could be reversed in markets where firms produce differentiated products and compete in quantities.

Some caveats about this study have to be made. In our model greater efficiencies will push up prices only when the merging products are sufficiently 'dissimilar' in characteristics and the efficiencies are sufficiently large. In other words, the unusual results are more likely to occur when the merger between more 'distant' products creates greater marginal cost efficiencies. One might dismiss the significance of this result by arguing that efficiencies are more likely to arise when the merging products share more common attributes. In response, we would argue that our results are relevant to situations where products with dissimilar consumer attributes share similar or complementary technologies or distribution channels. 
In spite of the possible unconventional effect of efficiency on merging firms' prices, we cannot simply conclude that efficiency would thus lessen competition. In that case, the non-merging firms' prices would drop more than the price increases by the merging firms. 


\section{Appendices}

\section{Appendix A Proof of Proposition 1.2}

This proof is carried out by comparing $F_{M}$ and $F_{C}$ with pre-merger strategy $F$. Note that to compare the pre-merger and post-merger strategies, we are only concerned with the situation where there is overlap between the pair $\left(F_{M}\right.$ or $\left.F_{C}, F\right)$. Since firm $\mathrm{C}$ has a smaller capacity than firm M, $F_{C}>F_{M}$ (Kreps and Scheinkman 1983).

- First, consider the cases $p^{L}<p<P(2 K)<p^{H}$ and $p^{H}<P(2 K)$.

There are several possibilities depending on the value of $p^{L}$. If $p^{L}>p^{h}$, then there is no overlap between $\left(p^{l}, p^{h}\right)$ and $\left(p^{L}, p^{H}\right)$; or, if $p^{L}<p^{h}<p$, there is overlap between $\left(p^{l}, p^{h}\right)$ and $\left(p^{L}, p^{H}\right)$, but as $p^{h}<p$, the relevant price $p$ does not fall in the overlap. In both situations pre-merger strategy $F$ is stochastically dominated by those after the merger (in the relevant price range).

The remaining two possibilities are $p^{l}<p^{L}<p<p^{h}<p^{H}<P(2 K)$ and $p^{l}<$ $p^{L}<p<p^{h}<P(2 K)<p^{H}$. The only difference between these two situations is whether $p^{H}$ is greater or less than $P(2 K)$. However, the position of $p^{H}$ relative to $P(2 K)$ does not matter to our analysis here.

Now consider $F_{M}=\frac{\left(p-p^{L}\right) K}{p(3 K-D)}, F_{C}=\frac{2\left(p-p^{L}\right) K}{p(3 K-D)}, F=\sqrt{\frac{\left(p-p^{l}\right) K}{p(3 K-D)}}$. It is obvious that $F_{M}<F^{2}<F$ for any $p \in\left(p^{l}, p^{H}\right)$, which means firm M behaves less aggressively than firm $\mathrm{A}, \mathrm{B}$, or $\mathrm{C}$ does before the merger (firm $\mathrm{M}$ tends to undercut price with a smaller probability than before the merger). In the meantime, $F_{C}=2 F_{M}<2 F^{2} \lessgtr F$; more 
specifically, if $F \leq \frac{1}{2}$, then $2 F<1, F_{C}=2 F_{M}<2 F^{2}=2 F \cdot F<F, F_{C}$ is less than $F$ at any price level.
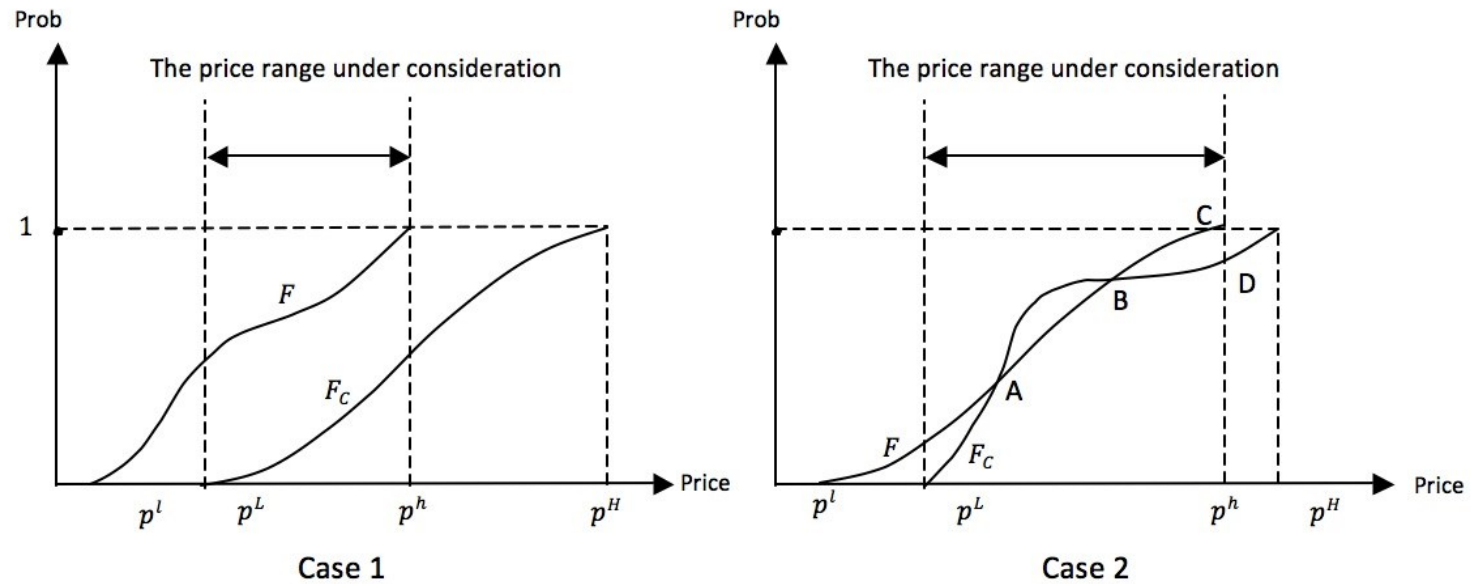

Figure A1

For $p$ such that $F(p)>\frac{1}{2}$, the comparison between $F_{c}$ and $F$ is not immediate. As illustrated in Figure A1, there are two possibilities. The first case is that $F_{C}$ dominates $F$ at all overlapping price levels, and the second, if $F$ dominates $F_{C}$ in some range, then $F$ and $F_{C}$ would intersect at least twice. It's not possible that they only cross once, because we know that $\forall F \leq \frac{1}{2}, F>F_{C}$, and point $\mathrm{C}$ must be higher than point $\mathrm{D}$ (if point $\mathrm{D}$ is as high as point $\mathrm{C}$, i.e., $F_{C}\left(p^{h}\right)=F\left(p^{h}\right)=1$, then $\forall p \in\left(p^{h}, p^{H}\right), F_{C}(p)$ is measure- 0 , which is not possible).

We solve for the slope of $F$ and $F_{C}$ for all $p$ and obtain:

$\frac{d F_{C}}{d p}=\frac{2 K p^{L}\left(3 K-D-P D^{\prime}\right)+2 p^{2} K D^{\prime}}{p^{2}(3 K-D)^{2}}$ and $\frac{d F}{d p}=\frac{K p^{l}\left(3 K-D-P D^{\prime}\right)+p^{2} K D^{\prime}}{2 \sqrt{\frac{(p-p l) K}{p(3 K-D)}} p^{2}(3 K-D)^{2}}$.

If the slope of $F_{C}$ is everywhere (in the overlap) greater than that of $F$, then we must have 
$\frac{d F_{C}}{d p}=\frac{2 K p^{L}\left(3 K-D-P D^{\prime}\right)+2 p^{2} K D^{\prime}}{p^{2}(3 K-D)^{2}}>\frac{d F}{d p}=\frac{K p^{l}\left(3 K-D-P D^{\prime}\right)+p^{2} K D^{\prime}}{2 \sqrt{\frac{\left(p-p^{l}\right) K}{p(3 K-D)}} p^{2}(3 K-D)^{2}}$.

We can prove that

$\frac{2 K p^{L}\left(3 K-D-P D^{\prime}\right)}{p^{2}(3 K-D)^{2}}>\frac{K p^{l}\left(3 K-D-P D^{\prime}\right)}{2 \sqrt{\frac{(p-p l) K}{p(3 K-D)}} p^{2}(3 K-D)^{2}}$ and $\frac{2 p^{2} K D^{\prime}}{p^{2}(3 K-D)^{2}}>\frac{p^{2} K D^{\prime}}{2 \sqrt{\frac{(p-p l) K}{p(3 K-D)}} p^{2}(3 K-D)^{2}}$, respectively.

To see how the first inequality holds, note that

$\frac{2 K p^{L}\left(3 K-D-P D^{\prime}\right)}{p^{2}(3 K-D)^{2}}>\frac{K p^{l}\left(3 K-D-P D^{\prime}\right)}{2 \sqrt{\frac{\left(p-p^{l}\right) K}{p(3 K-D)}} p^{2}(3 K-D)^{2}} \Leftrightarrow 4 p^{L} \sqrt{\frac{\left(p-p^{l}\right) K}{p(3 K-D)}}>p^{l}$

Because $\sqrt{\frac{\left(p-p^{l}\right) K}{p(3 K-D)}}=F>\frac{1}{2}, 4 p^{L} \sqrt{\frac{\left(p-p^{l}\right) K}{p(3 K-D)}}>2 p^{L}>p^{l}$ holds.

To see how the second inequality holds, note that

$\frac{2 p^{2} K D^{\prime}}{p^{2}(3 K-D)^{2}}>\frac{p^{2} K D^{\prime}}{2 \sqrt{\frac{\left(p-p^{l}\right) K}{p(3 K-D)}} p^{2}(3 K-D)^{2}} \Leftrightarrow 2>\frac{1}{2 \sqrt{\frac{(p-p l) K}{p(3 K-D)}}}$

which again holds true because $\sqrt{\frac{\left(p-p^{l}\right) K}{p(3 K-D)}}=F>\frac{1}{2}$.

Therefore, the slope of $F_{C}$ is everywhere (in the overlap) greater than that of $F$. If case 2 in Figure $\mathrm{A} 1$ is true, then $F_{C}$ grows so fast that it crosses $F$ at some point $p$; but in order for $F_{C}\left(p^{h}\right)<F\left(p^{h}\right)=1, F_{C}$ has to increase less slowly than $F$ for some price range, which is contradictory to the fact that $\frac{d F_{C}}{d p}>\frac{d F}{d p} \forall p \in\left(p^{L}, p^{h}\right)$. So $F_{C}$ cannot be greater that $F$ anywhere (although only part of the price range $p \in\left(p^{L}, P(2 K)\right.$ ) is applicable). Therefore, only case 1 in Figure A1 is possible, i.e., $F_{C}$ is everywhere smaller than $F$ at same price level. 
- Second, in the case where $p^{L}<P(2 K)<p<p^{H}$, the mixed strategies are $F_{M}=$ $1-\frac{p^{L}}{p}, F_{C}=\frac{p D-2 p^{L} K}{p K}$

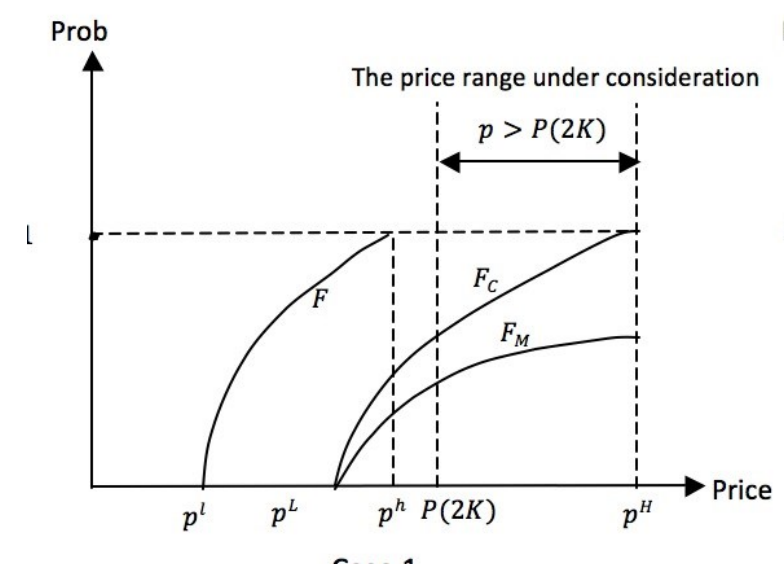

Case 1

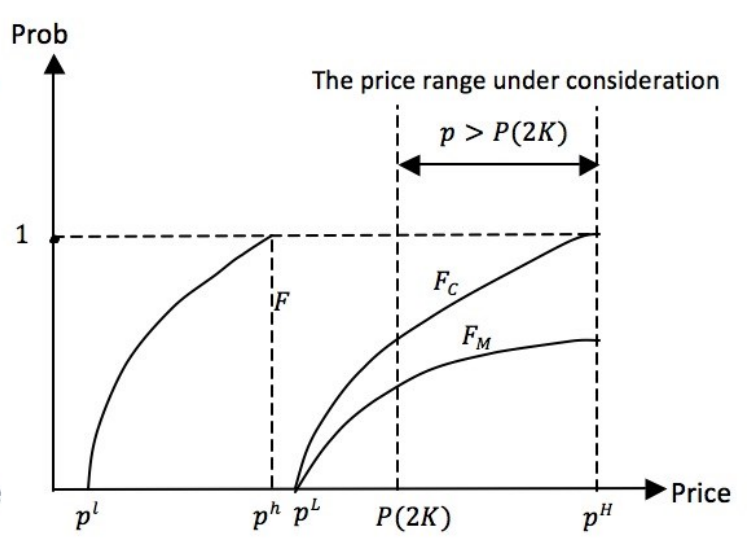

Case 2

Figure A2

There are two possibilities, as shown in Figure A2. The diagram on the left shows the case $p^{L}<p^{h}<P(2 K)<p<p^{H}$, and the diagram on the right shows the case $p^{h}<$ $p^{L}<P(2 K)<p^{H}$. In both diagrams there is no overlapping between $F$ and the relevant portion of $F_{C}$. (Because $P(2 K)<p$, the overlapping part in left figure is not relevant). Hence, for any price $p, F_{C}$ stochastically dominates $F$.

- In the case where $p^{L}>P(2 K), F_{M}=1-\frac{p^{L}}{p}, F_{C}=\frac{p D-p^{L} D^{L}}{p K}$. 


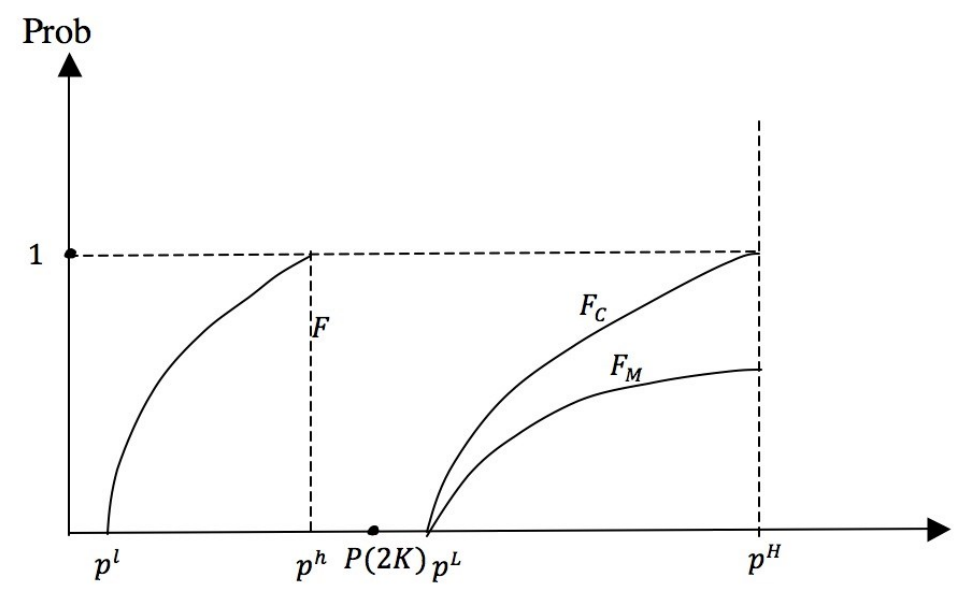

Figure A3

In this case, there is no overlapping between $F$ and $F_{C}$, as Figure A3 shows. It immediately follows that $F_{C}$ dominates $F$. 


\section{Appendix B Post-Merger Mixed Strategy Equilibrium in the Model of Section 1.5}

Here we present a detailed analysis of the post-merger equilibrium strategies in the model with a linear demand function. To be more specific, we examine the post-merger mix-strategy equilibriums, which arise if $K>a / 5 b$. Depending on the relative magnitudes of $p^{H}, p^{L}$ and $P(2 K)$, we need to consider three cases.

Case 1. If $p^{H}>P(2 K)$ and $p^{L}>P(2 K)$.

Note that $p^{H}=\frac{a-b K}{2}>P(2 K)=a-2 b K$ requires that $K \in\left(\frac{a}{3 b}, \frac{a}{2 b}\right)$. On the other hand, if $p^{L}>P(2 K)$, then firm $\mathrm{M}$ supplies the entire market demand $D^{L}$ when it plays $p^{L}: p^{L} D^{L}=p^{H}\left(D^{H}-K\right) \Leftrightarrow p^{L} \frac{a-p^{L}}{b}=\left(\frac{a-b K}{2}\right)\left(\frac{a-\frac{a-b K}{2}}{b}-K\right)=\frac{1}{4 b}(a-b K)^{2}$ $\Leftrightarrow 4 p^{L}\left(a-p^{L}\right)=(a-b K)^{2}$.

Solving the above equation, we obtain $p^{L}=\frac{a}{2} \pm \frac{1}{2} \sqrt{(2 a-b K) b K}$. The assumption $K<2 a / b$ ensures that $(2 a-b K) b K>0$. We examine each of these two roots respectively.

If $p^{L}=\frac{a}{2}+\frac{1}{2} \sqrt{(2 a-b K) b K}$, then the condition $p^{L}>P(2 K)$ requires that $\frac{a}{2}+$ $\frac{1}{2} \sqrt{(2 a-b K) b K}>a-2 b K$. Because $K<\frac{a}{2 b}$ then solve for the inequality we have $17 b^{2} K^{2}-10 a b K+a^{2}<0 \Leftrightarrow K \in\left(0.13 \frac{a}{b}, 0.46 \frac{a}{b}\right)$. So $p^{L}=\frac{a}{2}+\frac{1}{2} \sqrt{(2 a-b K) b K}$ when $K \in\left(\frac{a}{3 b}, 0.46 \frac{a}{b}\right) \cup\left(0.5 \frac{a}{b}, \frac{a}{b}\right)$ (recall that $K \in\left(\frac{a}{3 b}, \frac{a}{2 b}\right)$ if $\left.p^{H}>P(2 K)\right)$; but as we proceed to the calculation of $F_{M}$ (see below) we will have to dismiss $p^{L}=\frac{a}{2}+$ 
$\frac{1}{2} \sqrt{(2 a-b K) b K}$ as a solution. Thus, $p^{L}=\frac{a}{2}-\frac{1}{2} \sqrt{(2 a-b K) b K}$ is the only candidate solution for $p^{L}$.

Since $p^{L}=\frac{a}{2}-\frac{1}{2} \sqrt{(2 a-b K) b K}$, then the condition $p^{L}>P(2 K)$ requires that $\frac{a}{2}-\frac{1}{2} \sqrt{(2 a-b K) b K}>a-2 b K$. Solving the inequality we obtain $K \in\left(0.46 \frac{a}{b}, \frac{a}{2 b}\right)$. Hence, $p^{L}=\frac{a}{2}-\frac{1}{2} \sqrt{(2 a-b K) b K}$ if $K \in\left(0.46 \frac{a}{b}, \frac{a}{2 b}\right)$. Recall that the range of $K$ determined by $p^{H}$ is $K \in\left(\frac{a}{3 b}, \frac{a}{2 b}\right)$. Taking the intersection of these two sets, we conclude that case 1 occurs if $K \in\left(0.46 \frac{a}{b}, \frac{a}{2 b}\right)$.

Post-merger profit of firm $\mathrm{M}$ is $p^{H}\left(D^{H}-K\right)=\frac{1}{4 b}(a-b K)^{2}$, which is greater than the combined pre-merger profits of the two merging firms $2 \pi_{i}=2 p^{l} K=\frac{2}{b}\left(\frac{a}{2}-b K\right)^{2} \Leftrightarrow$ $K>0.23 \frac{a}{b}$ if and only if $K>0.23 \frac{a}{b}$. The latter is satisfied because $K \in\left(\frac{a}{3 b}, \frac{a}{2 b}\right)$. In other words, the merger is profitable for firm M. The same applies to firm C, which earns a higher profit because $p^{L}>p^{l}$.

Solving for the mixed strategy we obtain

$$
\begin{gathered}
F_{M}=1-\frac{p^{L}}{p}=1-\frac{a}{2 p} \mp \frac{1}{2 p} \sqrt{(2 a-b K) b K} ; \\
F_{C}=\frac{p D-p^{L} D^{L}}{p K}=\frac{1}{p b K}\left(a p-p^{2}-a p^{L}+p^{L^{2}}\right) .
\end{gathered}
$$

Then we see that $\left.F_{M}\right|_{p^{L}}=\left.F_{C}\right|_{p^{L}}=0,\left.\quad F_{M}\right|_{p^{H}}=1-\frac{p^{L}}{p^{H}}=1-\frac{\frac{a}{2} \pm \frac{1}{2} \sqrt{(2 a-b K) b K}}{\frac{a-b K}{2}}=1-$ $\frac{a \pm \sqrt{(2 a-b K) b K}}{a-b K}$. In order to satisfy the condition $0<\frac{a \pm \sqrt{(2 a-b K) b K}}{a-b K}<1$, we must dismiss $p^{L}=\frac{a}{2}+\frac{1}{2} \sqrt{(2 a-b K) b K}$. The other solution $p^{L}=\frac{a}{2}-\frac{1}{2} \sqrt{(2 a-b K) b K}$ satisfies the condition $0<\frac{a-\sqrt{(2 a-b K) b K}}{a-b K}<1$ because $K<\frac{a}{b}$. We learn from these that $\left.F_{M}\right|_{p^{H}}<1$, 
which means firm $\mathrm{M}$ 's mixed strategy involves a positive probability at supremum $p^{H}$.

On the other hand, $\left.F_{C}\right|_{p^{H}}=\frac{p^{H} D^{H}-p^{L} D^{L}}{p^{H} K}=\frac{p^{H} K}{p^{H} K}=1\left(\right.$ recall that $\left.p^{L} D^{L}=p^{H}\left(D^{H}-K\right)\right)$.

Case 2. If $p^{H}<P(2 K)$ (and hence $p^{L}<P(2 K)$ )

$$
\text { If } p^{H}=\frac{a-b K}{2} \leq P(2 K)=a-2 b K \text {, then } K \in\left(\frac{a}{5 b}, \frac{a}{3 b}\right) \text {. Firm M sells to the capacity }
$$

$2 K$ when it undercuts its rival. Hence,

$$
p^{L}=\frac{p^{H}\left(D^{H}-K\right)}{2 K}=\frac{1}{2 K}\left(\frac{a-b K}{2}\right)\left(\frac{a-\frac{a-b K}{2}}{b}-K\right)=\frac{1}{2 b K}\left(\frac{a-b K}{2}\right)^{2}=\frac{1}{8 b K}(a-b K)^{2} .
$$

To ensure that $p^{L}<P(2 K)=a-2 b K$, we need $17 b^{2} K^{2}-10 a b K+a^{2}<0 \Leftrightarrow K \in$ $\left(0.13 \frac{a}{b}, 0.46 \frac{a}{b}\right)$. Now $p^{l}-p^{L}=\frac{1}{8 b K}\left(7 b^{2} K^{2}-6 a b K+a^{2}\right)$, which is negative when $K \in\left(0.23 \frac{a}{b}, 0.63 \frac{a}{b}\right)$. Recall that when we compare $p^{L}$ with $p^{l}$, we also require that $K>\frac{a}{4 b}$ (the threshold of pre-merger mixed equilibrium) and $K \in\left(\frac{a}{5 b}, \frac{a}{3 b}\right)$ (to ensure $\left.p^{H} \leq P(2 K)\right) ;$ so $K$ must belong to $\left(0.25 \frac{a}{b}, 0.33 \frac{a}{b}\right)$, which is a subset of $\left(0.23 \frac{a}{b}, 0.63 \frac{a}{b}\right)$. Hence, $p^{l}<p^{L}$.

Firm $\mathrm{M}$ earns an expected profit $2 K p^{L}=\frac{1}{4 b}(a-b K)^{2}$, which, as shown in Case 1 , is greater than $2 p^{l} K=\frac{2}{b}\left(\frac{a}{2}-b K\right)^{2}$. Similarly, firm $\mathrm{C}$ earns a higher profit than before the merger.

Lastly, we proceed to check the properties of mixed strategies $F_{M}$ and $F_{C}$

$$
F_{M}=\frac{\left(p-p^{L}\right) K}{p(3 K-D)}=\frac{8 b p K-(a-b K)^{2}}{8 p(3 b K-a+p)}, \quad F_{C}=\frac{2\left(p-p^{L}\right) K}{p(3 K-D)}=\frac{8 b p K-(a-b K)^{2}}{4 p(3 b K-a+p)}
$$

We see that $\left.F_{M}\right|_{p^{L}}=\left.F_{C}\right|_{p^{L}}=0$, and that

$$
\left.F_{M}\right|_{p^{H}}=\frac{\left(p^{H}-p^{L}\right) K}{p^{H}\left(3 K-D^{H}\right)}=\frac{\left(\frac{a-b K}{2}-\frac{1}{8 b K}(a-b K)^{2}\right) K}{\frac{a-b K}{2}\left(3 K-\frac{a-\frac{a-b K}{2}}{b}\right)}=\frac{5 b K-a}{10 b K-2 a}=\frac{1}{2}
$$


The latter means firm $\mathrm{M}$ will play $p^{H}$ with probability of $\frac{1}{2}$. In addition,

$$
\left.F_{C}\right|_{p^{H}}=\frac{2\left(p^{H}-p^{L}\right) K}{p^{H}\left(3 K-D^{H}\right)}=\frac{3 p^{H} K-p^{H} D^{H}}{p^{H}\left(3 K-D^{H}\right)}=1
$$

In this case, $F_{C}$ and $F$ may overlap (when $K \in\left(0.25 \frac{a}{b}, 0.46 \frac{a}{b}\right)$ ). Since we have shown in the general demand function case in section 4 that $F$ dominates $F_{C}$, we do not repeat the analysis here.

Case 3. If $p^{H}>P(2 K)$ and $p^{L}<P(2 K)$

From the analysis of the first two cases, we have learned that $p^{H}=\frac{a-b K}{2}>P(2 K)=$ $a-2 b K$ requires that $K \in\left(\frac{a}{3 b}, \frac{a}{2 b}\right)$, and that $p^{L}=\frac{1}{8 b K}(a-b K)^{2}<P(2 K)$ requires that $K \in\left(0.13 \frac{a}{b}, 0.46 \frac{a}{b}\right)$. Hence, the relevant range of $K$ for case 3 is $K \in\left(\frac{a}{3 b}, 0.46 \frac{a}{b}\right)$. Since $\left(\frac{a}{3 b}, 0.46 \frac{a}{b}\right) \subset\left(0.23 \frac{a}{b}, 0.63 \frac{a}{b}\right)$, we know from the results in Case 2 that $p^{l}<p^{L}$.

We have to consider $p$ in two separate intervals, $\left(p^{L}, P(2 K)\right)$ and $\left(P(2 K), p^{H}\right)$. If $p^{L}<p<P(2 K)$, the relevant portion of the mixed strategies is represented by

$$
F_{M}=\frac{\left(p-p^{L}\right) K}{p(3 K-D)}=\frac{8 b p K-(a-b K)^{2}}{8 p(3 b K-a+p)}, \text { and } F_{C}=\frac{2\left(p-p^{L}\right) K}{p(3 K-D)}=\frac{8 b p K-(a-b K)^{2}}{4 p(3 b K-a+p)}
$$

where $\left.F_{M}\right|_{p^{L}},\left.F_{C}\right|_{p^{L}}$ are the same as in Case 2. But $\left.F_{M}\right|_{p^{H}},\left.F_{C}\right|_{p^{H}}$ do not apply here because $p<P(2 K)$ but $p^{H}>P(2 K)$. In this situation $F_{C}$ and $F$ may overlap, but since $F_{C}$ has the same expression as in Case 2, we do not need to compare them here where they overlap.

If $P(2 K)<p<p^{H}$, the relevant portion of the mixed strategies are

$$
F_{M}=1-\frac{p^{L}}{p}=1-\frac{1}{8 b p K}(a-b K)^{2}, F_{C}=\frac{p D-2 p^{L} K}{p K}=\frac{4 p(a-p)-(a-b K)^{2}}{4 b p K} .
$$


Then $\left.F_{M}\right|_{p^{H}}=1-\frac{p^{L}}{p^{H}}<1$, which means firm $\mathrm{M}$ will play $p^{H}$ with positive probability while $\left.F_{C}\right|_{p^{H}}=\frac{p^{H} D^{H}-2 p^{L} K}{p^{H} K}=1$ because $p^{H}\left(D^{H}-K\right)=2 p^{L} K$. In this situation $F_{C}$ and $F$ do not overlap, so there is no need to compare them.

Firm M earns a profit $2 K p^{L}=\frac{1}{4 b}(a-b K)^{2}$ which, as has been shown in Case 1 , is greater than the combined profits of two firms before the merger, $2 p^{l} K=\frac{2}{b}\left(\frac{a}{2}-b K\right)^{2}$. Similarly, firm $\mathrm{C}$ earns a higher profit than before the merger. 


\section{Appendix C Post-Merger Mixed Strategy Equilibriums in the Model of Section 1.6}

Here we characterize the post-merger mixed-strategy of firm $M$ and each outside firm, denoted by $F_{M}$ and $F_{C}$, respectively. Depending on the position of $P((n-1) K)$ relative to $p^{L}$ and $p^{H}$, there are three cases to consider.

- If $p^{L}<P((n-1) K)<p^{H}$, the expected profit of firm $\mathrm{M}$ can be written as

$$
\begin{aligned}
& \pi_{M}\left(F_{M}, F_{C}\right)=\int_{p^{L}}^{p^{H}} \pi_{M}\left(p_{M}, F_{C}\right) d F_{M} \\
& =\int_{p^{L}}^{P((n-1) K)}\left[F^{n-2} p(D-(n-2) K)+\left(1-F_{C}^{n-2}\right) p 2 K\right] d F_{M} \\
& +\int_{P((n-1) K)}^{p^{H}}\left[F_{C}^{n-2} p(D-(n-2) K)+(n-2) F_{C}^{n-3}(1-F) p(D-(n-3) K)+\right. \\
& \left.\left(1-F_{C}^{n-2}-(n-2) F^{n-3}\left(1-F_{C}\right)\right) p 2 K\right] d F_{M}=2 p^{L} K,
\end{aligned}
$$

where $F_{C}^{n-2}$ is the probability that firm $\mathrm{M}$ offers the highest price, and $(n-$ 2) $F_{C}^{n-3}(1-F)$ is the probability that it offers second highest price. On the other hand, the expected profit of an outside firm is

$$
\begin{aligned}
& \pi_{C}\left(F_{M}, F_{C}\right)=\int_{p^{L}}^{p^{H}} \pi_{C}\left(p_{C}, F_{M}\right) d F_{C} \\
= & \int_{p^{L}}^{P((n-1) K)}\left[F_{M} F_{C}^{n-3} p(D-(n-1) K)+\left(1-F_{M} F_{C}^{n-3}\right) p K\right] d F_{C} \\
+ & \int_{P((n-1) K)}^{p^{H}}\left[F_{M} F_{C}^{n-3} p \times 0+(n-3) F_{M} F^{n-4}(1-F) p(D-(n-2) K)\right. \\
+ & \left.\left(1-F_{M} F_{C}^{n-3}-(n-3) F_{M} F_{C}^{n-4}(1-F)\right) p K\right] d F_{C}=p^{L} K,
\end{aligned}
$$

where $F_{M} F_{C}^{n-3}$ is the probability that this outside firm offers the highest price, and $(n-3) F_{M} F_{C}^{n-4}\left(1-F_{C}\right)$ is the probability that this firm offers the second highest price and $\mathrm{M}$ offers an even lower price.

- If $p^{H}<P((n-1) K)$, we have 


$$
\begin{aligned}
& \pi_{M}\left(F_{M}, F_{C}\right)=\int_{p^{L}}^{p^{H}} \pi_{M}\left(p_{M}, F_{C}\right) d F_{M}= \\
& \int_{p^{L}}^{p^{H}}\left[F_{C}^{n-2} p(D-(n-2) K)+\left(1-F_{C}^{n-2}\right) p 2 K\right] d F_{M}=2 p^{L} K \\
& \pi_{C}\left(F_{M}, F_{C}\right)=\int_{p^{L}}^{p^{H}} \pi_{C}\left(p_{C}, F_{M}\right) d F_{C} \\
& =\int_{p^{L}}^{p^{H}}\left[F_{M} F_{C}^{n-3} p(D-(n-1) K)+\left(1-F_{M} F_{C}^{n-3}\right) p K\right] d F_{C}=p^{L} K .
\end{aligned}
$$

- If $p^{L}>P((n-1) K)$, we have

$$
\begin{aligned}
& \pi_{M}\left(F_{M}, F_{C}\right)=\int_{p^{L}}^{p^{H}} \pi_{M}\left(p_{M}, F_{C}\right) d F_{M} \\
& =\int_{p^{L}}^{p^{H}}\left[F_{C}^{n-2} p(D-(n-2) K)+(n-2) F_{C}^{n-3}(1-F) p(D-(n-3) K)\right. \\
& \left.+\left(1-F_{C}^{n-2}-(n-2) F_{C}^{n-3}\left(1-F_{C}\right)\right) p 2 K\right] d F_{M}=p^{L} 2 K \\
& \pi_{C}\left(F_{M}, F_{C}\right)=\int_{p^{L}}^{p^{H}} \pi_{C}\left(p_{C}, F_{M}\right) d F_{C} \\
& =\int_{p^{L}}^{p^{H}}\left[F_{M} F_{C}^{n-3} p \times 0+(n-3) F_{M} F_{C}^{n-4}\left(1-F_{C}\right) p(D-(n-2) K)\right. \\
& \left.+\left(1-F_{M} F_{C}^{n-3}-(n-3) F_{M} F_{C}^{n-4}\left(1-F_{C}\right)\right) p K\right] d F_{C}=p^{L} K .
\end{aligned}
$$

From the above we are able to derive (1.12) as the solution for $F_{M}$ and $F_{C}$ for the case where $p^{H}<P((n-1) K)$.

In the case $p^{L}<P((n-1) K)<p^{H}, F_{M}$ and $F_{C}$ are the same as (1.12) for prices in the range $p^{L}<p<P((n-1) K)$. However, because the firms' expected profits are in the form of high-degree polynomials in $F$, we are not able to obtain an explicit solution of $F$ for prices in the range $P((n-1) K)<p<p^{H}$ or for the case $p^{L}>P((n-1) K)$.

Finally, we prove that the post-merger price distributions $F_{M}$ and $F_{C}$ stochastically dominate the pre-merger price distribution $F=\left(\frac{\left(p-p^{l}\right) K}{p(n K-D)}\right)^{\frac{1}{n-1}}$. For any price below $p^{L}$ or above $p^{h}$, it is self-evident that $F_{M}$ and $F_{C}$ stochastically dominate $F$. Hence, the key part 
of this proof is to show that for any price in the range $p^{L}<p<p^{h}<P((n-1) K)$, we have $F>F_{C}$. It can be seen from (1.12) that $F_{C}>F_{M}$ for prices in the same range. Therefore, we can conclude that $F>F_{M}$ once we establish that $F>F_{C}$.

The slopes of mixed strategies $F$ and $F_{C}$ are given by

$$
\begin{aligned}
& \frac{d F}{d p}=\frac{1}{n-1}\left(\frac{\left(p-p^{l}\right) K}{p(n K-D)}\right)^{\frac{1}{n-1}-1} \frac{K p(n K-D)-\left(p-p^{l}\right) K\left(n K-D-p D^{\prime}\right)}{p^{2}(n K-D)^{2}}=\frac{1}{n-1}\left(\frac{\left(p-p^{l}\right) K}{p(n K-D)}\right)^{-\frac{n-2}{n-1}} \frac{K p(n K-D)-\left(p-p^{l}\right) K\left(n K-D-p D^{\prime}\right)}{p^{2}(n K-D)^{2}} \\
& \frac{d F_{C}}{d p}=\frac{1}{n-2}\left(\frac{2\left(p-p^{L}\right) K}{p(n K-D)}\right)^{\frac{1}{n-2}-1} \frac{2 K p(n K-D)-2\left(p-p^{L}\right) K\left(n K-D-p D^{\prime}\right)}{p^{2}(n K-D)^{2}}=\frac{2}{n-2}\left(\frac{2\left(p-p^{L}\right) K}{p(n K-D)}\right)^{-\frac{n-3}{n-2}} \frac{K p(n K-D)-\left(p-p^{L}\right) K\left(n K-D-p D^{\prime}\right)}{p^{2}(n K-D)^{2}}
\end{aligned}
$$

We show below that, for any common $p$, each term in $\frac{d F_{C}}{d p}$ is greater than the corresponding term in $\frac{d F}{d p}$. First, it is obvious that $\frac{2}{n-2}>\frac{1}{n-1}$. Second, $\frac{K p(n K-D)-\left(p-p^{L}\right) K\left(n K-D-p D^{\prime}\right)}{p^{2}(n K-D)^{2}}>\frac{K p(n K-D)-\left(p-p^{l}\right) K\left(n K-D-p D^{\prime}\right)}{p^{2}(n K-D)^{2}}$.

Finally, $\left(\frac{2\left(p-p^{L}\right) K}{p(n K-D)}\right)^{\frac{1}{n-2}-1}=\left(\frac{2\left(p-p^{L}\right) K}{p(n K-D)}\right)^{-\frac{n-3}{n-2}}>\left(\frac{\left(p-p^{l}\right) K}{p(n K-D)}\right)^{\frac{1}{n-1}-1}=\left(\frac{\left(p-p^{l}\right) K}{p(n K-D)}\right)^{-\frac{n-2}{n-1}}$. The critical part of this inequality is the fact that $p-p^{l}>2\left(p-p^{L}\right)$. The latter can be proved by noting that $F_{C}\left(p^{h}\right)<F\left(p^{h}\right)=1$ implies $\left(\frac{\left(p^{h}-p^{l}\right) K}{p(n K-D)}\right)^{\frac{1}{n-1}}>\left(\frac{2\left(p^{h}-p^{L}\right) K}{p(n K-D)}\right)^{\frac{1}{n-2}}$. Then $p^{h}-p^{l}>2\left(p^{h}-p^{L}\right)$ because $\frac{1}{n-1}<\frac{1}{n-2}$. Note that the difference $p^{h}-p^{l}-$ $2\left(p^{h}-p^{L}\right)$ is inversely correlated to $p^{h}$; so as the price falls from $p^{h}$, this gap grows. Hence, for any $p \in\left(p^{L}, p^{h}\right)$, we must have $p-p^{l}>2\left(p-p^{L}\right)$.

The analysis in the preceding paragraph establishes that $\frac{d F_{C}}{d p}>\frac{d}{d p}$ for all $p \in$ $\left(p^{L}, p^{h}\right)$. Then give the fact that $F>F_{C}$ both at points $p^{L}$ and $p^{h}$, we can conclude that $F>F_{C} \forall$ all $p \in\left(p^{L}, p^{h}\right)$, and thus $F_{M}$ and $F_{C}$ stochastically dominate $F$ in the case $p^{H}<P((n-1) K)$. 


\section{Appendix D Merger Simulation ${ }^{55}$}

\section{Solutions to the Equilibrium}

The demand system is

$$
\begin{aligned}
& \left(\begin{array}{c}
p_{1} \\
\vdots \\
\vdots \\
p_{i} \\
\vdots \\
p_{N}
\end{array}\right)=\left(\begin{array}{c}
\alpha \\
\vdots \\
\vdots \\
\vdots \\
\vdots \\
\alpha
\end{array}\right)-\left(\begin{array}{cccccc}
\beta & \gamma & \delta & \cdots & \cdots & \delta \\
\gamma & \beta & \delta & \cdots & \cdots & \delta \\
\varphi & \varphi & \beta & \varphi & \cdots & \varphi \\
\vdots & \vdots & \varphi & \ddots & \varphi & \vdots \\
\vdots & \vdots & \vdots & \varphi & \beta & \varphi \\
\varphi & \cdots & \cdots & \cdots & \varphi & \beta
\end{array}\right)_{N \times N}\left(\begin{array}{c}
q_{1} \\
\vdots \\
\vdots \\
q_{i} \\
\vdots \\
q_{N}
\end{array}\right) \text { or, } \boldsymbol{p}=\boldsymbol{\alpha}-\boldsymbol{A q} \\
& \text { where } \boldsymbol{A}=\left(\begin{array}{cccccc}
\beta & \gamma & \delta & \cdots & \cdots & \delta \\
\gamma & \beta & \delta & \cdots & \cdots & \delta \\
\varphi & \varphi & \beta & \varphi & \cdots & \varphi \\
\vdots & \vdots & \varphi & \ddots & \varphi & \vdots \\
\vdots & \vdots & \vdots & \varphi & \beta & \varphi \\
\varphi & \cdots & \cdots & \cdots & \varphi & \beta
\end{array}\right)_{N \times N}
\end{aligned}
$$

The system of F.O.C before the merger is

$$
\begin{aligned}
& \left(\begin{array}{c}
\alpha-c \\
\vdots \\
\vdots \\
\vdots \\
\vdots \\
\alpha-c
\end{array}\right)=\left(\begin{array}{cccccc}
2 \beta & \gamma & \delta & \cdots & \cdots & \delta \\
\gamma & 2 \beta & \delta & \cdots & \cdots & \delta \\
\varphi & \varphi & 2 \beta & \varphi & \cdots & \varphi \\
\vdots & \vdots & \varphi & \ddots & \varphi & \vdots \\
\vdots & \vdots & \vdots & \varphi & 2 \beta & \varphi \\
\varphi & \cdots & \cdots & \cdots & \varphi & 2 \beta
\end{array}\right)_{N \times N}\left(\begin{array}{c}
q_{1}^{*} \\
\vdots \\
\vdots \\
q_{i}^{*} \\
\vdots \\
q_{N}^{*}
\end{array}\right) \text { or }, \\
& \alpha-\boldsymbol{c}=\left(\boldsymbol{A}+\beta \boldsymbol{I}_{N \times N}\right) \boldsymbol{q}^{*} .
\end{aligned}
$$

We combine the demand and F.O.C. system as

$$
\left(\begin{array}{cc}
\boldsymbol{I}_{N \times N} & \boldsymbol{A} \\
\boldsymbol{O}_{N \times N} & \boldsymbol{A}+\beta \boldsymbol{I}
\end{array}\right)\left(\begin{array}{l}
\boldsymbol{p}^{*} \\
\boldsymbol{q}^{*}
\end{array}\right)=\left(\begin{array}{cc}
\boldsymbol{I}_{N \times N} & \boldsymbol{O}_{N \times N} \\
\boldsymbol{I}_{N \times N} & -\boldsymbol{I}_{N \times N}
\end{array}\right)\left(\begin{array}{l}
\boldsymbol{\alpha} \\
\boldsymbol{c}
\end{array}\right),
$$

The pre-merger equilibrium is, therefore,

$$
\left(\begin{array}{l}
\boldsymbol{p}^{*} \\
\boldsymbol{q}^{*}
\end{array}\right)=\left(\begin{array}{cc}
\boldsymbol{I}_{N \times N} & \boldsymbol{A} \\
\boldsymbol{O}_{N \times N} & \boldsymbol{A}+\beta \boldsymbol{I}_{N \times N}
\end{array}\right)^{-1}\left(\begin{array}{cc}
\boldsymbol{I}_{N \times N} & \boldsymbol{O}_{N \times N} \\
\boldsymbol{I}_{N \times N} & -\boldsymbol{I}_{N \times N}
\end{array}\right)\left(\begin{array}{l}
\boldsymbol{\alpha} \\
\boldsymbol{c}
\end{array}\right) .
$$

The system of F.O.C. after the merger is

\footnotetext{
${ }^{55}$ In Appendix D, we assume the slope of a firm's demand curve in its own quantity is $\beta$ rather than 1, e.g., $p_{1}=\alpha-\beta q_{1}-\gamma q_{2}-\delta \sum_{j=3}^{N} q_{j}$ and so on. We will let $\beta=1$ in the calculation.
} 


$$
\begin{aligned}
& \left(\begin{array}{c}
\alpha-(1-E) c \\
\alpha-(1-E) c \\
\alpha-c \\
\vdots \\
\vdots \\
\alpha-c
\end{array}\right)=\left(\begin{array}{cccccc}
2 \beta & 2 \gamma & \delta & \cdots & \cdots & \delta \\
2 \gamma & 2 \beta & \delta & \cdots & \cdots & \delta \\
\varphi & \varphi & 2 \beta & \varphi & \cdots & \varphi \\
\vdots & \vdots & \varphi & \ddots & \varphi & \vdots \\
\vdots & \vdots & \vdots & \varphi & 2 \beta & \varphi \\
\varphi & \cdots & \cdots & \cdots & \varphi & 2 \beta
\end{array}\right)_{N \times N}\left(\begin{array}{c}
q_{1}^{e} \\
\vdots \\
\vdots \\
q_{i}^{e} \\
\vdots \\
q_{N}^{e}
\end{array}\right) \text { or }, \\
& \boldsymbol{\alpha}-\boldsymbol{E} \boldsymbol{c}=\boldsymbol{B} \boldsymbol{q}^{\boldsymbol{e}} \text {, where } \boldsymbol{B}=\left(\begin{array}{cccccc}
2 \beta & 2 \gamma & \delta & \cdots & \cdots & \delta \\
2 \gamma & 2 \beta & \delta & \cdots & \cdots & \delta \\
\varphi & \varphi & 2 \beta & \varphi & \cdots & \varphi \\
\vdots & \vdots & \varphi & \ddots & \varphi & \vdots \\
\vdots & \vdots & \vdots & \varphi & 2 \beta & \varphi \\
\varphi & \cdots & \cdots & \cdots & \varphi & 2 \beta
\end{array}\right)_{N \times N} \text { and } \\
& \boldsymbol{E}=\left(\begin{array}{ccccc}
1-E & & & & \boldsymbol{o} \\
& 1-E & & & \\
& & 1 & \ddots & \\
\boldsymbol{o} & & & & 1
\end{array}\right)_{N \times N} .
\end{aligned}
$$

We combine the demand and F.O.C. system as

$$
\left(\begin{array}{cc}
\boldsymbol{I}_{N \times N} & \boldsymbol{A} \\
\boldsymbol{O}_{N \times N} & \boldsymbol{B}
\end{array}\right)\left(\begin{array}{c}
\boldsymbol{p}^{\boldsymbol{e}} \\
\boldsymbol{q}^{\boldsymbol{e}}
\end{array}\right)=\left(\begin{array}{cc}
\boldsymbol{I}_{N \times N} & \boldsymbol{O}_{N \times N} \\
\boldsymbol{I}_{N \times N} & -\boldsymbol{I}_{N \times N}
\end{array}\right)\left(\begin{array}{c}
\boldsymbol{\alpha} \\
\boldsymbol{E} \boldsymbol{c}
\end{array}\right) \text {, and }
$$

the post-merger equilibrium is $\left(\begin{array}{c}\boldsymbol{p}^{\boldsymbol{e}} \\ \boldsymbol{q}^{\boldsymbol{e}}\end{array}\right)=\left(\begin{array}{cc}\boldsymbol{I}_{N \times N} & \boldsymbol{A} \\ \boldsymbol{O}_{N \times N} & \boldsymbol{B}\end{array}\right)^{-1}\left(\begin{array}{cc}\boldsymbol{I}_{N \times N} & \boldsymbol{O}_{N \times N} \\ \boldsymbol{I}_{N \times N} & -\boldsymbol{I}_{N \times N}\end{array}\right)\left(\begin{array}{c}\boldsymbol{\alpha} \\ \boldsymbol{E} \boldsymbol{c}\end{array}\right)$.

\section{Matlab Codes for Premerger Equilibrium:}

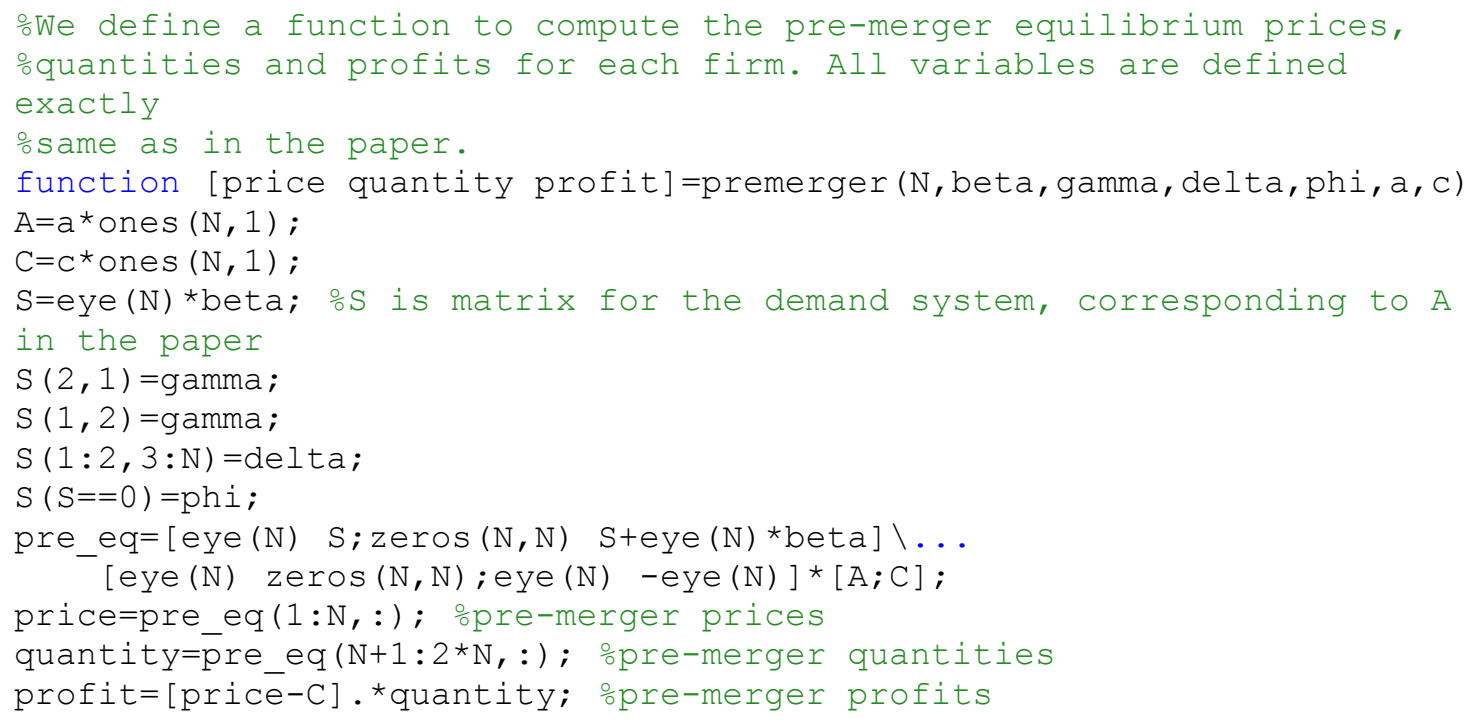




\section{Matlab Codes for Post-merger Equilibrium:}

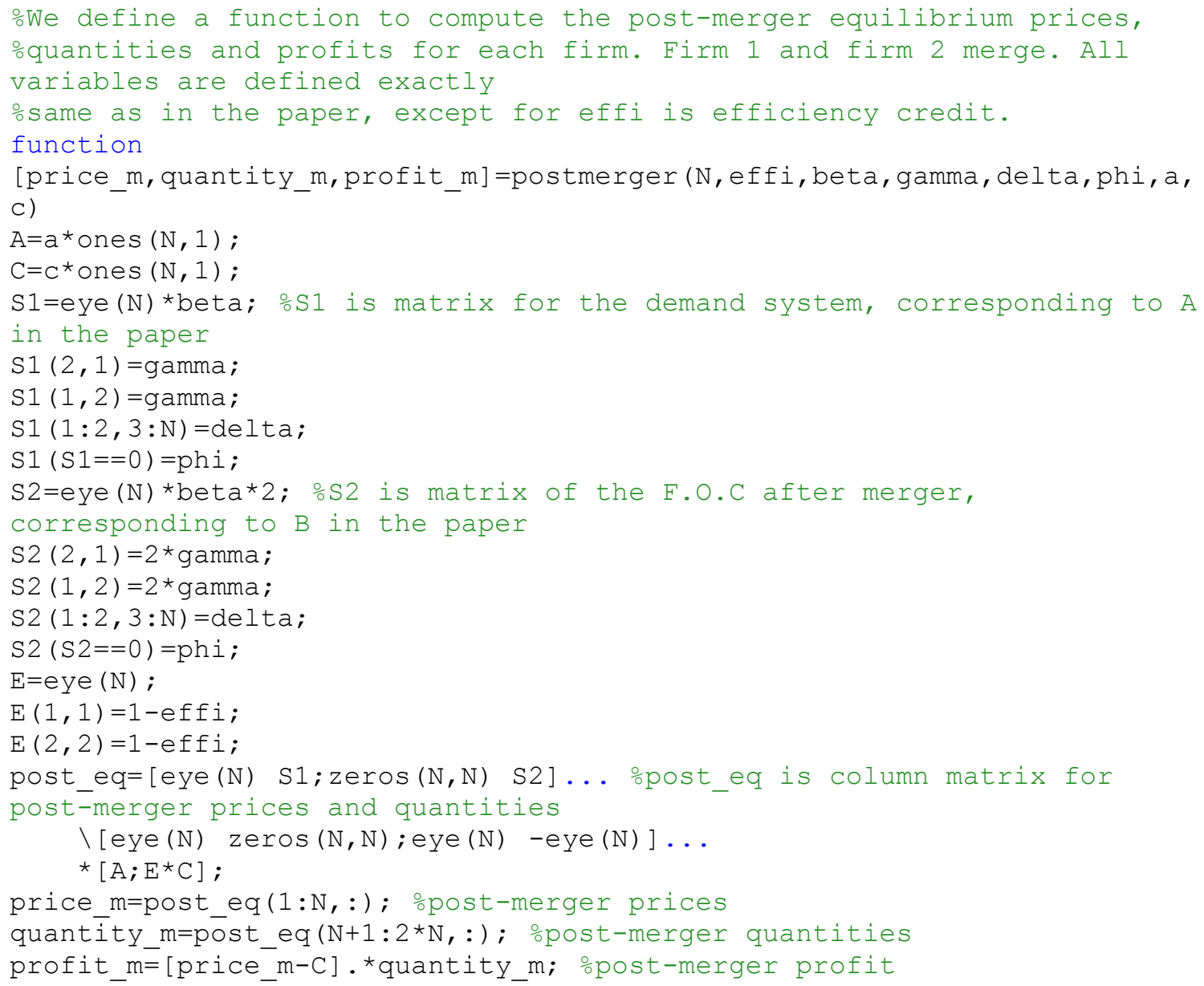




\section{References}

Allen, B. and M. Hellwig (1986). "Bertrand-Edgeworth Oligopoly in Large Markets." The Review of Economic Studies 52(2): 175-204.

Baker, J. B. and T. F. Bresnahan (1985). "The Gains from Merger or Collusion in Product-Differentiated Industries." The Journal of Industrial Economics 33(4): 427444.

Baker, J. B. (1997). "Product Differentiation Through Space and Time: Some Antitrust Policy Issues Economics." Antitrust bulletin 42: 177-196.

Baye, M. and G. Hunter (2010). " Going Beyond the Conventional Wisdom on Whether Merger-Related Cost Savings Will Benefit Customers. " Antitrust Insights Spring.

Bian, L. and D. G. McFetridge (2000). "The Efficiencies Defence in Merger Cases: Implications of Alternative Standards." The Canadian Journal of Economics 33(2): 297-318.

Braid, R. M. (1986). "Stackelberg price leadership in spatial competition." International Journal of Industrial Organization 4(4): 439-449.

Braid, R. M. (1999). "The price and profit effects of horizontal mergers in twodimensional spatial competition." Economics Letters 62: 113-119.

Cabral, L. (2003). " Horizontal mergers with free-entry: why cost efficiencies may be a weak defense and asset sales a poor remedy." International Journal of Industrial Organization 21(5): 607-623.

Cheung, F. K. (1992). "Two remarks on the equilibrium analysis of horizontal merger " Economics Letters 40(1): 119-123.

Cheviakov, A. and J. M. Hartwick (2005). "Beckmann's Edgeworth-Bertrand Duopoly Example Revisited." International Game Theory Review 7(4): 461-472.

Choné, P. and L. Linnemer (2010). A Treatment Effect Method for Merger Analysis with an Application to Parking Prices in Paris. $\underline{\text { CESifo Working Paper. }}$

Dasgupta, P. and E. Maskin (1986a). "The Existence of Equilibrium in Discontinuous Economic Games, I: Theory." The Review of Economic Studies 53(1): 1-26.

Dasgupta, P. and E. Maskin (1986b). "The Existence of Equilibrium in Discontinuous Economic Games, II: Applications." The Review of Economic Studies 53(1): 27-41. 
Daughety, A. F. (1990). "Beneficial Concentration." The American Economic Review 80(5): 1231-1237.

Davis, D. and B. Wilson (2006). "Equilibrium Price Dispersion, Mergers and Synergies: An Experimental Investigation of Differentiated Product Competition." International Journal of the Economics of Business 13(2):169-194.

Davidson, C. and A. Mukherjee (2007). "Horizontal mergers with free entry." International Journal of Industrial Organization 25(1): 157 - 172.

Davidson, C. and R. Deneckere (1984). " Horizontal mergers and collusive behavior." International Journal of Industrial Organization 2(2): 117-132.

Deneckere, R. and C. Davidson (1985). "Incentives to Form Coalitions with Bertrand Competition" The RAND Journal of Economics 16(4): 473-486

DOJ and FTC (1982/84, 1997, 2010). "Horizontal Merger Guidelines."

European Commission (2004). "Guidelines on the assessment of horizontal mergers under the Council Regulation on the control of concentrations between undertakings." European Commission Official Journal C31: 5-18.

Ebina, T. and D. Shimizu (2009). "Sequential Mergers With Differing Differentiation Levels." Australian Economic Papers 48(3): 237-251.

Erkal, N. and D. Piccinin (2010). "Welfare-Reducing Mergers in Differentiated Oligopolies with Free Entry." Economic Record 86(273): 178-184.

Farrell, J. and C. Shapiro (1990). "Horizontal Mergers: An Equilibrium Analysis." The American Economic Review 80(1): 20.

Farrell, J. and C. Shapiro (2010). "Antitrust Evaluation of Horizontal Mergers: An Economic Alternative to Market Definition." The B.E. Journal of Theoretical Economics-Policies and Perspectives 10(1): Article 9

Faulí-Oller, R. (1997). "On Merger Profitability In A Cournot Setting." Economics Letters 54(1): 75-79.

Fisher, A. A., F. I. Johnson and R. H. Lande (1989). " Price Effects of Horizontal Mergers." California Law Review 77(4): 777-827.

Francesco, M. A. D. and N. Salvadori (2009). "Bertrand-Edgeworth games under oligopoly with a complete characterization for the triopoly." MPRA Paper No. 24087. 
Francesco, M. A. D. and N. Salvadori (2010). "Bertrand-Edgeworth competition in an almost symmetric oligopoly." MPRA Paper No. 24228.

Froeb, L., S. Tschantz, and P. Crooke (2003). "Bertrand competition with capacity constraints: mergers among parking lots." Journal of Econometrics 113(1): 49 - 67.

Gandhi, A., L. Froeb, S. Tschantz, G. J. Werden (2008). "Post-Merger Product Repositioning." Journal of Industrial Economics 56(1): 49-67.

Gillen, D. and B. Martin (2009). "Price Volatility in the Airline Markets." Transportation Research Part E 45(5): 693-709.

Hausman, J., G. Leonard and J. D. Zona (1994). "Competitive Analysis with Differenciated Products." Annals of Economics and Statistics / Annales d'Économie et de Statistique 34: 159-180.

Heywood, J. S. and M. McGinty (2007). "Convex costs and the merger paradox revisited." Economic Inquiry 45(2): 342-349.

Higgins, R. S., P. A. Johnson, J.T. Sullivan (2004). "Spatial Competition and Merger." The B.E. Journals in Economic Analysis \& Policy-Topics 4(1).

Hirata, D. (2009). "Asymmetric Bertrand-Edgeworth Oligopoly and Mergers." The B.E. Journal of Theoretical Economics Bulletin 9(1): Article 22.

Hsu, J. and X. H. Wang (2010). "Horizontal Mergers In A Differentiated Cournot Oligopoly." Bulletin of Economic Research 62(3): 305-314.

Kate, A. T. and G. Niels (2005). "To What Extent are Cost Savings Passed on to Consumers? An Oligopoly Approach." European Journal of Law and Economics 20(3): 323-337.

Kim, E. H. and V. Singal (1993). "Mergers and market power: Evidence from the airline industry ." The American Economic Review 83(3): 549-569.

Kreps, D. M. and J. A. Scheinkman (1983). "Quantity Precommitment and Bertrand Competition Yield Cournot OutcomesAuthor." The Bell Journal of Economics 14(2): 326-337.

Levitan, R. and M. Shubik (1972). "Price Duopoly and Capacity Constraints." International Economic Review 13(1): 111-122. 
Levy, D. T. and J. D. Reitzes (1992). "The Anticompetitive Effects of Mergers in Markets with Localized Competition." The Journal of Law, Economics, \& Organization 8(2): 427-440.

Levy, D. T. and J. D. Reitzes (1993). "Product differentiation and the ability to collude: Where being different can be an advantage." Antitrust Bulletin 38(2): 349-368.

Lommerud, K. E. and L. Sørgard (1997). "Merger and product range rivalry." International Journal of Industrial Organization 16(1): 21-42.

Matsushima, N. (2001). "Horizontal Mergers and Merger Waves in a Location Model." Australian Economic Papers 40(3): 263-286.

McAfee, R. P., J. J. Simons, and M.A. Williams (1992). "Horizontal Mergers in Spatially Differentiated Noncooperative Markets." The Journal of Industrial Economics 40(4): 349-358.

McAfee, R. P. and M. A. Williams (1992). "Horizontal Mergers and Antitrust Policy." The Journal of Industrial Economics 40(2): 181-187.

M'Chirgui, Z. and W. Hichri (2006). "Horizontal merger in bilaterally duopolistic industries with differentiated products." Applied Economics Letters 13(2): 93-95.

Moresi, S. (2009). "Cournot Competition and The UPP Test. " HMG Review ProjectComment. Project No. P092900.

Motta, M. (2004). "Competition Policy. Theory and Practice." Cambridge University Press.

Motta, M. and H. Vasconcelos (2005). "Efficiency gains and myopic antitrust authority in a dynamic merger game." International Journal of Industrial Organization 23(9-10): $777-801$.

Norman, G. and L. Pepall (2000). "Profitable mergers in a Cournot model of spatial competition." Southern Economic Journal 66(3): 667-681.

Osborne, M. J. and C. Pitchik (1986). "Price competition in a capacity-constrained duopoly " Journal of Economic Theory 38(2): 238-260.

Perry, M. K. and R. H. Porter (1985). "Oligopoly and the Incentive for Horizontal Merger." The American Economic Review 75(1): 219-227.

Reitzes, J. D. and D. T. Levy (1990). Merger and Free Riders in Spatial Markets. FTC Working Papers. Washington DC, Bureau of Economics FTC. 
Reitzes, J. D. and D. T. Levy (1995). "Price Discrimination and Mergers." The Canadian Journal of Economics 28(2): 427-436.

Salant, S.W. and S. Switzer, and R.J. Reynolds (1983). "Losses from Horizontal Merger: The Effects of an Exogenous Change in Industry Structure on Cournot-Nash Equilibrium" The Quarterly Journal of Economics 98(2): 185-199

Salop, S. C (1987). "Symposium on Mergers and Antitrust" The Journal of Economic Perspectives 1(2): 3-12.

Salop, S. C (1979). " Monopolistic Competition with Outside Goods." The Bell Journal of Economics 10(1): 141-156.

Salop, S. C (1995). "Efficiencies in Dynamic Merger Analysis-Statement of Steven C. Salop." Federal Trade Commision-Hearings On Global And Innovation-Based Competition Nov. 2.

Shapiro, C. (1996). "Mergers with Differentiated Products." Antitrust 10(2): 23-30.

Szidarovszky, F. and S. Yakowitz (1982). "Contributions to Cournot oligopoly theory." Journal of Economic Theory 28(1): 51-70.

Vives, X (1986). "Rationing Rules and Bertrand-Edgeworth Equilibrium in Large Markets." Economics Letters 21(2): 113-116.

Weinberg, M (2008). "The price effects of horizontal mergers. " Journal of Competition Law \& Economics 4(2): 433-447.

Werden, G.J. (1996). "A Robust Test for Consumer Welfare Enhancing Mergers Among Sellers of Differentiated Products." The Journal of Industrial Economics 44(4): 409413.

Werden, G. J, L. M. Froeb and S. Tschantz (2005). " The effects of Merger Efficiencies on Consumers of Differentiated Products." European Competition Journal 1:245264.

Williamson, O.E (1968). "Economies as an Antitrust Defense: The Welfare Tradeoffs." The American Economic Review 58(1): 18-36.

Yde, P. L. and M. G. Vita (1996). "Merger efficiencies: Reconsidering the 'passing on' requirement." Antitrust Law Journal 64(3): 735-747.

Yde, P. L. and M. G. Vita (2006). "Merger Efficiencies: The Passing-On Fallacy." Antitrust 20(3): 59-65. 